\title{
The role of Ndc80 and Ska complexes in maturation of kinetochore-microtubule attachments
}

\author{
Pawel Lukasz Janczyk \\ Gdynia, Poland \\ B.S. Biotechnology, University of Gdansk, 2007 \\ B.S. Chemistry, University of Gdansk, 2009 \\ M.S. Biotechnology University of Gdansk, 2009

\begin{abstract}
A Dissertation presented to the Graduate Faculty of the University of Virginia in
\end{abstract} \\ Candidacy for the Degree of Doctor of Philosophy \\ Interdisciplinary Program in Biophysics \\ University of Virginia \\ April 2016
}




\section{Table of Contents}

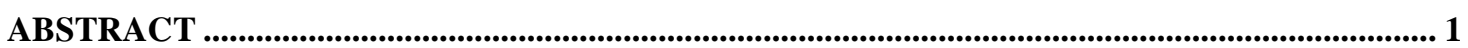

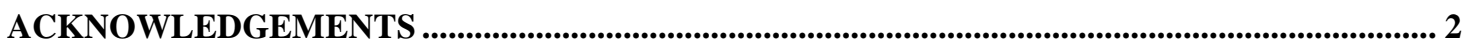

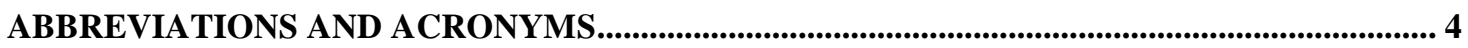

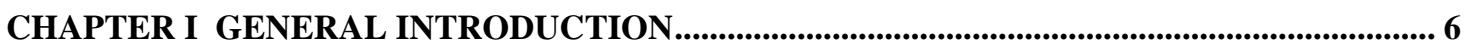

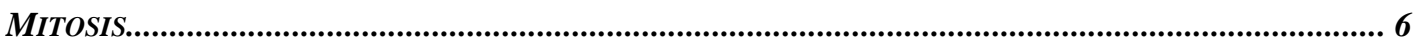

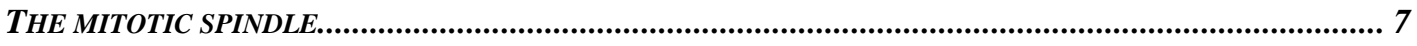

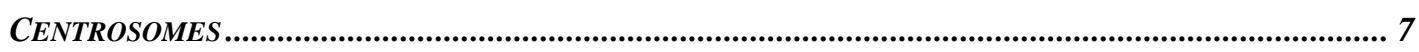

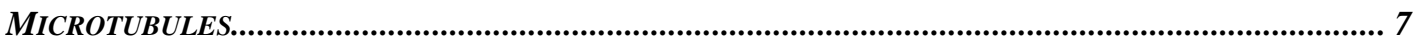

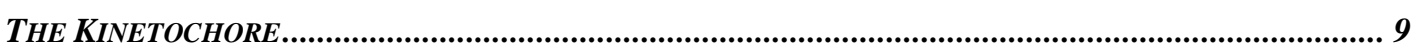

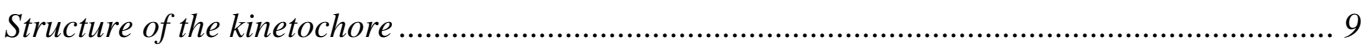

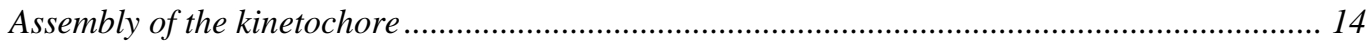

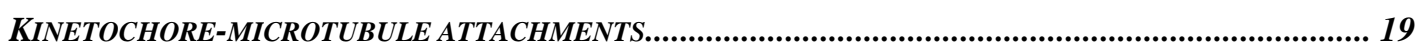

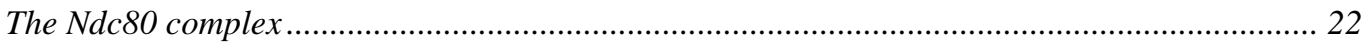

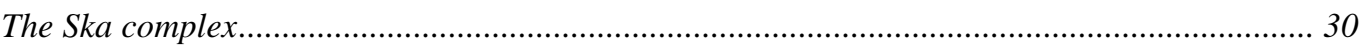

MATURATION OF KINETOCHORE-MICROTUBULE ATTACHMENTS ……............................................... 37

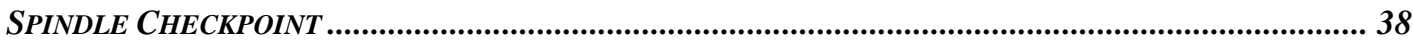

Mechanism of Spindle Checkpoint signaling........................................................................ 39

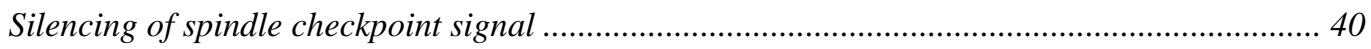

RECOGNITION OF THE MATURE KINETOCHORE-MICROTUBULE ATTACHMENTS BY THE SPINDLE

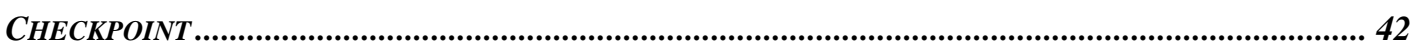

CHAPTER II THE UNSTRUCTURED TAIL OF NDC80 RECRUITS SKA TO

KINETOCHORES TO FACILITATE EXIT FROM MITOSIS ......................................................... 45

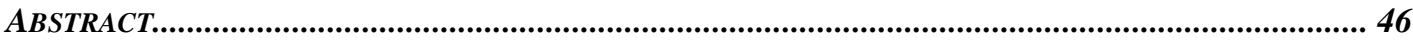

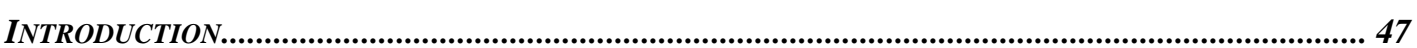

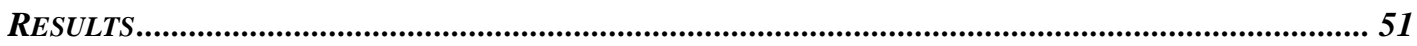

Positive charge in the $\mathrm{N}$-terminal region of the $\mathrm{Ndc80}$ tail is critical for chromosome congression in vivo.

The charge in the C-terminal region of the tail is required to silence the spindle checkpoint..... 52

$\mathrm{Ndc} 80^{+4 C T}$ arrests cells in metaphase due to both SAC and Aurora B activation.....

C-terminal mutant of the Ndc80 tail retains high affinity to microtubules but lacks ability to

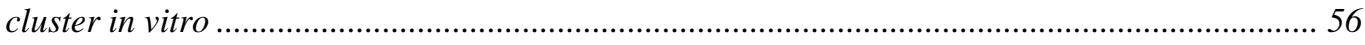

C-terminal region of $\mathrm{Ndc80}$ tail is required for Ska3 recruitment to the kinetochore .................. 57

Ska3 recruitment to the kinetochore is dependent on Aurora B phosphorylation of Ndc80 tail. . 58

Ska complex forms $V$-shaped structures on microtubules in presence of $N d c 80 \ldots \ldots \ldots . . . . . . . . . . . . . .60$

DISCUSSION.

Insight into "end-on" attachment by the kinetochore and depolymerization-coupled movement 61 Insight into the final stages of "end-on" attachments and silencing of the spindle checkpoint... 63

MATERIAL AND METHODS 65

FIGURES 70 


\section{CHAPTER III THE HUMAN SKA COMPLEX DRIVES THE METAPHASE-ANAPHASE CELL CYCLE TRANSITION BY RECRUITING PROTEIN PHOSPHATASE 1 TO}

KINETOCHORES

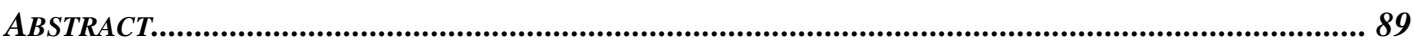

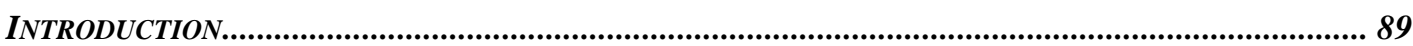

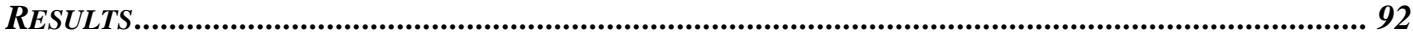

The Ska complex is required for kinetochore accumulation of PP 1 ............................................. 92

The Ska complex directly interacts with PP1 through Ska3 subunit. ........................................ 93

Interaction with PP1 is facilitated by multiple sites on Ska3 ............................................... 94

Skal CTD contributes to Ska-PP1 interaction.......................................................................... 95

The Skal CTD is required for PPI kinetochore targeting and Knll dephosphorylation............ 96

Ska-mediated kinetochore recruitment of PP1 promotes anaphase onset................................... 97

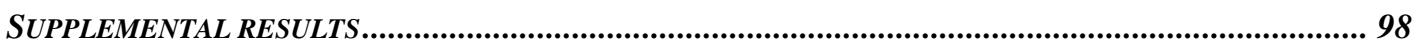

B55 subunits of the PP2A complex interact with Ska3..................................................... 98

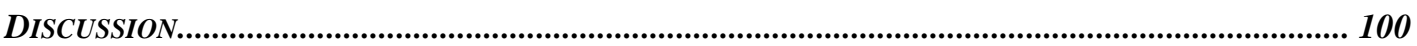

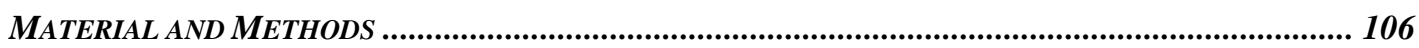

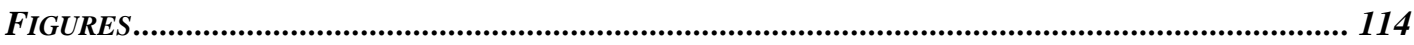

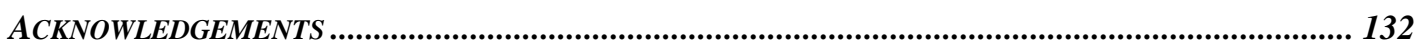

CHAPTER IV GENERAL DISCUSSION AND FUTURE PERSPECTIVES .............................. 133

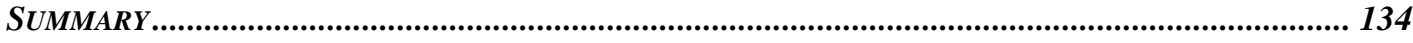

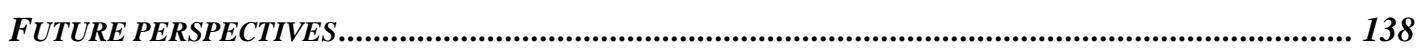

Identification of the components of the $V$-shaped structures.................................................. 138

Characterization of the requirements for the assembly of the Ndc80-Ska supercomplex and subsequent recruitment of PP 1 ............................................................................................. 139

Ndc80-dependent Ska recruitment in the absence of microtubules ....................................... 140

NSP as a microtubule coupler required for chromosomal movements ...................................... 141

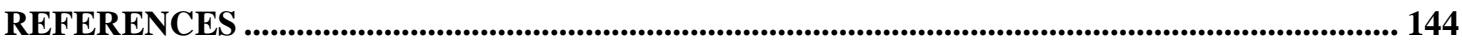




\section{Abstract}

In eukaryotes, segregation of genetic material is a complex and highly regulated process. From the condensation of the chromosomes, through the kinetochore assembly, formation of microtubule attachments, congression and segregation, until the completion of cytokinesis, each step has to be precisely coordinated to allow for faithful distribution of the DNA to daughter cells. Although our understanding of those processes significantly improved over recent years, one important mechanism is yet to be properly described: maturation of kinetochore-microtubule attachments. Several kinetochore components have been identified as essential for formation and maintenance of end-on microtubule attachments. However, it is still unclear how kinetochore-microtubule attachments mature to allow for mitosis completion. The Ndc80 complex was identified as an essential microtubule binder that allows for graded stability of the attachments by a phosphorylation-dependent regulation mechanism. Ten years ago a novel complex, Ska, was discovered as a component of the mitotic spindle and implicated in the attachment maintenance and chromosomal segregation. While some studies indicate that Ska serves as the coupler for depolymerization driven movements, other suggest it is crucial for spindle checkpoint silencing. In this dissertation I investigate the dependencies between Ndc80, Ska and PP1 phosphatase, their roles in the maturation and recognition of the microtubule attachments. I present data indicating that Ska is recruited to the kinetochores through the $\mathrm{N}$-terminal tail of the $\mathrm{Ndc} 80$ protein and that the resulting complex forms characteristic structures on the surface of the microtubules. Moreover, the Ska complex binds to and recruits PP1, one of the major mitotic phosphatases required for dephosphorylation of mitotic substrates and, as a result, mitotic exit. Overall, the data presented here strongly suggest that Ndc80-Ska-PP1 network allows kinetochores to mature end-on attachments and couple the binding of microtubules to spindle checkpoint silencing. 


\section{Acknowledgements}

I have spent multiple years at UVA and there are so many people I would like to thank. Without them I would not be able to get to this point of my life.

First and foremost, I would like to thank my mentor, Dr. Todd Stukenberg, for years of continuous support and advice. Despite the numerous challenges my projects presented, you always had your door open, ready to help when needed. Also I thank you for the independence you gave me and your patience, whenever I bothered you with some simple or obvious questions.

I would also like to thank my graduate committee members, Drs. Dan Burke, Robert Nakamoto, David Brautigan and Zygmunt Derewenda. Especially, I would like to thank Dr. Zygmunt Derewenda who initially recruited me to be a part of the master student exchange program and introduced me to the vast scientific community here at UVa. I cannot underestimate how much it influenced my life. Thank you Dr. Nakamoto for being the best graduate program administrator one could wish for, and for all the advice you gave me. I also thank Dr. David Brautigan. Although I asked you to join the committee only a year ago, your vast knowledge was especially helpful. Also I thank you for all the reagents you provided.

Dr. Dan Burke - over the years of my $\mathrm{PhD}$ you gave me so much advice that was both critical and very much practical. It is impossible to underestimate your influence on the way I think about science. I will never forget your comments, and I hope you know that the lab meetings are not the same without you. However, I am sure some traditions will continue for the years to come (the classic "let my people go").

I thank all previous and current lab members: Sara Rosasco-Nitcher, Anne Knowlton, Brian Fuller, Stephanie Miller, Cortney Kestner, Sarah Louie, John Tooley, Ewa 
Niedziałkowska, Eliza Żyłkiewicz, Dan Matson, Budhaditya Banerjee, Arkadi Manukyan, Limin Liu and Prasad Trivedi. Special thanks to Prasad who took his time to critically read this dissertation (parts of it at least) and for all the invaluable conversations we had. Your knowledge and your understanding of the mitosis are just astonishing.

Obviously, years in Charlottesville were not only spent on studying and research, albeit it sometimes felt that way. As I met a lot of great people here, it would be impossible to mention all of them. However, one person easily stands out from that crowd: Janusz Jurand Pętkowski (or JJ for friends). Although we were roommates for only two short years I will never forget all the conversations we had, as well as our "cygaro nights" with Sci-fi movies. Special thanks for introducing me to homebrewing!!

A very special thanks to my lovely girlfriend, Kasia Skorupka, for being the best girlfriend one could ever imagine. You are the best thing that could have happened to me. Thank you for being the person you are, always close when in need. Special thanks for your patience and understanding, especially in recent months when I was so occupied with writing this dissertation.

Lastly, I thank my parents and my brother for their continuous support over the years of my studies, and my life in general. The whole length of this dissertation would not be sufficient to list all the things I am thankful for! 


\section{Abbreviations and acronyms}

$\AA$

APC/C $\quad$ Anaphase Promoting Complex/Cyclosome

ATP

bp

BRB80

BSA

CCAN

$\operatorname{Cdc} 20$

$\mathrm{Cdk}$

CENP

CHD

CSF

CTD

DNA

DTT

EM

FBS

FL

GDP

GST

GTP

$\mathrm{kb}$

K-fiber

KMN

Ångström

Adenosine triphosphate

base pair

Brinkley Reassembly Buffer $\underline{80}$

Bovine $\underline{\text { Serum }}$ Albumin

Constitutive Centromere Asssociated Network

Cell Division Cycle 20

Cyclin-Dependent $\underline{\text { Kinase }}$

Centromeric protein

Calponin Homology Domain

Cytostatic Factor

C-terminal Domain

Deoxyribonucleic a acid

Dithiothreitol

Electron Microscopy

Fetal Bovine Serum

Full-length

Guanosine diphosphate

Glutathione $\underline{\text { S-transferase }}$

Guanosine triphosphate

kilobase

Kinetochore-associated microtubule fiber

Knl1/Mis12/ㅁdc80 complex 
KT

MCAK

MCC

MT

MTBD

MTOC

MST

MW

PBS

PCM

PCR

PLA

PP1

RNA

RNAi

SAC

SEC

SDS-PAGE

siRNA

Ska

SKAP

TBS

WT
Kinetochore

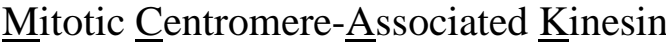

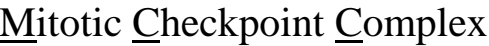

Microtubule

Microtubule Binding Domain

$\underline{\text { Microtubule Organizing Center }}$

Microscale Thermophoresis

Molecular Weight

Phosphate-Buffered $\underline{\text { Saline }}$

Pericentriolar matter

Polymerase $\underline{\text { Chain }}$ Reaction

Proximity Ligation Asssay

Protein Phosphatase $\underline{1}$

Ribonucleic acid

RNA interference

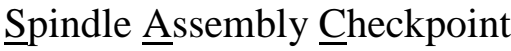

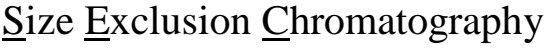

Sodium Dodecyl Sulphate-Polyacrylamide Gel Electrophoresis small interfering $\underline{\mathrm{RNA}}$

Spindle and kinetochore associated complex

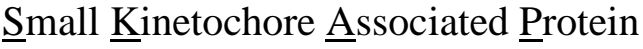

$\underline{\text { Tris-B }}$ uffered $\underline{\text { Saline }}$

wild-type 


\section{Chapter I.}

\section{General introduction}

\section{$\underline{\text { Mitosis }}$}

To ensure the propagation of the genetic material, each cell undergoes a process known as a cell division. In prokaryotes, it usually occurs by binary fission. This process requires replication of DNA and cytokinesis. The physical attachment of the replicated DNA to the opposite region of the cell membrane ensures separation during cell division. Eukaryotic genetic material is more difficult to manipulate, however. Eukaryotic DNA requires very precise separation to ensure that the replicated DNA is properly segregated to the daughter cells. In somatic cells of eukaryotes the most common form of cell division is known as mitosis. It consists of multiple stages: prophase, prometaphase, metaphase, anaphase and telophase. When entering mitosis, DNA condenses forming chromosomes, and the nucleolus is dissolved. In the next step, prometaphase, nuclear membrane breaks apart and chromosomes assemble multiprotein kinetochore structure that makes direct attachments with microtubules, creating a mitotic spindle. Metaphase is defined by the congression of chromosomes into the metaphase plate. Upon alignment of the last chromosome to the metaphase plate, anaphase is initiated. Cohesion between sister chromatids is lost allowing their physical separation in anaphase. As the rapidly depolymerizing microtubules remain attached to the kinetochore, sister chromatids are pulled apart to the opposite ends of the cell. After all chromatids are separated, the nuclear membrane is reassembled and DNA is decondensed during telophase. Physical separation of the genetic material is concluded by the cytokinetic furrow that contracts the cell membrane between the separated chromatids and creates two genetically identical daughter cells. 


\section{$\underline{\text { The mitotic spindle }}$}

The mitotic spindle is a specialized, dynamic structure that consists of three main components: chromosomes, spindle microtubules and a pair of centrosomes. It ensures a proper and symmetrical separation of the genetic material to the daughter cells. Below I discuss the major structures and controlling mechanisms involved in formation, maintenance and functioning of the mitotic spindle.

\section{Centrosomes}

The main function of centrosomes is to serve as a microtubule organizing center (MTOC). They also play a role in adhesion and polarity during interphase. Centrosomes contain two sister centrioles surrounded by pericentriolar matter (PCM) [1]. The PCM contains proteins such as pericentrin, ninein and $\gamma$-tubulin that are responsible for microtubule nucleation and anchoring [2]. The two centrioles are situated perpendicular to one another and are comprised of proteins such as centrin, canexin and tektin [3]. Centrosomes duplicated in S-phase separate from each other at the beginning of mitosis and move to the opposite ends of the cell, nucleating, organizing and anchoring spindle microtubules. In a process called "search and capture" those microtubules probe the cytoplasm to find chromosomes they can attach to [3]. Although "search and capture" is predominant in most cell types, another mechanism called "self-organization" exists. It is observed in acentrosomal systems where microtubules are nucleated from chromatin and their further organization to form mitotic spindle is driven by motors and microtubule associated proteins (reviewed in [4]).

\section{$\underline{\text { Microtubules }}$}

Spindle poles and chromosomes are connected to each other through long protein polymers called microtubules. The building block of the microtubule polymer is a protein heterodimer that consists of closely related $55 \mathrm{kDa}$ polypeptides of $\alpha$ - and $\beta$-tubulin. 
Multiple tubulin subunits line up end-to-end to form protofilaments that associate laterally to form a single cylindrical tube, the microtubule. Typically, in vivo microtubules are formed by thirteen protofilaments, making tubes of $25 \mathrm{~nm}$ diameter; in vitro however, the number of protofilaments within one microtubule can vary from eight to twenty [5-8].

As the tubulin heterodimer is polarized, so are microtubules. The end with $\beta$ tubulin subunit is called the plus (+) end, while the minus (-) end contains $\alpha$-tubulin. The polarity of the microtubule is reflected in the polymerization rates. While polymerization happens on both ends, the (+) end elongates significantly faster than the (-) end. Because tubulin has GTPase activity, the GTP bound to $\beta$-tubulin (but not that bound to $\alpha$-tubulin) is hydrolyzed after the addition of tubulin dimer. Usually hydrolysis occurs with a short delay, resulting in formation of a cap of GTP-bound $\beta$-tubulin at the growing microtubule (+) end. Energy of GTP hydrolysis is not immediately released by the microtubule, but is conserved within the microtubule lattice. It is largely owed to the GTP "cap" that stabilizes the straight conformation of the microtubule (+) end. GTP hydrolysis weakens interaction between adjacent tubulin subunits. The polymerization/depolymerization state of microtubule is directly related to the rate of tubulin dimer addition versus the rate of the GTP hydrolysis. As the rate of polymerization slows, the GTP cap at the plus end of the microtubule undergoes hydrolysis to GDP. Once the GTP cap is lost, a rapid depolymerization occurs and the energy stored laterally within the microtubule is released. During the depolymerization GDP-bound tubulin subunits peel off the microtubules creating curved filaments at the plus ends (e.g. [9]). This switch from growth to shrinkage is also known as a "catastrophe" event, while the switch from shrinkage to growth is called "rescue". Microtubules are dynamically unstable and undergo cycles of growing and shrinking. This behavior is widely referred to as "dynamic instability" and can be observed both in vitro and in vivo [10,11]. Microtubule dynamics 
in vitro are influenced by factors such as: tubulin concentration, temperature, abundance of free GTP and viscosity of the solution $[10,12]$. In vivo, that list additionally includes multitude of regulatory proteins that are believed to account for the differences in microtules behavior and dynamics $[13,14]$.

\section{$\underline{\text { The Kinetochore }}$}

The kinetochore is a multiprotein structure that assembles on the centromeric region of chromosomes during mitosis. Its basic function is linking the chromosomes to the mitotic spindle. This, however, is a very general description, as the kinetochore does not only serve as a linkage, but also a regulator of multiple processes, including microtubule dynamics, detection and correction of improper attachments, as well as assembling and maintaining the Spindle Assembly Checkpoint (SAC, also referred to as Spindle Checkpoint) until all chromosomes have formed proper attachments and aligned at the metaphase plate. For a long time only few proteins have been identified as kinetochore components, including CENP-A, CENP-B and CENP-C [15,16]. Multiple kinetochore components were identified since the 2000's, and around 100 proteins have been discovered and characterized in vertebrate kinetochores up until now (reviewed in [17-20]). Below I focus on the kinetochore architecture and describe some of the kinetochore components and their roles in chromosome congression and segregation.

\section{Structure of the kinetochore}

Studies that utilized electron microscopy (EM) on vertebrate cells following fixation and staining [21-24] or high-pressure freezing [25] shed light on the tri-laminar nature of the kinetochore structure. Assembled on top of the centromere, the vertebrate kinetochore contains a pair of electron dense layers (referred to as inner and outer kinetochore plates, each $\sim 40 \mathrm{~nm}$ thick), separated by an electron-opaque middle layer ( 27 $\mathrm{nm}$ thick) (Figure 1.1). EM studies also revealed a region, named fibrous corona, 
emanating from the outer plate, clearly visible only in the absence of microtubules [26]. The chromatin located between two sister kinetochores is called the inner centromere (reviewed in [17,27]). So far three types of centromeres were characterized: point centromere, regional centromere [28] and holocentromere that is distributed along whole chromosome [29]. While point centromere observed in budding yeast attaches a single microtubule polymer, centromeres of all higher eukaryotes that were studied so far bind with multiple spindle microtubules. For example each human kinetochore is associated with 15-20 microtubules [30]. Recent studies on holocentromeres in C. elegans suggest that they are polycentromeres assembled by point centromeres containing cenH3 (CENPA) nucleosomes dispersed along the chromatid [31] .

The inner plate of the kinetochore consists of proteins assembled on the centromeric region of the chromatin and is predominantly composed of centromere associated proteins (CENPs) that remain centromere-associated throughout the cell cycle. One of the epigenetic markers of the centromere is the histone H3 variant CENP-A [16,32-35] that is required for assembly of all kinetochore components [36]. CENP-A interacts directly with centromeric chromatin as well as other kinetochore proteins such as CENP-C and CENP-N [37]. Purification of CENP-A nucleosomes from human cells revealed a network of proteins constitutively associated at centromeres. This network of sixteen proteins is called Constitutive Centromere Associated Network (CCAN). CCAN is divided into several subgroups: the CENP-C, CENP-T/W/S/X, CENP-H/I/K/M, CENP-L/N, and CENP-O/P/Q/R/U groups (reviewed in [38]). CENP-C and CENP-T/W were shown to be mediators between centromeric chromatin and outer kinetochore components [39-41].

The outer plate of the kinetochore is assembled on the inner kinetochore after the nuclear envelope breakdown and it persists throughout mitosis until late anaphase. The 
main functions of the outer kinetochore include formation of stable microtubule attachments, chromosome congression and signaling the spindle checkpoint. The primary point for the end-on microtubule attachments is formed by the KMN network [42]. The KMN network consists of Knl1, Mis12 complex (Mis12, Nnf1, Dsn1 and Ns11) and Ndc80 complex (Ndc80, Nuf2, Spc24 and Spc25). The Ndc80 complex will be described in detail later. Zwint, a protein that directly interacts with Knl1, could be considered as another member of the KMN network [43-45].

Fibrous corona can be best observed on the EM images of unattached kinetochores in cells treated with drugs preventing microtubule polymerization. It is a region most distant from the centromere and the amount of protein material is dependent on the status of microtubule attachments. When the chromosome is aligned in the metaphase plate, multiple components are stripped from the fibrous corona via minus end directed movements mediated by dynein complex [46]. Two classes of proteins can be distinguished within the fibrous corona. In the first class are proteins that are involved in creating initial, lateral attachments with microtubules. Among those are CENP-E and dynein. As the lateral kinetochore-microtubule interactions are transient in nature and are not sufficient for coupling depolymerization-driven chromosomal movement $[47,48]$, stable bipolar end-on attachments must be formed to allow for efficient chromosome segregation. The second class of the proteins residing within the fibrous corona are spindle checkpoint proteins. Among them are Mad1, Mad2, Mad3, Bub1, BubR1 (Bub3) and in vertebrates the RZZ complex (Rod, Zwilch and ZW10). Spindle checkpoint is discussed in detail later. 


\section{Figure 1.1 Schematic representation of kinetochore structure.}

A plate-like architecture of the kinetochore as observed in EM studies. Representative slice from an EM tomogram of the microtubule plus-ends embedded in the outer kinetochore (top image) and a thin cross-sectional view of a cold-stable kinetochore fibers (bottom image).

Reproduced from [49] with permission (License: 3835241507803 ) 
Figure 1.1

Kinetochore-Attached Plus Ends

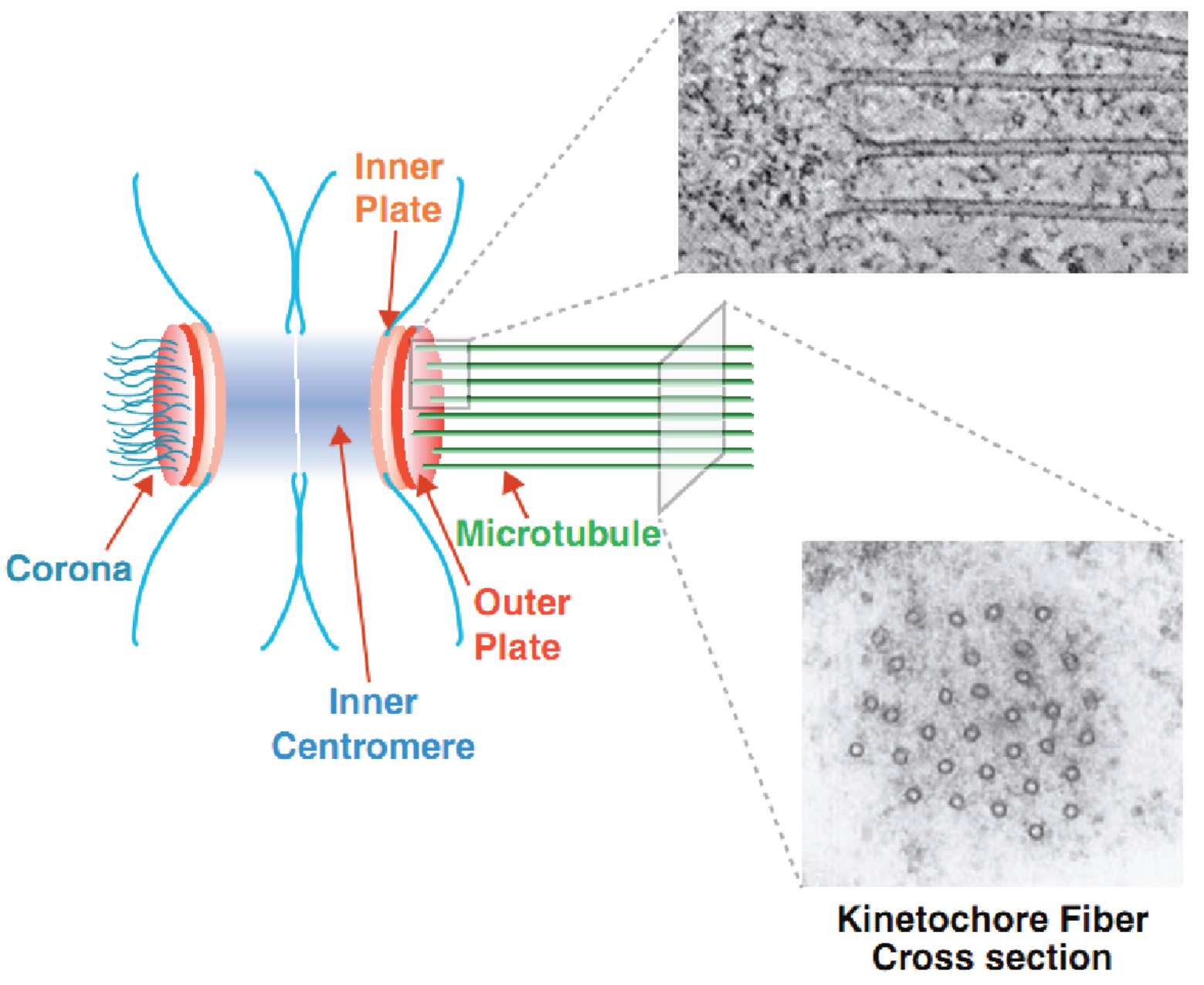




\section{Assembly of the kinetochore}

To allow for faithful chromosome segregation to daughter cells, assembly of segregation machinery must be carefully regulated, both spatially and temporally. The kinetochore is built on centromeric chromatin that is spatially restricted to a single region (monocentromere) in most eukaryotes. The size and the nature of the centromeric DNA varies across the species. In the budding yeast Saccharomyces cerevisiae the centromeric region is defined by a $\sim 125$ bp point centromere localized within a $\sim 200$ bp nucleaseresistant region [50-52]. The majority of eukaryotes have much larger centromeres, however, ranging from 40-100 kb in fission yeast Schizosaccharomyces pombe, up to 0.35 megabases in some animal and plant species [53]. Although some species need specific DNA sequences to define the centromere, most organisms do not have this requirement. The sequence-independent nature of centromeres suggests that it is defined and maintained epigenetically. One of the important proteins in the centromere is histone $\mathrm{H} 3$ variant CENP-A (Cnp1 in Sc. pombe, Cse4 in S. cerevisiae, Cid in Drosophlia and HCP3 in C. elegans). Because CENP-A nucleosomes are localized specifically to centromeric chromatin $[32,34]$ and are deposited only at sites with preexisting CENP-A, it is thought to act as a centromere epigenetic marker [54].

The connection between DNA and microtubules is believed to be formed by two major "branches". The first branch is built by CENP-C, which directly interacts with the CENP-A nucleosomes $[37,55]$. CENP-C was shown to be involved in the recruitment of kinetochore proteins, such as CENP-E, Mad2 and the KMN network through the Mis12 complex (also known as the MIND complex) [39-41,56-58]. Specifically, the Nterminus of CENP-C directly binds with the Nnf1 subunit of the Mis12 complex. Another Mis12 complex subunit, Nsl1, interacts with Knl1 and the C-terminal region of the Ndc80 complex, thereby recruiting the whole KMN network to the kinetochore. The 
KMN network itself serves as a platform for recruitment of additional microtubule binding and spindle checkpoint proteins [59].

The second branch involves the CENP-T/W/S/X complex that was shown to be structurally similar to nucleosomal histones [60]. The elongated N-terminal region of CENP-T directly interacts with the Ndc80 complex $[39,60]$. To generate proper microtubule attachments, both CENP-C and CENP-T branches are essential in vertebrate cells. Furthermore, N-terminal regions of CENP-C and CENP-T were shown to be sufficient to form a kinetochore-like structure in an ectopic location lacking CENP-A nucleosomes $[39,61,62]$.

Previous studies have demonstrated variations in the dependencies and physical interactions between kinetochore components throughout evolution $[17,42,44]$. Studies done in organisms such as budding yeast, fission yeast and chicken suggest that, despite a wide range of microtubules binding to each kinetochore, the overall number of kinetochore proteins present at the microtubule binding site is usually similar between species [63-65]. It suggests a model in which the kinetochores are formed by multiple microtubule binding "units", which are basically repetitions of a single microtubule binding site similar to one employed by budding yeast. Dam1 protein seems to be an exception, as in budding yeast there are 16 Dam1 molecules per microtubule, while in fission yeast, where Dam1 is not essential, there is only one Dam1 per microtubule $[64,66,67]$. Based on recent EM, cell biology and modeling studies [68-72], a novel model was proposed, suggesting a different arrangement of microtubule binding components. Instead of a repetition of microtubule-binding sites the kinetochore could form a lawn of individual microtubule-binding proteins, where the possibility of the interaction with microtubule is determined only by the distance to the nearest microtubule (Figure 1.2) [69]. This model simplifies microtubule capture and could allow for 
cooperativity between different kinetochore components. It has also been speculated that the lawn could be made of a flexible, velcro-like layer allowing for structural rearrangements upon microtubule binding. It would influence not only molecular states of the microtubule-bound proteins but also permit changes in the properties of molecules surrounding a microtubule attachment site [73]. 


\section{Figure 1.2 Models for the microtubule binding}

Left - Repetitive site model of the kinetochore. Circles indicate individual microtubule binding sites able to attach a single microtubule. Red fibers indicate Ndc80 molecules responsible for microtubule attachments. The model assumes no cross-talk between individual microtubule binding sites. This model cannot explain the observed relationship between phosphorylation status of microtubule binding proteins and the number of kinetochore-microtubule attachments.

Right - Molecular lawn model of the kinetochore. No individual microtubule binding regions are specified, attachment occurs at random positions and binding of another microtubule can modulate the binding efficiency in proximal regions. The exact positions of attached microtubules changes with time, but the total number of microtubules bound to the kinetochore remains in a steady state. Recent modeling studies favor the molecular lawn model as it properly simulates phospho-regulation of the kinetochore-microtubule attachments observed in vivo.

Adapted from [69]. 
Figure 1.2

Repetitive site model

Molecular lawn model
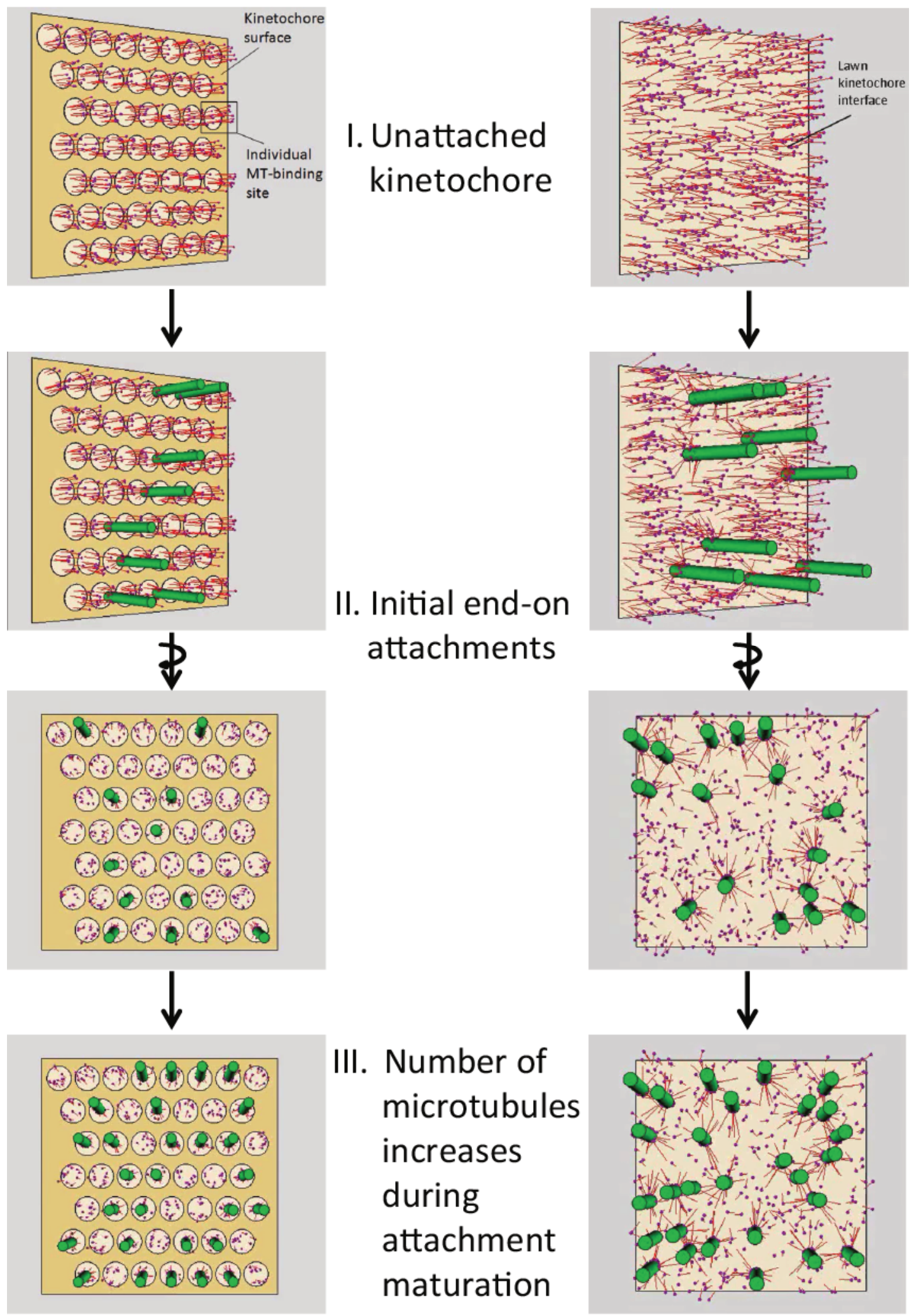

II. Initial end-on attachments
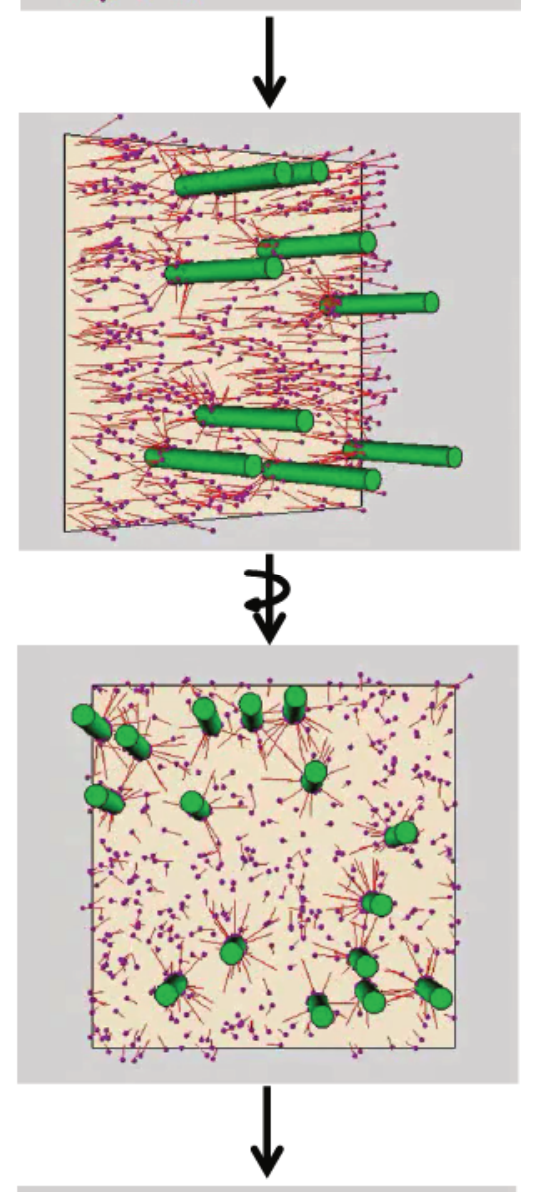

III. Number of microtubules increases during attachment maturation

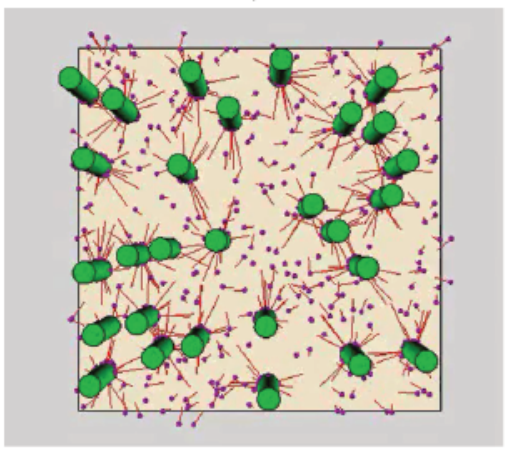




\section{Kinetochore-microtubule attachments}

The most important role of the kinetochore in mitosis is to form stable attachments with the mitotic spindle that allow the chromosomes to segregate in anaphase. Through the "search and capture" mechanism, microtubules initially bind laterally to the outer kinetochore (Figure 1.3). Those attachments allow the kinetochores to undergo movements, mediated by motor proteins such as dynein/dynactin and CENPE. The movement of chromosomes through motor proteins requires an energy stroke generated by ATP hydrolysis [74-77]. Studies suggested that the congression of chromosomes does not explicitly require stable end-on attachments and that either lateral or transient end-on attachments are sufficient [78,79]. Those, however, do not allow for stable maintenance of the chromosomes at the metaphase plate [47,48]. Although it is still unclear how the transition from lateral to end-on attachments occurs, it has been suggested that it is a gradual, multistep process [80]. Unlike the ATP-stroke utilized by motor proteins to move laterally attached chromosomes, the force required to move chromosomes with end-on attachments is generated by microtubule plus-end depolymerization [81]. Attachment of microtubule by the kinetochore is facilitated by microtubule-binding proteins within the outer kinetochore. Below I describe two protein complexes implicated in end-on attachment formation and maintenance. 
Figure 1.3 Kinetochore-microtubule interactions in prometaphase and metaphase.

(a) Kinetochores are initially attached to the lateral surface of microtubules extending from a single spindle pole following nuclear envelope breakdown (in metazoan cells) or once assembly of the kinetochore is complete (in budding yeast)

(b) Kinetochores are transported along the microtubules toward the spindle pole. While transported, one or both of the sister kinetochores attaches to the lateral surface of the microtubule during the chromosome movements.

(c) As kinetochores approach spindle poles, both sister kinetochores attach to microtubules extending from the same (upper panel) or opposite (lower panel) spindle poles.

(d) Bi-orientation of sister kinetochores. The number of microtubules forming end-on attachments with single kinetochore increases in metazoan cells, whereas only a single microtubule attaches to each kinetochore in budding yeast (shown here for simplicity). Reproduced from [82] with permission (license: 3842770136435 ) 
Figure 1.3
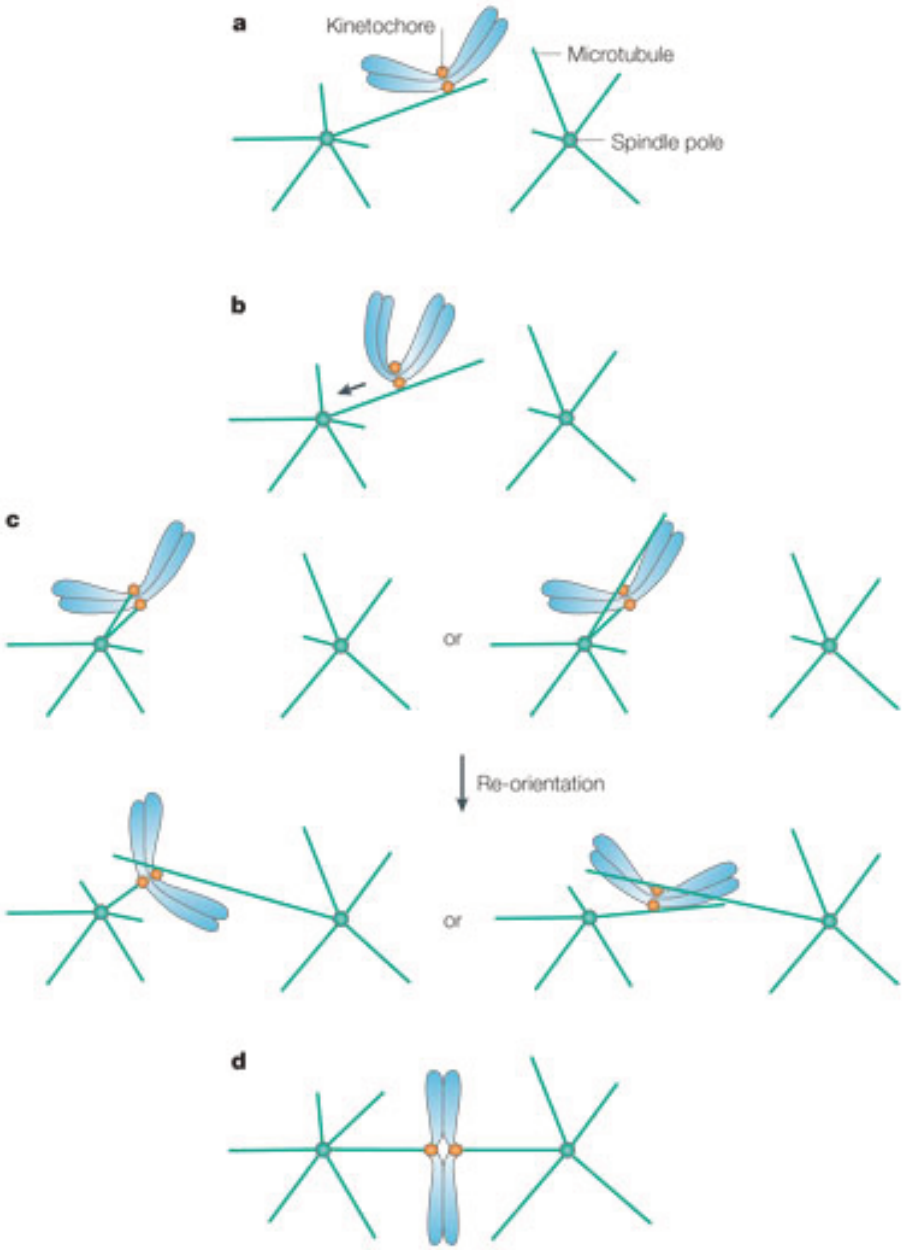

Copyright 02005 Nature Publishing Grous Nature Reviews | Molecular Cell Biology 


\section{The Ndc80 complex}

A number of kinetochore proteins have been shown to bind directly to microtubules, e.g. CENP-E, CENP-F, dynein, MCAK, Knl1, the Ndc80 complex and Ska complex. Of those, the nuclear division cycle 80 complex (Ndc80) appears to be essential for the formation of stable kinetochore-microtubule interactions in a number of organisms [83-87]. This complex is responsible for performing multiple functions that are critical for the outer kinetochore. Those include: robust end-on attachment to $(+)$ ends of microtubules, linking kinetochores with the spindle poles; force generation by microtubule plus-end depolymerization; phosphorylation-dependent correction of kinetochore-microtubule attachment errors; regulation of spindle checkpoint $[59,81,88,89]$.

The Ndc80 complex is comprised of four proteins, Ndc80 (also known as Hec1), Nuf2, Spc24 and Spc25, in a 1:1:1:1 stoichiometry [42,86,90-93]. All four members interact with each other with high affinity, forming a tight complex both in vitro and in vivo $[83,87]$. Depletion of any of the complex subunits results in drastic abnormalities in chromosome alignment and mitosis progression. Extensive structural studies of the Ndc80 complex demonstrated that it forms a $57 \mathrm{~nm}$ long rod-shape structure with two globular heads at each end, linked through the extended coiled-coil (Figure 1.4.A) [9093]. One end the Spc24/Spc25 dimer forms a receptor that tethers the complex to the kinetochore through Mis12 and CENP-T interactions [39,40,44,94-96]. The other end, consisting of Ndc80 and Nuf2 dual calponin homology domains (CHDs), directly binds to microtubule polymers $[93,97]$. The long coiled-coil region between Ndc80/Nuf2 and Spc24/Spc25 is disrupted by a loop region that results in a kink (also called a "hinge"), positioned $\sim 16 \mathrm{~nm}$ away from the globular microtubule binding end. It was previously suggested that the kink allows for a certain level of flexibility of the complex $[92,98,99]$. In fission yeast, the Ndc80 loop interacts with Dis1/TOG microtubule-associated protein 
[100], while in budding yeast it binds with Dam1 complex [101]. In humans, the kink binds a DNA replication licensing factor Cdt1 that regulates the bending of the coiledcoil [102]. One study suggested the role of the "hinge" region in recruitment of the Ska complex [103], however a different group did not observe a similar phenotype in the Ndc80 loop mutants [88].

The sequence of the Ndc80 N-terminal tail is highly divergent among species. In vivo studies also show phenotype diversity: the Ndc80 tail is not essential in budding yeast or C. elegans, however it is crucial for viability in human cells [49,104-106]. The human Ndc80 complex associates with microtubules in a cooperative fashion through Ndc80 CHD and the 80 amino-acid long unstructured N-terminal tail of Ndc80 protein. Both of those regions are required for creating proper microtubule attachments and it was shown that each contributes to the binding affinity $[42,92,93,105,107,108]$. Additionally, removal of the negatively-charged C-terminal tail of tubulin (E-hook) or introduction of charge disrupting mutations in CHDs results in a dramatic reduction of binding affinity between microtubules and Ndc80. It suggests a model of binding based on ionic interactions between the positively charged residues of $\mathrm{Ndc80}$ and the negatively charged residues on tubulin subunits $[93,105]$. Electron microscopy studies using recombinant $C$. elegans $\mathrm{Ndc} 80 / \mathrm{Nuf} 2^{\mathrm{HIM}-10}$ and microtubules grown from centrosomes attached to EM grids indicated that the $\mathrm{CeNdc} 80 / \mathrm{Nuf} 2 \mathrm{CHD}$ domains bind at $\sim 60^{\circ}$ angle between adjacent tubulin monomers, pointing towards centrosomes (i.e. (-) end). The C-terminal end of the complex, corresponding to Spc24/Spc25 subunits, extends away from the microtubule lattice, at an angle $\sim 20-60^{\circ}$, pointing towards microtubule plus ends [99]. Given the length of the complex $(\sim 57 \mathrm{~nm})$ and the flexibility of the kink region, this binding geometry could allow the Ndc80 complex to bind to the kinetochore through the Spc24/Spc25 end and leave enough room for polymerization and depolymerization of the microtubule plus end. $C e$ Ndc80/Nuf2 molecules attached to microtubules were visualized 
using helical reconstruction of cryo-EM micrographs, forming asymmetrical interactions, with alternating patterns of strong and weak densities positioned along the protofilaments (clusters). $\mathrm{CeNdc80/Nuf2}$ appears to preferentially bind to $\beta$-tubulin $[99,109]$.

In subsequent studies Alushin et al., 2010 [108] obtained an $8.6 \AA$ resolution electron density map of an engineered human $\mathrm{Ndc} 80^{\text {Bonsai }}$ associated with a microtubule. This shortened, $17 \mathrm{~nm}$ long, chimeric construct contained both globular regions of the complex connected by a minimal coiled-coil sequence. In this construct, Ndc80 was fused to Spc25 and Nuf2 to Spc24. Ndc80 ${ }^{\text {Bonsai }}$ allowed for high quality purification of the recombinant variant of the Ndc80 complex necessary for structural studies [93]. Inspection of electron density data from helical reconstruction of cryo-EM samples allowed Alushin et al., 2010 [108] to identify the "toe" region within human Ndc80 protein that recognized a site between two tubulin monomers. Interestingly, this interaction did not discriminate between $\alpha$ - and $\beta$-tubulin, as was previously observed in C. elegans Ndc80 complex [99]. Instead, human $\mathrm{Ndc} 80^{\text {Bonsai }}$ binds to microtubule protofilaments in regular intervals equivalent to a distance between single tubulin monomers (Figure 1.4.B, C). A recent study addressed this discrepancy with near-atomic resolution cryo-EM helical reconstructions of a different human Ndc80 construct, named Ndc80 $0^{\text {Broccoli }}$ (which contained Ndc80/Nuf2 CHD, N-terminal Ndc80 tail and most of the coiled-coil region but lacked Spc24 and Spc25 altogether [110]). This study confirmed the structural differences in binding and specificity towards tubulin monomers between human and C. elegans Ndc80 complexes [109]. Interestingly, the Ndc80 N-terminal tail is not essential in $C$. elegans, while it is crucial in human cells $[49,104-106]$. The unstructured nature of the Ndc80 N-terminal tail did not allow for clear visualization of its interaction with microtubules by cryo-electron microscopy $[99,108,109]$. It was suggested, however, that the $\mathrm{N}$-terminal tail of the human $\mathrm{Ndc} 80$ complex contributed to cooperativity of the interaction and recognition of both straight and curved tubulin 
protofilaments, while the "toe" of CHD contributed to binding exclusively to straight microtubules [108].

In humans, the Ndc80 N-terminal tail is crucial for microtubule attachments. Moreover, post-translational modifications play an important role in regulation of the Ndc80 complex binding to microtubules. The tail region is heavily phosphorylated by Aurora B kinase: of the nine residues in $\mathrm{Ndc} 80$ protein that are phosphorylated in vitro, six sites were confirmed in vivo [93,111-114]. The tail phosphorylation is very prominent in early stages of mitosis, decreasing to zero or one phosphate group per Ndc80 in metaphase $[69,115]$. In vitro studies demonstrated that phosphorylation of the tail decreased the affinity of Ndc80-microtubule interaction $[42,116]$, proportionally to the number of phosphorylated/phosphomimetic residues [69]. This allowed for a graded rheostat-like regulation of $\mathrm{Ndc} 80$ association with microtubules. Similar to in vitro studies, a graded response was observed in cells expressing phosphomimetic mutants of Ndc80 tail. In general, severity of the phenotype was proportional to the number of phosphomimetic residues mutated within the tail. The phenotypes included: defects in chromosome alignment, decrease of inter-kinetochore distances and K-fibers stability, and impairment of chromosome oscillations [69]. 


\section{Figure 1.4 The Ndc80 complex}

(A) Top - Structure of engineered Ndc $80^{\text {Bonsai }} \Delta \mathrm{N}$ construct comprised of globular heads of Ndc80 and Nuf2 (MT binding end) fused by short helical structures with globular domains of Spc24 and Spc25 (Kinetochore binding end). PDB: 2VE7. Bottom - Model of a structure of the full-length Ndc80. Unstructured N-terminal tail of Ndc80 is not shown. Reproduced and modified from [93] with permission (License: 3843340989355)

(B) Electron density map of $\mathrm{Ndc} 80^{\text {Bonsai }}$ bound to the microtubule lattice obtained by high resolution helical reconstructions of cryo-EM micrographs (EMDataBank: EMD-5489) [117]. Green - a single protofilament of tubulin subunits, blue - the Ndc80 subunit, yellow - Nuf2. Purple - unassigned densities that likely correspond to the fragments of the Ndc80 N-terminal tail. The "purple" densities between adjacent Ndc80 molecules proximal to the microtubule lattice (black arrow) may correspond to Zone 1 of the tail (see text below). Second "purple" density is seen on the opposite side of the structure (red arrow) and likely interacts with tubulin E-hook protruding from the adjacent microtubule protofilament. Red - last visible residue of the tubulin subunit, remaining $~ 10-15$ amino acids of the tubulin C-terminal end are predominantly unstructured and not visible on the electron density map (E-hooks).

(C) Crystal structures of two Ndc80 ${ }^{\text {Bonsai }}$ molecules (PDB: 2VE7) and tubulin (PDB: 1JFF) docked into cryo-EM density map, colored as in (B). Reproduced from [117] with permission (License: 3843350991211 ) 
Figure 1.4

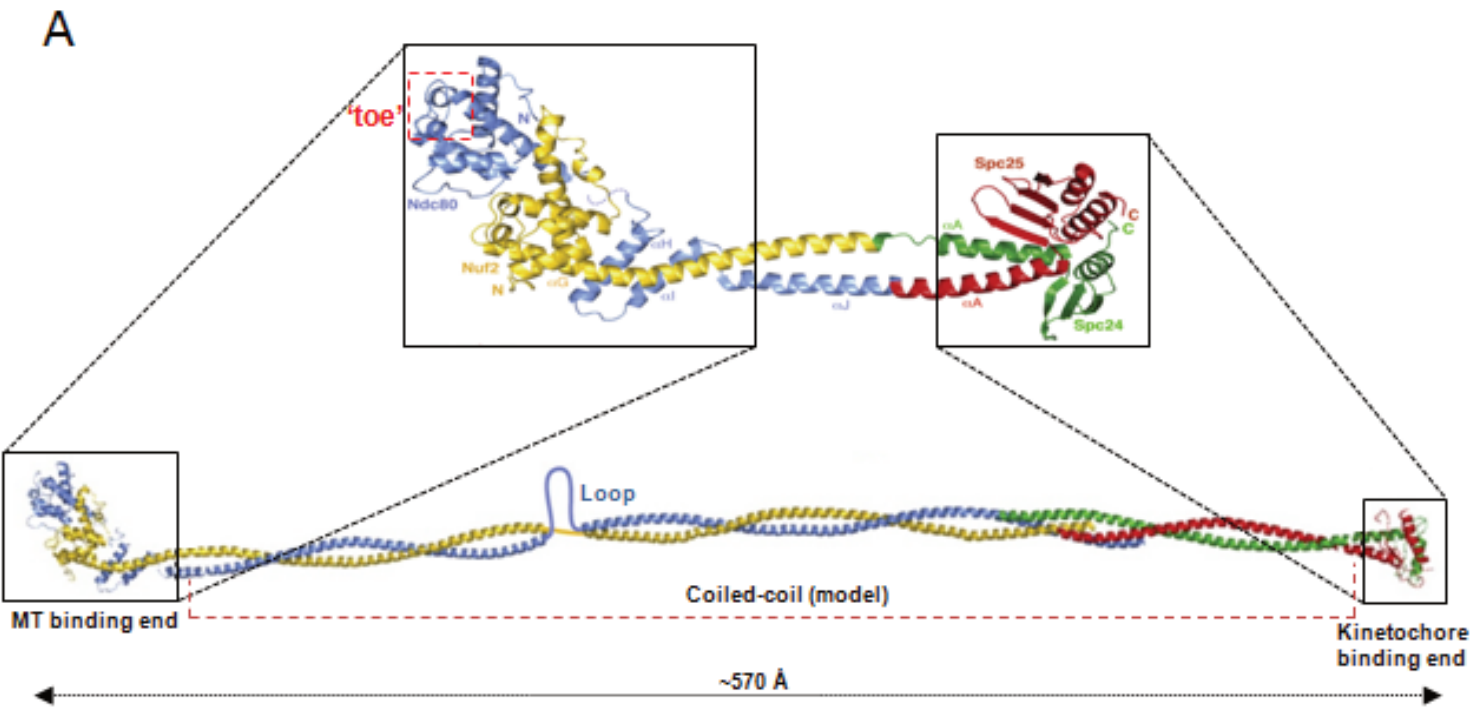

B

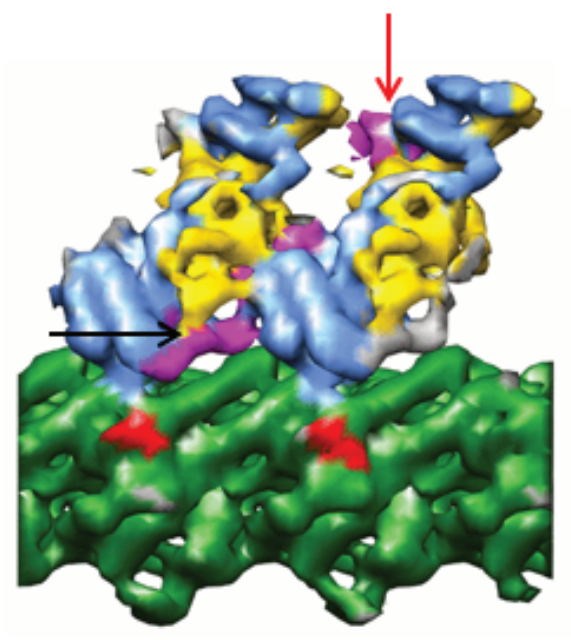

C

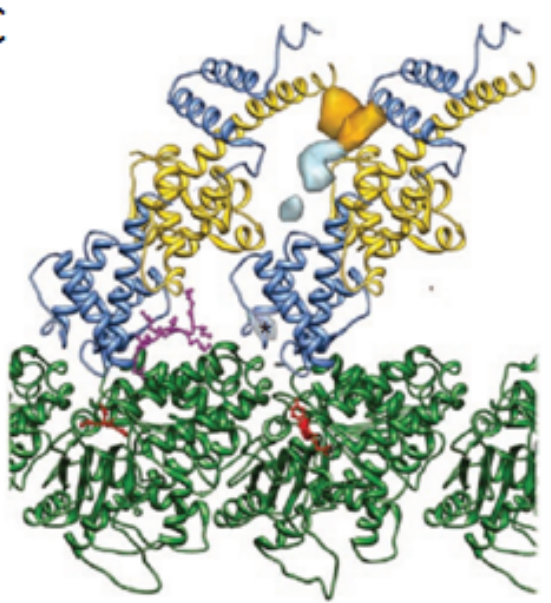


Alushin et al., 2012 [117] proposed a model in which the Ndc80 tail can be divided into two phosphorylation zones that are functionally distinct from each other. Zone 1 is situated between amino acids 44-69, proximal to CHD of Ndc80, while zone 2 is proximal to $\mathrm{N}$-terminus of $\mathrm{Ndc} 80$, encompassing residues $4-15$. The model suggests that the phosphorylation of Zone 1 is responsible for regulation of binding to microtubules, as phosphomimetic mutants within this zone were shown to significantly reduce microtubule binding affinity. Phosphorylation within Zone 2 reduces both the binding affinity and the cooperativity of Ndc80-microtubule interaction, as observed by decreased ability of Ndc80 molecules to form clusters alongside microtubule protofilaments. In agreement with that model, the cryo-EM helical reconstruction of $\mathrm{Ndc} 80^{\text {Bonsai }}$ bound to the microtubules allowed for identification of weak electron densities residing between adjacent $\mathrm{Ndc} 80$ molecules that could not be assigned to $\mathrm{CH}$ domains, but are possibly corresponding to structured regions of the N-terminal tail. Basically, two patches of those densities could be observed. The first patch, which could correspond to Zone 2, is extending to the opposite side of the molecule and has been suggested to interact with the E-hook originating from an adjacent microtubule protofilament. The second patch probably corresponds to Zone 1 and is located between two Ndc80 molecules adjacent to one another on a single protofilament [117] (Figure 1.4B,C). Part of that region can be also observed in the near-atomic resolution structures published recently [109]. It is worth noting that $\mathrm{Ndc80}$ is able to efficiently track depolymerizing ends of microtubules in vitro when it is oligomerized on beads or by antibodies [118], but it does not retain that ability if low, sub-nanomolar concentrations of soluble Ndc80 monomers are used [110,119]. This may suggest that at least some fraction of Ndc80 molecules have to form clusters on microtubules to allow for efficient chromosome congression and segregation. However, a recent study reported only small changes in the cooperativity factor between different phosphomimetic mutants of the 
Ndc80 tail. Additionally, computer modeling based on detailed biophysical characterization of Ndc80-microtubule interactions suggested that only $8 \%$ of $\mathrm{Ndc} 80$ molecules should bind to microtubules as dimers and $2 \%$ as larger clusters [120]. Moreover, sub-nanomolar concentrations of Ndc80 can track microtubule depolymerizing ends when Dam1 complex or Ska complex is present $[119,121]$. This suggests that additional factors may be involved in stabilizing Ndc80-microtubule attachments at the depolymerizing ends, thus allowing for efficient chromosomal movements. To summarize, despite extensive research, it is still unclear whether Ndc80 forms oligomeric arrays on microtubules in vivo, or if those clusters are physiologically relevant for cell functions.

Initial attempts to quantify the number of molecules within the kinetochores using fluorescent tags indicate that the $\mathrm{Ndc} 80$ complex is present in eight copies per microtubule during metaphase in budding yeast [63-65]. Recent, updated measurements place that number closer to twenty Ndc80 molecules per kinetochore microtubule in both budding yeast $(17.4 \pm 2.1)$ and chicken DT40 cells $(18.1 \pm 3.2)[65,95,122]$. In human cells that number is slightly lower, with $14.3 \pm 1.9$ molecules per kinetochore-bound microtubule [95]. The more direct, biochemical estimates using Xenopus laevis egg extracts (meiosis II) suggest $\sim 30 \mathrm{Ndc80}$ molecules per kinetochore microtubule [43]. A number of 15-30 Ndc80 molecules per microtubule attachment, rather than the initial value of $\sim 8$, is mechanistically important for Ndc80 function in creating load-bearing microtubule attachments necessary for chromosome segregation. 


\section{The Ska complex}

One of the most important factors in formation of proper kinetochore-microtubule attachments is the ability to maintain depolymerization-driven movements. The Ndc80 complex has been a leading candidate for a direct coupling factor as it can form loadbearing attachments in vitro when bound on beads or by antibodies [118]. Although Ndc80 can bind to the lattice of straight microtubules, it cannot bind to curved tubulin protofilaments and cannot track depolymerizing microtubule ends when in solution $[110,119]$. This suggests that other couplers might exist in cells that either play a direct role in coupling microtubule depolymerization with chromosomal movements, or facilitate Ndc80 in that role.

One such coupler is known in budding yeast. The 10-protein Dam1 complex (also known as DASH or DDD) is essential for stable attachment formation [123-128]. It directly interacts with microtubules and is able to track depolymerizing microtubule ends both in vivo and in vitro [82,128]. The Dam1 complex forms a ring around the microtubule that allows the complex to "slide" along the depolymerizing plus-end $[128,129]$. It has been shown, however, that the ring formation is not necessary for the complex to exert tracking activity [130]. Interestingly, the Dam1 complex confers this activity to the Ndc80 complex $[119,131]$. The properties described above suggest that in budding yeast the Dam1 complex is the major microtubule coupler. In fission yeast, however, Dam1 is not essential for viability $[64,66,67]$, whereas in vertebrates no homologue of Dam1 was found so far. In vertebrates, one potential candidate has been suggested to function as microtubule coupler and it displays similar properties as the Dam1 complex: the Ska complex.

A proteomic screen of human mitotic spindle identified over 150 potentially novel components of mitotic spindle [132], including C18Orf24, later named Ska1 (ㅁpindle and 
Kinetochore Asssociated protein 1). Further study characterized Ska1 and has shown that it forms a complex with Ska2 (also known as FAM33A) and demonstrated that both are required for mitotic progression [133]. Ska1 and Ska2 have been shown to interact with one another and both localize to kinetochores and co-localized with spindle microtubules. Although kinetochore-associated microtubule fibers (K-fibers) were weakened in Ska1 or Ska2 siRNA-mediated depletion, chromosome congression was not impaired. Knockdown of the Ska complex, however, resulted in prolonged metaphase-like arrest, with rare Mad2-positive kinetochore staining and persistent checkpoint activation. Despite severity of the phenotype, no other proteins appeared to be missing from the kinetochore, suggesting that Ska complex is not essential for kinetochore assembly but may play an important role in coupling of the microtubule attachments to the kinetochore. This discovery prompted increased interest aimed to further characterize this potentially important complex. Only in 2009, multiple crucial studies were published [121,134-137], demonstrating significant functions of that complex. Five of those studies independently identified a third member of Ska complex, Ska3 (initially named Rama1 [121,136] or C13orf3 [134,135,137]). Below I discuss the structure, known functions and the importance of the Ska complex for cell division.

The kinetochore localization of the Ska complex can be observed in prometaphase, however, it is most prominent during metaphase [121,133,134,136-139]. Its recruitment to the kinetochore is dependent on the $\operatorname{KMN}$ network $[121,133,136,139]$, and it has been suggested that the Ndc80 "kink" region plays a role in this process [103]. Different groups reported somewhat inconsistent phenotypes of siRNA mediated depletions of the Ska complex components: either transient chromosome alignment defects followed by mitotic delay or arrest $[134,137]$ or persistent chromosome alignment defect $[121,135,136]$. Recent study conducted using live cell imaging demonstrated that the majority of the cells properly aligned chromosomes on the metaphase plate, although 
a few chromosomes exhibited alignment delays in some cells [138]. Moreover, the rare "escaper" phenotype could be observed in a small number of cells, as first described by Hanisch et al., 2006 [133]. The most prominent effect of Ska depletion was the prolonged metaphase arrest that eventually led to cohesion fatigue and asynchronous separation of sister chromatids $[138,140]$. It is worth noting that the Ska depletion phenotypes were best observed in cells treated with pooled, multiple siRNAs targeting the combination of Ska complex members.

Aside from the role in formation of stable kinetochore-microtubule attachments and maintenance of sister chromatid cohesion, the Ska complex has been implicated in silencing the spindle checkpoint $[133,137,138,141]$. Proteomic studies in Ska3 ${ }^{-/-}$knockout chicken DT40 cells indicated a reduction in levels of chromosome-associated APC/C

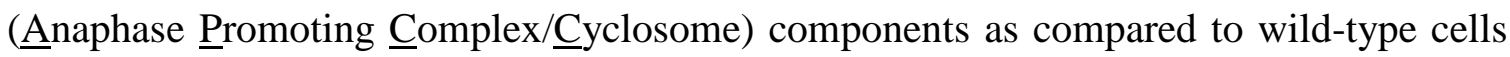
[141]. Moreover, depletion of the Ska complex in human HeLa cells results in similar decrease of APC/C on chromosomes, while forced localization of the Ska complex onto kinetochores promotes APC/C localization to chromosomes, leading to enhanced anaphase onset [138]. Mitotic delay was still observed in Ska depleted cells treated with Mps 1 inhibitor reversine. This suggests that Ska function in checkpoint signaling may be downstream of microtubule attachment formation, which displaces Mps1 from kinetochores and promotes mitotic progression [142,143].

Biochemical analysis of the human Ska complex has shown that Ska1 and Ska2 form a heterodimeric subcomplex that further dimerize in the presence of Ska3 [121], resulting in heterohexameric assembly. Ska3 has been shown to form a binary complex with Ska1 in the absence of Ska2, but not with Ska2 alone [135]. N-terminal regions of all three components are predicted to form coiled-coil structures and the high resolution X-ray crystallographic studies of the recombinant human Ska1 $\Delta \mathrm{C}-\mathrm{Ska} 2-\mathrm{Ska} 3 \Delta \mathrm{C}$ 
(a minimal oligomerization unit) demonstrated a W-shaped structure with two triple helical bundles (Figure 1.5.A, B). This construct forms a central core of the complex that is characterized by the presence of negatively charged patches on the solvent accessible surface. Mutations introduced within the dimerization interface (F7R/L11R in Ska3) increase the Stokes radius but do not block the dimerization of the complex. Interestingly, different structural arrangement of the Ska1-Ska2-Ska3 ${ }^{\text {F7R/L11R }}$ dimer does not influence the localization of the complex to the kinetochore but causes a loss of function as this mutant cannot rescue the mitotic delay caused by the depletion of the wild-type complex [144]. It may indicate that the topography of the complexes core is important, even though the major microtubule binding region resides outside the core.

The Ska complex contains a microtubule binding domain (MTBD) within Cterminal region of Ska1. Mutations that disrupt the positively charged patches on its surface significantly reduce its microtubule binding affinity. MTBD allows the complex to interact with both straight microtubules and curved tubulin protofilaments, albeit with rather weak affinities $(\sim 2.5 \mu \mathrm{M})[18,110,145]$. This distinguishes Ska from the Ndc80 complex, as the latter preferentially binds to straight microtubules, showing only a very weak affinity towards curved protofilaments [110]. Also the mechanism of microtubule interaction is significantly different between those complexes: Ndc80 has defined microtubule binding regions ("toe" and N-terminal tail) and orientation in which it assembles on the microtubule lattice $[99,108,109]$, whereas Ska1 MTBD contains multiple binding surfaces that are able to bind to the microtubule lattice in various orientations (Figure 1.5.C) [145]. The Ska complex induces microtubule bundling at high concentration, as shown by light and electron microscopy [121,144] (and unpublished data). It is not uncommon for structures containing two microtubule binding domains to induce bundling by bridging microtubules, however, the Ska1-Ska2 sub-complex displays the bundling activity at a similar range of concentrations as a full Ska complex 
[121]. This suggests a different mechanism for bundling microtubules, as Ska1-Ska2 subcomplex contains only one MTBD.

The ability to bind curved protofilaments [110,145] allows the Ska complex, either on beads or in solution, to track the depolymerizing microtubule ends. The similarities between the Ska complex and Dam1 complex are also visible in their ability to accumulate $\mathrm{Ndc80}$ at the plus end of depolymerizing microtubules [110]. It is currently believed that the Ska complex behaves like a functional homologue of the Dam1 complex in vertebrates. Interestingly, presence of the Ska complex increases the affinity of the Ndc80 complex towards microtubules. Similarly, Ska binding to microtubules is increased by the Ndc80 complex, indicating that the complexes bind to the microtubules synergistically and possibly stabilize each others interactions with the depolymerizing ends [110]. It also suggests a possible direct interaction between Ska and Ndc80, however, it has not yet been demonstrated.

Despite significant progress in structural and functional characterization of the Ska complex, there is little known about $\sim 300$ amino acid long C-terminal domain of Ska3. It contains low-complexity sequences and so far has been implicated in contributing to microtubule binding. It has been also suggested to play a role in the recruitment of the Ska complex to the kinetochores [144]. However, no detailed studies were carried out to further characterize this region. 


\section{Figure 1.5 The structure of the Ska complex}

(A) Structure of the Ska core complex (Ska1 $\Delta \mathrm{C}$-Ska2-Ska3 $\Delta \mathrm{C})$ [144]. N-terminal helical bundle intercalates with its dimeric counterpart to form a W-shaped dimer. PDB: 4AJ5 Chains A, F, L, P, V, Z. Visualization by PyMOL (Schrödinger).

(B) Surface representation of the Ska core complex electrostatic potential. Multiple negatively charged patches can be observed, in agreement with theoretical pI of 5.1. Indicated residues of Ska1 (green), Ska2 (blue) and Ska3 (orange) contribute to the highlighted charged patches (ovals). Reproduced and modified from [144] with permission (License: 3835711472313 )

(C) Cartoon representation of the human Ska1-MTBD where surface-exposed K/R residues are shown as sticks (left). Surface representation of the Ska1-MTBD in the same orientation with electrostatic surface potential revealing the presence of positively charged patches (right). Green, yellow and blue labels indicate patches of residues shown to contribute to the interaction with microtubules. PDB: 4C9Y. Reproduced and modified from [145] under Creative Commons Attribution 3.0 Unported License. 
Figure 1.5

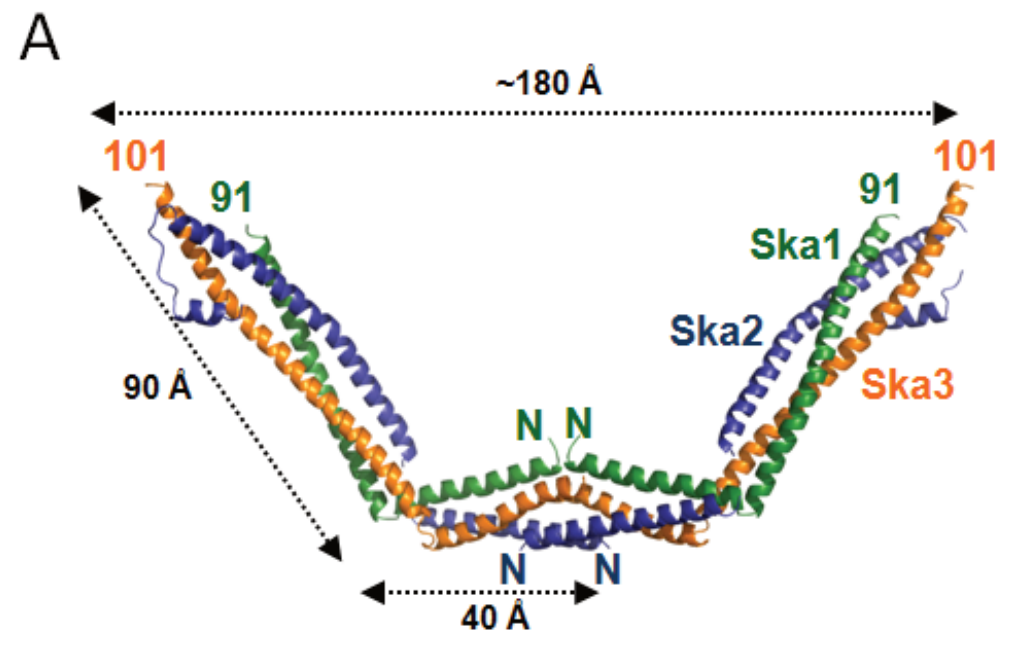

B
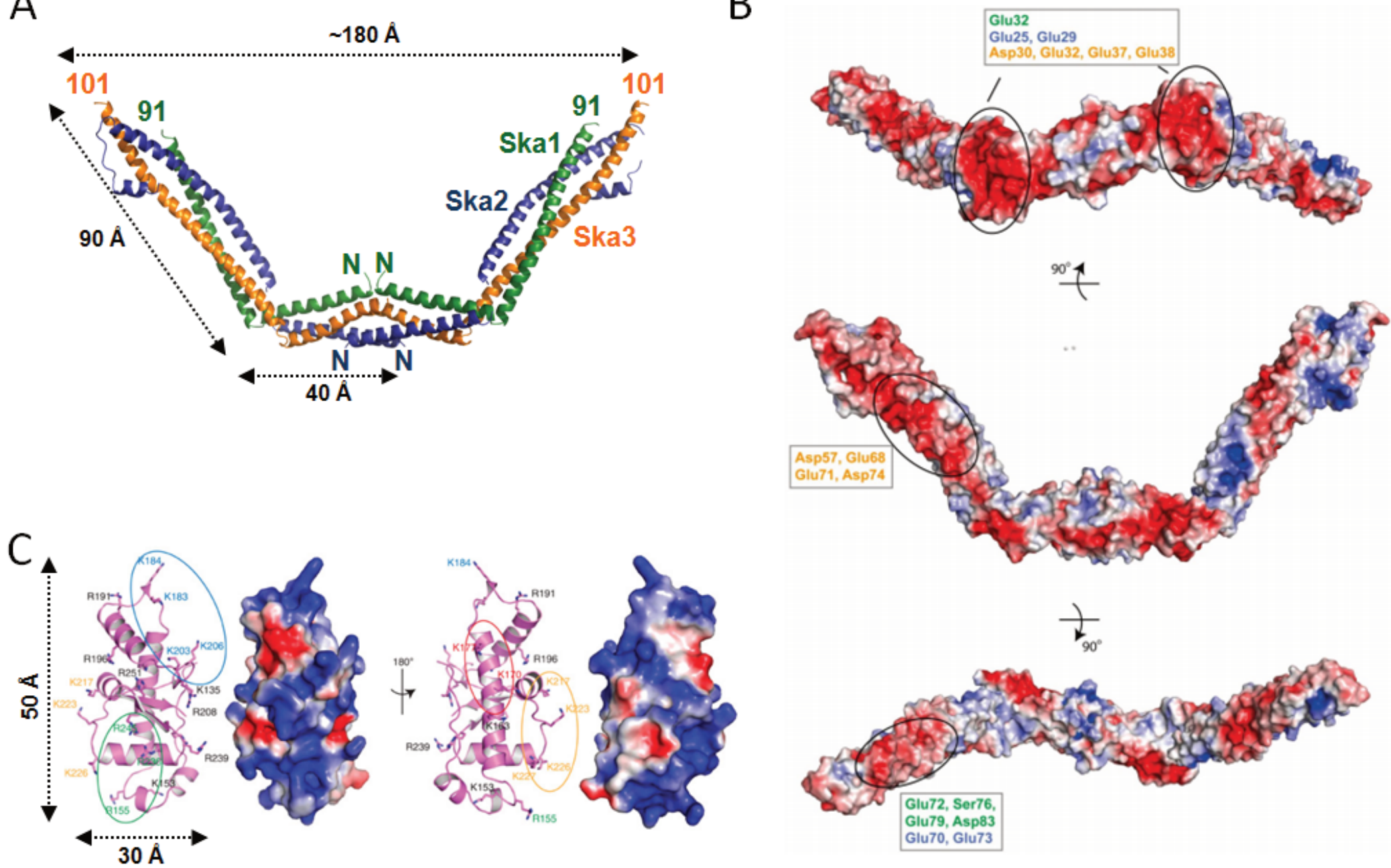


\section{Maturation of kinetochore-microtubule attachments}

Maturation of the kinetochore-microtubule attachments can be defined as a process resulting in formation of stable end-on attachments capable of carrying chromosomal load. But what exactly makes the attachments "stable" and how to determine the stability of the attachment? The most popular technique used to assess the stability of the microtubule attachments is the "cold-stability" assay based on the phenomenon of cold-driven microtubule depolymerization. In mitotic cells, microtubules that have formed end-on attachments with kinetochores are protected from massive depolymerization and thus withstand the cold treatment [146,147]. Nevertheless, it is important to note that this assay does not give a simple, binary answer. For example, siRNA-mediated depletion of Ska3 results in $~ 20 \%$ drop in intensity of fluorescently stained kinetochore-associated microtubules, while $\sim 60 \%$ reduction can be observed when the Ndc80 protein is depleted [135]. This graded stability of the attachments likely suggests that the maturation of the end-on attachments is a process that requires multiple steps facilitated by various components.

Although the mechanism of kinetochore-microtubule attachment maturation is still unclear, multiple essential components have been extensively studied and characterized. The most prominent complex required for formation of end-on attachments is the Ndc80 complex, described above. Importantly, the stability of the attachments is regulated by Aurora B-dependent phosphorylation of the Ndc80 tail, creating a rheostatlike graded response $[42,69,116]$. One of the current models of that regulation derives from the observation that the Aurora B activity diminishes with increasing distance from the inner-centromere [148]. An attachment that is capable of increasing the interkinetochore stretch would therefore further strengthen itself by reducing the Aurora B-driven phosphorylation of the Ndc80 tail. Although Ndc80 is essential to form end-on 
attachments, other proteins were shown to contribute directly or indirectly, e.g. the Ska complex $[110,121]$. However, how Ska interacts with Ndc80 on the microtubule and facilitates depolymerization driven movements remains elusive. Interestingly, cells depleted of Ska can still align chromosomes properly, but fail to satisfy the spindle checkpoint $[137,138]$. This may indicate that Ska is dispensable for the formation of initial end-on attachments, but is crucial in subsequent maturation steps required for attachment stabilization and SAC silencing.

\section{Spindle Checkpoint}

Mitotic division requires a very stringent safeguard mechanism to prevent premature chromosome segregation. Cells that transit to anaphase before all chromosomes are bi-oriented and aligned on the metaphase plate, separate their genetic content unevenly between daughter cells leading to abnormalities in the chromosomal content, often observed in cancer cells. The mechanism that ensures faithful chromosome segregation, called Spindle Assembly Checkpoint (SAC or Spindle Checkpoint), blocks the transition from metaphase to anaphase until all chromosomes are properly positioned and have formed stable microtubule attachments. SAC creates a binary response, in which Cdc20, a cofactor of APC/C is inactivated until all SAC requirements are satisfied [149-152]. In absence of spindle checkpoint signal Cdc20 activates APC/C to target securin and cyclin B for ubiquitin-dependent proteolysis [153-156]. Degradation of securin reactivates separase that in turn cleaves the cohesin complex, effectively disrupting the connection between sister chromatids and allowing for their separation. Proteolysis of cyclin B leads to inactivation of the CDK1 kinase and results in dephosphorylation of CDK1 substrates, inducing mitotic exit. 


\section{Mechanism of Spindle Checkpoint signaling}

Spindle checkpoint is initiated on unattached kinetochores by production of a diffusible mitotic checkpoint complex (MCC), which is composed of Bub3, Mad2, BubR1 (Mad3 in yeast, worms and plants) and Cdc20 [157-159]. When Cdc20 becomes a part of MCC it can bind to, but is unable to activate the APC/C.

The kinetochore acts as a regulator and a platform for assembly of the SAC machinery. The Mps1 kinase directly binds to the CHD of Ndc80 on the unattached kinetochores in Aurora B-dependent manner [142,143,160-162]. Phosphorylation of multiple MELT (methionine-glutamate-leucine-threonine) motifs of Knl1 by the Mps1 kinase in prometaphase facilitates recruitment of Bub1, BubR1 and Bub3 [162-170]. Bub1 is phosphorylated by Mps1, promoting kinetochore localization of the Mad1-Mad2 complex [171] and contributing to Aurora B recruitment to the inner centromere [172]. In metazoans, Bub1 is also required for recruitment of the RZZ complex (Rod-ZW10Zwilch) that contributes to kinetochore recruitment of Mad1-Mad2 [173-177]. Mad2 exists in two distinct topologies: the "open" conformer that, upon binding with either Mad1 or Cdc20, undergoes a conformational change to the "closed" state that sequesters Cdc20 and can form the MCC complex, effectively inhibiting APC/C [178-182]. Kinetochore-associated Mad2 catalyzes the conformation transition from open to closed state of unbound Mad2 molecules, which can subsequently bind to Cdc20, allowing for strong amplification of the spindle checkpoint signal $[183,184]$. It is an efficient way to prevent $\mathrm{APC} / \mathrm{C}$ activation and it creates a very responsive mechanism capable of preventing mitotic exit even when only single kinetochore remains unattached. 


\section{Silencing of spindle checkpoint signal}

When all chromosomes are bi-oriented and aligned on the metaphase plate, spindle checkpoint signal is turned off and the cell can progress to anaphase. For efficient silencing of checkpoint signal kinetochore-associated SAC proteins have to be inactivated or removed. Predominant mechanism for the removal of spindle checkpoint proteins is known as "stripping" by the dynein motor. Cytoplasmic dynein is a minus-end directed motor that binds to kinetochore-bound microtubules and actively carries the SAC proteins away from the kinetochore [46]. Recruitment of dynein to the kinetochores is facilitated by Spindly, a protein associated with the RZZ complex [177,185]. Although depletion of Spindly prevents dynein recruitment to kinetochores, Mad1 and Mad2 are still removed, albeit at much lower rate. This suggests the existence of another, dyneinindependent mechanism for SAC removal that could be evolutionary conserved, as fungi and plants lack kinetochore-associated dynein [186]. This mechanism is likely to involve protein phosphatases that oppose Mps1 and prevent recruitment of new SAC proteins $[187,188]$.

When unattached, kinetochores signal for mitotic arrest and MCC is constantly produced by SAC. To allow for anaphase transition, inhibition of APC/C has to be removed. This can be achieved by several mechanisms. One of them involves APC15, an APC/C subunit, that targets MCC for degradation by Cdc20 ubiquitylation [189-191]. Disassembled MCC is quickly replaced by another one while spindle checkpoint remains active as Cdc20 is constantly synthesised and new "closed" Mad2 is formed. This creates a system with high turnover rates, allowing for rapid transition to anaphase once SAC proteins are removed from the kinetochore and no new MCC is formed.

Formation of properly oriented end-on kinetochore-microtubule attachments is necessary to silence the spindle checkpoint. It is still unclear, however, what defects are 
sensed by the SAC. A classic study demonstrated that application of force to a monovalent polar chromosome was able to satisfy the checkpoint [192] giving rise to the notion that SAC recognizes the tension between sister chromatids. Subsequent experiments have shown that applied tension allows for stabilization of the microtubules attachments. It suggested that formation of mature kinetochore-microtubule attachments is necessary to silence the checkpoint [193]. Subsequent studies provided enormous insight into so called "tension checkpoint" and "attachment/occupancy checkpoint", however, it remains unclear if those mechanisms work together or only one of them is essential. Two recent studies utilized a Ndc80 mutant that cannot be phospho-regulated by Aurora B (Ndc80-9A) and thus allows for efficient binding with microtubules. Human cells expressing this mutant were able to form stable microtubule attachments in monopolar spindles and exit mitosis despite the lack of both bi-oriented chromosomes and interkinetochore stretch [194,195], showing that the microtubule attachment is sufficient for cells to proceed to anaphase. Another study demonstrated that cells treated with taxol (microtubule stabilizing drug that reduces the stretch) can transition from metaphase to anaphase only if all kinetochores formed microtubule attachments [68], supporting the notion that tension is not essential for SAC silencing. It was suggested, however, that tension-induced changes in kinetochore architecture may influence the efficiency or speed of silencing. For example, the increase in a distance between Ndc80 and Knl1 molecules upon microtubule attachment restricts Mps1 (bound to Ndc80) from its substrate (Knl1) and serves as an essential mechanical switch for SAC silencing in budding yeast [196]. It is possible that in human cells that mechanism could aid in countering low levels of Mps1 remaining on attached kinetochores [142,197]. The stretch was also suggested to physically separate inner-centromere localized Aurora B from its multiple substrates in the outer kinetochore, as Aurora B kinase activity decreases 
proportionally to the distance from the inner-centromere [148]. Microtubule binding complexes $\mathrm{Ndc} 80$ and Ska, both required for formation of proper microtubule attachments, are down-regulated by Aurora B phoshorylation. Additionally, the binding of protein phosphatase 1 (PP1) to Knl1 is prevented by Aurora B phosphorylation $[187,198]$. Role of PP1 in SAC silencing is described later.

\section{Recognition of the mature kinetochore-microtubule attachments by}

\section{the Spindle Checkpoint}

How are mature end-on kinetochore-microtubule attachments recognized by the spindle checkpoint machinery? Although the mechanism remains elusive, the prevailing model places the Mps1 kinase as the potential sensor for detecting microtubule attachments. First of all, the Mps1 kinase is one of the key upstream regulators of SAC signaling as Mps1-dependent phosphorylation of MELT motifs on Knl1 facilitate recruitment of SAC components to the kinetochore. Inhibition of Mps1 results in mitotic exit in arrested cells suggesting a major role in SAC signaling [199-201]. Additionally, Mps 1 was also implicated in facilitating Aurora B recruitment to the inner centromere through Bub1 and Shugoshin (Sgo) positive feedback loop [172]. Moreover, Mps1 was shown to enhance Aurora B activity by phosphorylating its regulatory subunit Borealin/Dasra B [161]. Mps1 directly associates with Ndc80 and was shown to be displaced from the kinetochore upon microtubule attachment [142,143]. Those characteristics of Mps1 place it as not only a major component in initiating SAC signaling, but also as a possible sensor of the kinetochore-microtubule attachment status. However, for efficient SAC silencing, the removal of the kinase has to be followed by the reversal of the phosphorylation events. This is accomplished by protein phosphatases that were shown to oppose Mps1 and allow for mitotic exit $[187,188]$. 
Two major protein phosphatases found on kinetochore have been implicated in SAC silencing: PP1 and PP2A. In mitosis PP1 is recruited to the kinetochores by SILK and RVSF motifs of Knl1 and this interaction is inhibited by Aurora B phosphorylation on the RV $\underline{\mathbf{S} F}$ motif $[187,198]$. Knl1 mutants that cannot bind PP1 are inefficient in spindle checkpoint silencing [187,188,198,202]. Multiple studies suggested that PP1 activity counters Aurora B functions [203-207]. The second phosphatase, PP2A-B56, is recruited to outer kinetochores by interaction with BubR1 that is indirectly associated with Knl1 [208-211]. It has been suggested that PP2A-B56 dephosphorylates RVSF motif of Kn11, thus allowing for PP1 recruitment [188]. Both PP1 and PP2A have been implicated in reversal of the Mps1-dependent phosphorylation of MELT motifs of Knl1 [188,212]. Interestingly, this creates a negative feedback loop: PP2A promotes PP1 recruitment, which in turn reduces Mps1 phosphorylation of MELT motifs, effectively inhibiting further recruitment of PP2A. It is likely that this feedback loop allows for responsiveness of SAC: on unattached kinetochore PP2A is primed to efficiently facilitate PP1 recruitment/MELT dephosphorylation as soon as kinase activity is reduced (e.g. by stretching and/or Mps1 displacement by microtubules), which in turn removes PP2A to prepare the system for quick response if mitotic kinases are reactivated.

The studies described above led to a model in which Mps1, together with PP1/PP2A associated with Knl1, could serve as a switch capable of triggering SAC silencing by sensing microtubule attachment status. Once microtubule is attached the displacement of Mps1 should shift the equilibrium between kinases and phosphatases towards the latter. PP1 localization to Knl1 would increase, initiating dephosphorylation of mitotic substrates and consequent SAC silencing. However, it is still unclear how the Mps1 would trigger the shift towards dephosphorylation, as inhibition of Mps1 does not result in the reduction of the Aurora B kinase activity [199-201]. The model also fails to 
explain a modest, only 10 min long, delay in mitosis caused by the replacement of the wild-type Knl1 with a truncation mutant unable to bind PP1 [166]. It is possible that other kinetochore components contribute to association of the phosphatases at the kinetochores. A likely scenario could also involve an additional sensing mechanism to recognize mature attachments. One of the possible candidates is the Ska complex that was recently suggested to act downstream of Mps1 in SAC silencing [138]. Furthermore, Ska depletion causes a robust mitotic arrest that requires active SAC. In summary, the ability of the Ska complex to bind curved microtubule protofilaments, contributing to Ndc80microtubule binding and tracking depolymerizing ends suggest that Ska could be an important factor in recognizing mature attachments and triggering SAC silencing. 


\section{Chapter II}

\section{The unstructured tail of Ndc80 recruits Ska to kinetochores to facilitate exit from mitosis}

This chapter is based on the following manuscript (in preparation).

Janczyk, P.Ł., Skorupka, K., Tooley J., Matson, D.R., Miller, S., Kestner, C., West, T., Pornillos, O. and Stukenberg, P.T. "The unstructured tail of Ndc80 recruits Ska to kinetochores to facilitate exit from mitosis" 


\section{$\underline{\text { Abstract }}$}

Kinetochores bind microtubules of the spindle and couple this attachment to the silencing of the spindle checkpoint signal that blocks the metaphase to anaphase transition. This is in part accomplished by the same domain of the Ndc80 protein directly binding microtubules and recruiting the Mps1 kinase that generates the signal. To silence the Spindle Assembly Checkpoint (SAC) the Ska protein must also recruit the PP1 phosphatase to kinetochores. It is not understood how Ska is recruited to kinetochores and how this is linked to microtubule attachments. We characterize a novel mutant of the Ndc80 unstructured tail that allows proper kinetochore microtubule attachments and the generation of full pulling forces, but prevents mitotic exit. Ska is not recruited to the kinetochore in cells expressing this mutant. The mutant can bind microtubules with similar affinity as the wild type protein in vitro, but poorly clusters along protofilaments of bound microtubules. In addition the mutant displays apparent negative cooperativity in equilibrium binding assays consistent with its inability to cluster on microtubules. Ska increases the rate of dephosphorylation of the $\mathrm{Ndc} 80$ tail by PP1 in vitro. By electron tomography we observe characteristic V-shaped structures that resemble the Ska complex in both shape and size, positioned along microtubule protofilament in presence of both wild-type Ndc80 and Ska. Directly underneath those structures we identified Ndc80 clusters, suggesting that the clustering of Ndc80 subunits is required to recruit Ska. Our data identify a new function of the Ndc80 tail to regulate the recruitment of Ska protein to control the exit from mitosis. We suggest that these mechanisms act downstream of Mps1 displacement to allow kinetochores to mature end-on attachments and couple the binding of microtubules to spindle checkpoint silencing. 


\section{$\underline{\text { Introduction }}$}

The kinetochore regulates at least three crucial processes in chromosome segregation. It directly attaches to both chromatin and microtubules to link chromosomes to the spindle; regulates microtubule dynamics to generate the force that moves chromosomes; and unattached kinetochores generate the spindle assembly checkpoint (SAC), which blocks the progression to anaphase [11,213-216]. How kinetochores link microtubule binding to spindle checkpoint silencing is a critical unanswered question. While kinetochores contain over 100 proteins, a key finding was that the Ndc80 protein was required to both bind microtubules and generate the SAC signal suggesting that it links the two processes together $[85,217,218]$. Recently it was shown that the Calponin homology domain (CHD) of Ndc80 (also called hsNdc80 or Hec1 in humans) subunit either binds the Mps1 kinase that generates the SAC signal or the lateral side of a microtubule suggesting that microtubule binding, in part, silences the SAC by displacing the Mps1 kinase from the kinetochore. However, the subsequent steps downstream of Mps1 displacement to silence the signal are still poorly understood.

The Ndc80 complex is a heterotetramer composed of the following proteins: Ndc80, Nuf2, Spc24 and Spc25 [83,86,87,219]. N-terminal Calponin homology domains of Ndc80 and Nuf2 directly bind the lateral sides of microtubules and these attachments are critical for chromosome movements. These CHD are connected to globular domains of Spc24 and Spc25, which bind central kinetochore proteins, through a $~ 50 \mathrm{~nm}$ coiledcoil. The binding of the CHD to microtubules is regulated by an unstructured and positively charged 80 amino acid N-terminal tail of Ndc80. Aurora B phosphorylates the Ndc80 tail on 8-12 sites and the amount of phosphorylation correlates with weaker interaction to allow dynamic regulation of microtubule-kinetochore attachments in a rheostat like manner $[69,108,117]$. In prometaphase the Ndc80 tail is heavily 
phosphorylated, while in metaphase it has zero or one phosphorylated residues, which is believed to allow microtubule attachments to mature as mitosis progresses [69]. Kinetochores that are aligned at the metaphase plate are physically pulled toward the poles separating the Ndc80 tail from the Aurora B kinase. However, how phosphatases are recruited to the tail to dephosphorylate the tail is not understood.

Each kinetochore has at least 7 Ndc80 complexes per attached microtubule [6365,95]. Ndc80 complexes can bind along protofilaments in clusters in vitro, but current models suggest that the Ndc80 complex binds as single entities in vivo. Clustering in vitro is mediated by C-terminal region of the Ndc80 tail, which sits between CHDs of adjacent subunits [117]. This region also directly binds microtubules, is phosphorylated on multiple sites by Aurora B and dephosphorylation of this zone is required for proper alignment of chromosomes to the metaphase plate. Phosphorylation of the tail has minimal effect on the clustering of $\mathrm{Ndc} 80$ molecules along microtubules in vitro. The $\mathrm{N}$ terminal half of the tail is similarly phosphorylated by Aurora B and phosphomimetic mutants were also unable to properly align chromosomes in vivo. Because the phosphomimetics block microtubule binding of $\mathrm{Ndc80}$ in vitro and chromosome congression in vivo, but have little effect on the cooperativity of binding [120] it is difficult to use these mutants to test the importance of clustering to Ndc80 function in vivo.

The Ska complex (Ska) is enriched on kinetochores of aligned chromosomes and is involved in generating stable kinetochore-microtubule attachments. Its central function is to recruit PP1 to kinetochores [220]. Ska forms a W-shaped structure [144], composed of Ska1, Ska2 and Ska3 proteins [121,133-137,141]. Ska directly binds microtubules and has been shown to track microtubule depolymerizing plus-end [110,121]. Depletion of any of any protein of the Ska complex results in prolonged, SAC dependent, metaphase 
arrest and eventually cell death [121,133-138]. Despite lack of sequence or structure similarity, it has been suggested that the Ska complex may be a functional equivalent of the yeast Dam/DASH complex $[18,135,145]$. The recruitment of Ska to the kinetochore is dependent on a KMN (Knl1-Mis12-Ndc80) network [121,133,136,139], and is regulated by Aurora B kinase [139]. Ndc80 and Ska have not been shown to interact in vitro, but Ndc80 can decrease the binding affinity of Ska to microtubules [110], suggesting that they may form a complex on microtubules. However, the proteins that recruit Ska to kinetochores or the events that allow recruitment are not known. Previously, an internal loop (also called "kink") within the coiled-coil region of the Ndc80 complex has been shown to associate with yeast Dam/DASH complex [103]. Another study, however, did not observe a similar phenotype with Ska when human Ndc80 loop mutant was used [88].

Here, we generated a number of mutants in the unstructured tail of $\mathrm{Ndc} 80$ to identify novel functions of this regulatory region. Cells expressing two different mutants in amino acids 40-60 of Ndc80 allow congression to the metaphase plate, generate full kinetochore pulling forces, but are unable to enter anaphase. We focused on a construct with a set of point mutants that change the charged residues in this region, which is referred to as $\mathrm{Ndc} 80^{+4 \mathrm{CT}}$. Cells expressing $\mathrm{Ndc}^{+4 \mathrm{CT}}$ are unable to recruit the Ska complex to kinetochores, suggesting a novel function of the Ndc80 N-terminal tail. Cells expressing $\mathrm{Ndc} 80^{+4 \mathrm{CT}}$ neither recruit Ska to kinetochores of aligned chromosomes, nor to kinetochores in cells treated with nocodazole and Aurora B inhibitors suggesting that this region is required for all of the known kinetochore-recruitment pathways of Ska. The $\mathrm{Ndc} 80^{+4 \mathrm{CT}}$ complex has a similar $\mathrm{K}_{\mathrm{d}}$ for microtubule binding in vitro but looses the cooperative binding by both equilibrium binding assays and direct visualization of $\mathrm{Ndc} 80$ clusters on microtubules by EM. Moreover, we identified V-shaped structures along microtubule protofilaments in presence of wild-type Ndc80 and Ska. Moreover, we 
observed Ndc80 clusters positioned underneath those structures that resemble the Ska complex by both shape and size. Altogether, our data suggest that Ska binding is linked to the attachment of kinetochores to microtubules through the Ndc80 tail. In addition it suggests that the clustering of $\mathrm{Ndc} 80$ along protofilaments is not required to align chromosomes to the metaphase plate or to generate kinetochore tension. However, clustering appears to be the key event to recruit Ska/PP1 to trigger the exit from mitosis. 


\section{$\underline{\text { Results }}$}

\section{Positive charge in the $\mathrm{N}$-terminal region of the $\mathrm{Ndc80}$ tail is critical for chromosome}

congression in vivo

The role of phosphorylation of the Ndc80 tail has been extensively studied and it has been established that it regulates the dissociation of $\mathrm{Ndc} 80$ from microtubules in a rheostat like manner $[69,108,115-117,120]$, but has little effect on the clustering of Ndc80 molecules along protofilaments [120]. We were interested if the tail has roles other than regulating microtubule attachments, so we focused on mutations of residues that are unrelated to phosphorylations. We began by generating a deletion set of the Ndc80 tail shortening the tail by 10 amino acids from the $\mathrm{N}$-terminus. We assayed for kinetochore function in vivo using a protocol that allows us to visualize the first mitosis expressing the rescued Ndc80 mutant after depletion of the endogenous protein by siRNA and rescue by transfection (Supplemental Figure 2.1.A).

There was a length dependent decrease in the ability of cells to align chromosomes to the metaphase plate. However, cells with only 20 amino acid long tails were able to align chromosomes to the metaphase plate (albeit with lower efficiency), generate cold stable kinetochore microtubule attachments, and generate interkinetochore tension. Cells expressing only 10 amino acid tails or no tails lost all of these kinetochore functions (Supplemental Figure 2.1.B-E), although the kinetochores remained intact (Supplemental Figure 2.2). This suggests that the essential functions in the tail to generate kinetochore microtubule attachment lie in the amino acids between 61-80. This region has only one aurora B phosphorylation site, does not bind microtubules (data not shown) and is significantly shortened, arguing that these are not the essential aspects of the tail to allow the Ndc80 complex to generate end-on attachments. 
To identify the unappreciated functions in the Ndc80 tail we first asked if the region spanning residues $61-80$ is essential. We used two Ndc80 deletion mutants: $\mathrm{Ndc} 80^{\Delta 60-80}$ and $\mathrm{Ndc} 80^{\Delta 40-80}$. These mutants aligned chromosomes and generated kinetochore-microtubule attachments with similar efficiency as cells rescued with the full Ndc80 protein (Figure 2.1.B-D). Thus the region spanning residues 61-80 is not essential for microtubule attachments.

Under physiological conditions, the Ndc80 tail has 5 negatively and 15 positively charged residues. These are fairly equally distributed along the length of the tail. We asked if the charge is important for kinetochore function. We mutated 10 of the basic amino acids in the tail to alanines to generate a tail with net charge of zero. This neutral tail was unable to align chromosomes to the metaphase plate, generate cold-stable kinetochore attachments or generate inter-kinetochore pulling forces on chromosomes arguing that the charge of the tail is essential for microtubule binding (Supplemental Figure 2.1.A-E). This conclusion is consistent with the fact that Aurora phosphorylation would neutralize this charge to reduce microtubule binding. It is important to note that the elimination of the basic residues also affects Aurora B phosphorylation sites, which require a basic residue in -2 position relative to the phosphorylated serine or threonine, however the elimination of the phosphorylatable residues increases rather than decreases the kinetochore microtubule binding in vivo.

\section{The charge in the $\mathrm{C}$-terminal region of the tail is required to silence the spindle checkpoint.}

We next generated a series of internal deletion mutants of the Ndc80 tail (Figure 1A). We defined metaphase as having the majority of chromosomes aligned in the center of the cell; mitotic cells with more than five unaligned chromosomes were scored as late prometaphase. Our system could rescue Ndc80 depletion with reasonable fidelity as more 
than $16 \%$ of $\mathrm{Ndc} 80^{\mathrm{WT}}$ cells had also achieved proper chromosome alignment, as compared to $19 \%$ of the mock-transfected mitotic cells (Figure 2.1.B and C). Either half of the tail was sufficient to allow microtubule binding. Similarly, all four Ndc80 tail truncation mutants tested were able to align a significant portion of their chromosomes at the time of the fixation, but not as efficiently as the wild type controls. While both the $\operatorname{Ndc} 80^{\triangle 40-80}(17.0 \pm 3.5 \%$ of mitotic cells scored as aligned $)$ and the $\operatorname{Ndc} 80^{\Delta 40-60}(17.7 \pm$ $2.1 \%$ ) mutants aligned chromosomes at a similar frequency to the $\mathrm{Ndc} 80^{\mathrm{WT}}$ control cells, both mutants had an increased number of metaphase cells containing 3-5 unaligned chromosomes. Cells rescued with the $\mathrm{Ndc} 80^{\Delta 60-80}$ mutant had a slightly reduced number of the metaphase aligned cells $(13.4 \pm 1.7 \%$ of mitotic cells $)$, but this reduction might have been a result of a portion of the mitotic cell population proceeding on to anaphase (see below). Finally, cells rescued with the N-terminal $\mathrm{Ndc} 80^{\Delta 1-40}$ mutant were able to align $11.0 \pm 1.7 \%$ of mitotic cells into a metaphase plate. These data suggest that both regions of the tail are required for optimal chromosome alignment, but the loss of the Nterminal half of the tail has a more severe effect on the congression.

The designed truncations not only removed charged residues and Aurora B phosphorylation sites, they also reduced tail length. To exclude the potential effects of a shorter tail length, we generated a pair of $\mathrm{Ndc} 80$ mutants that maintained a full-length tail but had a reduced net charge of +4 compared to +10 of the wild type tail. One mutant, $\mathrm{Ndc} 80^{+4 \mathrm{NT}}$, targeted the N-terminal region of the tail by mutating lysines 2, 26 and 35 and arginines 3, 13 and 20 to alanines thus destroying the consensus sequences for the Aurora sites S4, S5, and S15 in the process. The complementary mutant, Ndc $80^{+4 \mathrm{CT}}$, targeted the C-terminal region of the tail. Here, lysines 42, 47, 53, and 59 and arginines 52 and 60 were mutated to alanines, and the Aurora S44, T49, S55 and S62 sites were nullified (Figure 2.1.A). 
The Ndc $80^{+4 \mathrm{CT}}$ mutant was capable of aligning chromosomes $(12.0 \pm 1.7 \%$ of total mitotic cells). Strikingly, the Ndc80 $0^{+4 N T}$ mutant was far less effective at aligning chromosomes than the $\mathrm{Ndc} 80^{\Delta 1-40}$ mutant. Only $1.7 \pm 0.8 \%$ of mitotic Ndc80 $0^{+4 \mathrm{NT}}$ cells were able to align their chromosomes (Figure 2.1.B and C), making the Ndc80 ${ }^{+4 \mathrm{NT}}$ alignment phenotype more comparable to the phenotype achieved by the loss of the entire tail or the loss of all net positive charges from the tail. Therefore, reducing the net charge in either of the tail regions compromises chromosome alignment by kinetochores, although mutation in the $\mathrm{N}$-terminal region is more detrimental. Furthermore, this result reinforces the concept that the $\mathrm{Ndc} 80$ tail is not a random stretch of positive charges, but is rather composed of two distinct regions. It should be noted that the $\mathrm{Ndc} 80^{+4 \mathrm{NT}}$ and the $\mathrm{Ndc} 80^{+4 \mathrm{CT}}$ mutants have more severe alignment defects than the corresponding $\mathrm{N}$ - and $\mathrm{C}$ terminal truncation mutants. The reason for these discrepancies remains unknown.

Next, we measured the ability of kinetochores containing mutated Ndc80 protein to exert pulling forces on bound microtubules. We measured the distance between the sister kinetochores (inter-kinetochore distance) that are stretched apart by the pulling forces exerted by microtubules and serve as readouts for the strength of kinetochoremicrotubule interactions. Cells in early prometaphase have formed very few productive kinetochore-microtubule interactions, and inter-kinetochore distance measurements for these cells were therefore comparable to Ndc80 knockdown cells or cells treated with high doses of nocodazole (Figure 2.1.D). As few cells expressing the $\mathrm{Ndc} 80^{+4 \mathrm{NT}}$ mutant had metaphase-aligned chromosomes, we measured late prometaphase $\mathrm{Ndc} 80^{+4 \mathrm{NT}}$ cells instead. The average measurement for these cells was $1.00 \pm 0.02 \mu \mathrm{m}$, suggesting that kinetochores containing the $\mathrm{Ndc} 80^{+4 \mathrm{NT}}$ mutant generated reduced amount of force. In contrast, aligned chromosomes from $\mathrm{Ndc} 80^{+4 \mathrm{CT}}$ metaphase cells had an average interkinetochore distance measurement of $1.18 \pm 0.04 \mu \mathrm{m}$, which is similar to the average 
measurement for $\mathrm{Ndc} 80^{\mathrm{WT}}$ metaphase chromosomes $(1.23 \pm 0.02 \mu \mathrm{m})$. Metaphase $\mathrm{Ndc} 80^{\Delta 20-40}$ cells (average measurement of $\left.1.15 \pm 0.05 \mu \mathrm{m}\right)$ and $\mathrm{Ndc} 80^{\Delta 40-60}$ cells $(1.19 \pm$ $0.02 \mu \mathrm{m})$ also generated the wild type levels of stretch.

\section{$\mathrm{Ndc80}^{+4 \mathrm{CT}}$ arrests cells in metaphase due to both SAC and Aurora B activation}

Though both $\mathrm{Ndc} 80^{+4 \mathrm{CT}}$ and $\mathrm{Ndc} 80^{\Delta 40-60}$ cells were capable of aligning chromosomes to a metaphase plate and generating full tension on aligned chromosomes, not a single cell expressing either of these two mutants progressed into anaphase at the time of fixation. In contrast, on average $4.4 \pm 0.7 \%$ of $\mathrm{Ndc} 80^{\mathrm{WT}}$ mitotic cells and $8.0 \pm$ $4.6 \%$ of $\mathrm{Ndc} 80^{\triangle 60-80}$ mitotic cells were in anaphase (Figure 2.2.A). This suggests that impairing the function of the C-terminal microtubule binding region, either by engineering point mutations or making wholesale deletions, halts cell cycle progression.

To determine if this delay was caused by an active spindle checkpoint signal, we performed a knockdown and rescue experiment whereby we knocked down the spindle checkpoint protein Mad2 in conjunction with replacement of the Ndc80 mutant. Despite being fixed at the same time point as $\mathrm{Ndc} 80^{+4 \mathrm{CT}}$ cells, $28 \%$ of $\mathrm{Ndc} 80^{+4 \mathrm{CT}} / \mathrm{Mad} 2$ knockdown cells in mitosis were scored as anaphase (Figure 2.2.A). The failure of cells expressing $\mathrm{Ndc} 80^{+4 \mathrm{CT}}$ could be due to a failure to remove Mad2 from kinetochores following microtubule attachment, which is a phenotype seen for point mutants of the kinetochore component Spindly [186]. However, when we examined the kinetochore localization of Mad2 in metaphase $\mathrm{Ndc}^{+4} 0^{+4 \mathrm{CT}}$ cells, we found that Mad2 was lost from the kinetochore as effectively as in $\mathrm{Ndc} 80^{\mathrm{WT}}$ controls (Figure 2.2.D). Similarly, we found that BubR1 was capable of localizing to $\mathrm{Ndc} 80^{+4 \mathrm{CT}}$ kinetochores, as is seen for metaphase $\mathrm{Ndc} 80^{\mathrm{WT}}$ cells. Therefore, we conclude that the failure of our $\mathrm{Ndc} 80^{+4 \mathrm{CT}}$ mutant to progress into anaphase was due to active spindle checkpoint signaling from kinetochores with depleted levels of Mad2. 
We previously showed that there are two branches of the spindle checkpoint, one regulated by Aurora B and a second by CENP-I. To determine which branch was active in $\mathrm{Ndc} 80^{+4 \mathrm{CT}}$ expressing cells, we treated cells stably expressing $\mathrm{Ndc} 80^{\mathrm{WT}}$ or $\mathrm{Ndc} 80^{+4 \mathrm{CT}}$ with the Aurora B inhibitor ZM447439. At the time of fixation, $18.6 \pm 1.5 \%$ of DMSOtreated $\mathrm{Ndc} 80^{\mathrm{WT}}$ cells were in mitosis, compared to $38.7 \pm 11.0 \%$ of DMSO-treated $\mathrm{Ndc} 80^{+4 \mathrm{CT}}$ cells - again confirming the cell cycle arrest observed with this mutant. Following the treatment with the inhibitor, the mitotic index of $\mathrm{Ndc} 80^{\mathrm{WT}}$ cells $(16.2 \pm$ $1.6 \%$ of total cells in mitosis) was largely unchanged (Figure 2.2.B). In contrast, the mitotic index of ZM447439 treated Ndc80 ${ }^{+4 \mathrm{CT}}$ cells fell markedly to $24.9 \pm 1.8 \%$ of the total cell population. In contrast knock-down of CENP-I did not substantially change the mitotic index of either cell line (Figure 2.2.C). Therefore, cells expressing the Ndc $80^{+4 \mathrm{CT}}$ mutant arrest in an Aurora B dependent manner.

\section{C-terminal mutant of the Ndc80 tail retains high affinity to microtubules but lacks} ability to cluster in vitro

To test how $+4 \mathrm{CT}$ mutation in Ndc80 tail influences the binding of the complex to microtubules in vitro, we utilized a fluorescence anisotropy binding assay. We used an engineered version of the $\mathrm{Ndc} 80$ complex, known as $\mathrm{Ndc} 80^{\text {Bonsai }}$ [93], for in vitro analysis. Wild-type Ndc80 ${ }^{\text {Bonsai }}$ interacted with microtubules with measured $K_{d}$ of $222 \pm$ $65.4 \mathrm{nM}$ when fitted using the Hill equation, which is in agreement with previous reports [93]. The interaction between $\mathrm{Ndc} 80^{\text {Bonsai }+4 \mathrm{CT}}$ mutant and microtubules had slightly higher binding affinity as compared with $\mathrm{Ndc} 80^{\text {Bonsai }}$ WT, with a $\mathrm{K}_{\mathrm{d}}$ of $131.8 \pm 55.2 \mathrm{nM}$ (Figure 2.3.A). This distinguishes $+4 \mathrm{CT}$ mutant from phospho-mimetic mutants in that region that show reduced affinity instead. It also supports the notion that $\mathrm{Ndc} 80^{+4 \mathrm{CT}}$ cells are capable of forming proper kinetochore-microtubule attachments. Strikingly, the Hill coefficient was noticeably reduced between $\mathrm{Ndc}^{\mathrm{B}} \mathrm{B}^{\mathrm{Bnsai}} \mathrm{WT}^{\mathrm{W}}(\mathrm{h}=0.842 \pm 0.229)$ and 
Ndc $80^{\text {Bonsai }}+4 \mathrm{CT}(\mathrm{h}=0.415 \pm 0.148)$. It suggests a negative cooperativity of the interaction between $\mathrm{Ndc} 80^{\text {Bonsai }+4 \mathrm{CT}}$ and the microtubules.

Structural studies suggested that the Ndc80 tail contributes to cooperativity of the interaction with microtubules. In addition $\mathrm{Ndc} 80^{\text {Bonsai }}$ molecules cluster along microtubule protofilaments $[108,117]$. To determine if $\mathrm{Ndc} 80^{\text {Bonsai+4CT }}$ is deficient in the clustering on the microtubules, we used a negative stain electron tomography. Considering similar $\mathrm{K}_{\mathrm{d}}$ of interaction between $\mathrm{Ndc} 80^{\text {Bonsai }}$ WT and $+4 \mathrm{CT}$ with microtubules but different values of Hill coefficient, we speculated that the main difference between the mutants should be in the distribution of the molecules along the microtubule filaments. As expected, we observed that the ability of Ndc $80^{\text {Bonsai }+4 \mathrm{CT}}$ mutant to form the clusters was impaired, as compared to $\mathrm{Ndc} 80^{\text {Bonsai WT }}$ (Figure 2.3.B, C).

\section{C-terminal region of $\mathrm{Ndc80}$ tail is required for $\mathrm{Ska3}$ recruitment to the kinetochore}

To determine the cause of the tension defect sensed by the checkpoint, we turned our focus to a pair of kinetochore components that play a role in the still-mysterious process of microtubule attachment "maturation", and whose loss delays the metaphase-toanaphase transition in cells. The first protein, Small Kinetochore Associated Protein (SKAP), is a microtubule-associated protein involved in recruitment of astrin to microtubule plus ends. Loss of either SKAP or astrin results in delays in the chromosome alignment [221]. The loss of the second component, the Ska complex, results in cells that can align chromosomes into a metaphase plate but fail to satisfy the signal checkpoint and do not enter anaphase [137]. We examined the kinetochore localization of SKAP and Ska3 in $\mathrm{Ndc}^{\mathrm{WT}}$ and $\mathrm{Ndc} 80^{+4 \mathrm{CT}}$ cells. Both SKAP and Ska3 were present at the kinetochores of metaphase $\mathrm{Ndc} 80^{\mathrm{WT}}$ cells. In $\mathrm{Ndc} 80^{+4 \mathrm{CT}}$ cells however, Ska3 was absent 
from the kinetochores (Figure 2.4.A, B). These data suggest that the failure to recruit the Ska complex to kinetochores causes $\mathrm{Ndc} 80^{+4 \mathrm{CT}}$ cells to arrest in mitosis.

\section{Ska3 recruitment to the kinetochore is dependent on Aurora B phosphorylation of}

\section{Ndc80 tail.}

Ska complex localizes to kinetochores in prometaphase, and is dependent on microtubule occupancy at the kinetochores [133,135]. Ska levels at the kinetochores increase about twofold in metaphase, as compared to prometaphase, and is downregulated by Aurora B-mediated Ska1 and Ska3 phosphorylation. While Ska3 kinetochore levels are significantly reduced in the absence of microtubules in nocodazole treated cells, inhibition of Aurora B restores Ska complex localization to kinetochores. This phenotype is currently attributed to the inhibiting role of Aurora B mediated Ska phosphorylation [139]. Although it was shown that knock-down of various proteins such as Shugoshin, Ndc80, Mis12 and Knl1 blocks the Ska complex localization to the kinetochore, it is still unclear which component is directly responsible for Ska recruitment [137,139]. To obtain insight into the mechanism of the Ska complex localization to the kinetochore in the absence of the microtubules, we treated $\mathrm{Ndc} 80^{+4 \mathrm{CT}}$ cells with nocodazole and Aurora B inhibitor, ZM447439 [222]. Ska3 kinetochore levels were significantly lower in $\mathrm{Ndc} 80^{+4 \mathrm{CT}}$ cells, as compared to $\mathrm{Ndc} 80^{\mathrm{WT}}$ (Figure 2.4.C and D). This strongly suggests that the Ndc80 tail is required not only to form proper attachments to the microtubules, but is also required for Ska recruitment to the kinetochore.

The inability of the $\mathrm{Ndc} 80^{+4 \mathrm{CT}}$ tail mutant to localize the Ska complex to the kinetochore may indicate a direct interaction between the two wild-type proteins instead. Although we failed to confirm such interaction, we observed an increase of the PP1 activity on the Ndc80 ${ }^{\text {Bonsai }}$ phosphosubstrate in presence of Ska (Figure 2.4.E). We also 
noticed an increase in the binding affinity of Ska to microtubules in vitro when Ndc80 was present (data not shown). We note that our assay was performed with Ndc80 ${ }^{\text {Bonsai }}$ and Xenopus Ska complex demonstrating that the interaction does not require the coiled coil or loop region that were present in the previous work [110].

Because we were unable to observe a direct interaction between Ska and Ndc80 in vitro we utilized Proximity Ligation Assay (PLA, [223]), which measures the close proximity of two antigens in an immunofluorescence like experiment in vivo. PLA was used to measure proximity between the Ndc80 Calponin homology domain (monoclonal 9G3) and a polyclonal antibody against the Ska3 protein. After the PLA reaction was performed, the cells were additionally stained with antibodies to tubulin and Borealin to identify the spindle and inner-centromeres respectively. The PLA signal was highly enriched at centromeres of mitotic cells and these signals were rarely found in control cells that lacked one of the two primary antibodies in the PLA reaction (Figure 2.5.A). There were also a few foci of PLA signal in the cytoplasm of mitotic cells that may represent interactions between soluble complexes. We quantified the number of Ndc80Ska3 PLA spots adjacent to centromeres and Ndc80 and Ska3 appear to be in close proximity in both prometaphase and metaphase centromeres (Figure 2.5.B). 67\% of prometaphase centromeres were associated at least one PLA signal and this increased to $80 \%$ of centromeres in metaphase. The number of centromeres associated with two PLA signals facing opposite poles increased in metaphase relative to prometaphase but the difference was not significant (Figure 2.5.C). These data suggest that Ndc80 and Ska reside in close proximity at end-on attached kinetochores, which is consistent with the Ndc80 CHD or tail binding Ska in vivo. 


\section{Ska complex forms V-shaped structures on microtubules in presence of Ndc80}

The Ska complex was previously shown to stimulate the association of the Ndc80 complex with microtubules. Additionally, presence of Ska allows Ndc80 to track depolymerizing microtubule plus-ends [110]. This feature is likely to contribute to stability of kinetochore-microtubule attachments in vivo. Although the mechanism is still unknown, it is likely that Ska facilitates tracking of Ndc80 molecules by forming a complex on the surface of the microtubule. To investigate this possibility we used negative stain electron tomography and analyzed the mixture of Ndc80 ${ }^{\text {Bonsai }}$ WT and Ska bound to taxol-stabilized microtubules. We identified unique V-shaped structures on the microtubule that requires both $\mathrm{Ndc} 80$ and Ska to form (Figure 2.6.A). The core of the Ska complex forms an assembly that is similar in both shape and size (180 $\mathrm{A}$ x $80 \AA$ [144], note the lack of Ska1 CTD and Ska3 C-terminal region in this structure) as those novel structures $(227 \pm 28 \AA$ x $85 \pm 7 \AA$, ten V-shapes measured). Additionally, we observed similar structures on microtubules incubated with $\mathrm{Ndc} 80^{\text {Bonsai }}$ WT and Xenopus Ska complex that were not observed in absence of Ska. Moreover, we found Ndc80 cluster in the same position on the microtubules in layers underneath some of the V-shapes (Figure 2.6.B). We suspect that the $\mathrm{V}$-shapes are formed only on $\mathrm{Ndc} 80$ oligomeric assemblies as we did not observe any in absence of Ndc80 or in presence of Ndc $80^{\text {Bonsai }}+4 \mathrm{CT}$ (data not shown). Therefore we hypothesize that the $\mathrm{V}$-shapes observed in our tomographic reconstructions are Ska molecules interacting with the Ndc80 clusters. 


\section{$\underline{\text { Discussion }}$}

Insight into "end-on" attachment by the kinetochore and depolymerization-coupled movement

The Ndc80 complex makes "end-on" microtubule attachments to the kinetochore, required for kinetochores to utilize microtubule depolymerization to generate chromosomal movements to the metaphase plate and segregation of chromatids to the poles in anaphase. Current models suggest that the Ndc80 complex utilizes multiple weak attachment points to bind the lateral outer surface of a microtubule. These attachments are lost when the protofilaments begin to curve during depolymerization generating a tight attachment that is exquisitely sensitive to the conformation of the microtubule. The Ndc80 complex utilizes a four-part microtubule attachment point to interact with the microtubule polymer. First, the "toe" region of the CHD of the Ndc80 subunit directly binds the surface of microtubules between adjacent tubulin subunits. A second positively charged region of the CHD interacts with the E-hooks on the C-terminus of tubulin. Third, when the Ndc80 complex is not adjacent to another Ndc80 complex the unstructured tail has a direct microtubule attachment point. Fourth, after alignment along a protofilament the unstructured tails both mediate this alignment by binding between adjacent subunits and they form a domain that can also interact with a second E-hook on an adjacent protofilament. Mutation of either of the first two-microtubule attachment points completely eliminates cold stable microtubule attachments and the depolymerization-coupled movement that aligns chromosomes to the metaphase plate. Elimination of the entire tail also eliminates binding but whether microtubule attachment by the tail is the reason for this failure is not known. A second outstanding question is whether the Ndc80 ever align along protofilaments as recent studies argue that multiple 
Ndc80 proteins act alone on a microtubule and do not need to align along protofilaments [120].

Our structure-function analysis of the unstructured Ndc80 tail provides insight into both of these questions. We showed that the ability to form proper microtubule attachments and align chromosomes is retained even when the first 60 amino acids of the tail were removed, leaving only 20 amino acids and the net charge of +1 . This important experiment argues for a reexamination of the tail since the major function is carried out with a net charge of +1 , no Aurora B sites and no known microtubule binding affinity. The phosphorylation of the tail by the Aurora kinase regulates the binding to microtubules in a rheostat-like manner [120]. The Ndc80 tail contains a net charge of +10 , suggesting the binding with negatively charged microtubule surface occurs through ionic interactions. In agreement with this model we find that elimination of the charge or just removal of the charge in the N-terminal 40 amino acids eliminates the binding of kinetochores to microtubules, however, our data also argue for greater complexity of the role of the tail region. By altering the net charge of the C-terminal region of the tail, we were able to create a mutant $\left(\mathrm{Ndc} 80^{+4 \mathrm{CT}}\right)$ capable of forming microtubule attachments and aligning the chromosomes to the metaphase plate, although it was still unable to progress to anaphase in a SAC-dependent manner. This mutant can still bind the microtubules with similar, even stronger, affinity as compared to the wild-type. However, it fails to form larger clusters on the microtubule lattice in vitro, which is consistent with reduced cooperativity, as measured by a negative Hill coefficient in equilibrium binding assays. Thus the $+4 \mathrm{CT}$ mutant is interesting as it probably reduces the ability of Ndc80 to align along protofilaments. We find that cells expressing the $+4 \mathrm{CT}$ mutant are able to bind microtubules, align chromosomes to the metaphase plate and generate full interkinetochore tension. Thus our mutant provides the first in vivo evidence that $\mathrm{Ndc} 80$ 
do not need to cluster along protofilaments to carry out most of its functions as previously postulated from in silico and in vitro characterization.

Insight into the final stages of "end-on" attachments and silencing of the spindle checkpoint.

Cells expressing the +4CT mutant arrest in metaphase, which suggests that clustering of Ndc80 molecules along protofilaments has an unrecognized role in silencing the SAC. Interestingly, this mutant cannot recruit the Ska complex to the kinetochore. In collaboration with Gary Gorbsky and Hongtao Yu, we have recently shown that Ska recruits PP1 to the kinetochore to enable the progression to anaphase (See Chapter III and [220]). Thus, our data demonstrate that the tail of Ndc80 has a role in recruiting the Ska complex. Ska and Ndc80 have been shown to directly bind on microtubules and Ndc80 and Ska localize in close proximity to the kinetochore as measured by PLA. Thus this may be a direct interaction. Identification of novel structures by negative stain tomographic reconstruction of microtubules decorated by Ska and Ndc80 is a discovery that indicates that indeed Ska and Ndc80 directly interact on microtubules. Those Vshaped structures, similar in shape and size to the structure of the Ska core complex, were observed exclusively in the presence of both Ndc80 and Ska, and only when wild-type Ndc80 was used. Additionally we show that Ndc80 clusters are positioned directly underneath the V-shapes. Consistent with other results discussed before, it suggests that the clustering of Ndc80 molecules is required for the interaction between the complexes on the microtubules. The simplest model is that Ska recognizes Ndc80 CHDs clustered along a protofilaments, e.g. through the electrostatic interaction between negatively charged patches on the Ska core complex [144] and positively charged patches on the surface of the Ndc80 CHD [93] or the N-terminal tail. However, it is also possible that 
the binding to the microtubules causes structural rearrangement of the Ndc80 tail so it becomes recognizable by Ska.

How Ska is recruited to the kinetochore has been an enigma. Ska localizes poorly to kinetochores in cells treated with nocodazole, has intermediate binding in prometaphase and its levels are highest at metaphase. Ska recognition of either clustered Ndc 80 or of the region of the tail that is exposed when Ndc80 binds with microtubules can explain these properties. However, Ska can also localize to kinetochores in cells treated with nocodazole and Aurora inhibitors. Aurora can directly phosphorylate Ska and inhibit its recruitment, but it is also possible that Aurora phosphorylation of the Ndc80 tail prevents clustering of adjacent Ndc80 proteins not bound to microtubules which could be recognized by Ska (Figure 2.8).

In conclusion, the tail of $\mathrm{Ndc} 80$ has been largely thought of as regulator of $\mathrm{Ndc} 80$ binding to microtubules, acting by decreasing the $\mathrm{k}_{\text {off }}$ and this activity is countered by Aurora phosphorylation. Our studies suggest that the tail has additional functions in recruiting Ska and that the clustering of Ndc80 along protofilaments may be the key event to recruit Ska/PP1 and drive mitotic exit. 


\section{Material and Methods}

\section{Cloning of $\mathrm{Ndc80}$ constructs}

$\mathrm{Ndc} 80^{+4 \mathrm{NT}}$ and $\mathrm{Ndc} 80^{+4 \mathrm{CT}}$ mutants were generated by two-step PCR mutagenesis using the $\mathrm{Ndc}^{+0} 0^{+0}$ construct $\left(\mathrm{Ndc} 80^{\mathrm{NEU}}\right)$ [107] as a template. $\mathrm{Ndc} 80^{+4 \mathrm{NT}}$ contains the following 6 Ndc80 tail residues mutated to alanine: K2, R3, R13, R20, K26, and K35. $\mathrm{Ndc} 80^{+4 \mathrm{CT}}$ contains the following $6 \mathrm{Ndc} 80$ tail residues mutated to alanine: K42, K47, R52, K53, K59, and R60. The Ndc80 truncation mutants were generated by Splice Overlap Extension (SOE) PCR mutagenesis using the Ndc80 ${ }^{\mathrm{WT}}$ construct (Tooley et. al., 2011) as a template. Sequence corresponding to N-terminal region of Ndc $80^{\text {Bonsai }} \mathrm{WT}$ and +4CT mutant was codon optimized, synthesized (GENEART) and inserted to the Ndc80 Bonsai construct as described in [108]. All constructs generated were verified by sequencing (Genewiz).

\section{Protein purification and negative stain sample preparation}

6xHis-PP1 $\alpha$ 7-330 was expressed and purified essentially as previously described [224] with following modifications. Prior to elution, Ni-NTA IMAC (Qiagen) was incubated with wash buffer supplemented with $5 \mathrm{mM}$ ATP and $5 \mathrm{mM} \mathrm{MgCl} 2$ for $2 \mathrm{hr}$ and subsequently washed with wash buffer. PP1 was further purified on Superdex 200 10/300 GL size-exclusion column in $50 \mathrm{mM}$ HEPES pH 7.5, $150 \mathrm{mM} \mathrm{KCl,} 1 \mathrm{mM}$ DTT.

7xHis-Ska2/Ska1 (a kind gift from Iain M Cheeseman) was expressed in BL21 (DE3) cells for $4 \mathrm{hrs}$ at $20^{\circ} \mathrm{C}$, purified on NiNTA resin following standard protocol, and subjected to size exclusion on Superdex 75 10/300 GL column. For fluorescence anisotropy experiments $\mathrm{Ndc} 80^{\text {Bonsai }} \mathrm{WT}$ and $+4 \mathrm{CT}$ mutant were purified similarly as described previously [93]. For electron tomography experiments following modifications were applied. After incubation of the cell lysate with the Glutathione Sepharose 4 Fast 
Flow (Sigma-Aldrich) resin proteins were washed and eluted with buffer containing 10mM reduced glutathione and subjected to gel filtration on Superdex 200 10/300 GL size-exclusion column, re-bound to glutathione resin and washed into BRB80 + $1 \mathrm{mM}$ DTT $+0.05 \%$ NP-40. Untagged Ndc $80^{\text {Bonsai }}$ was eluted by GST-tag cleavage with HRV 3C Protease.

Preparation of negatively stained samples was done essentially as previously described [117]. For experiment depicted in Figure 2.6.A EM grids were sequentially incubated with $1 \mu \mathrm{M}$ taxol-stabilized microtubules for 30 seconds, $1 \mu \mathrm{M}$ Ndc $80^{\text {Bonsai WT }}$ for $3 \mathrm{~min}$ and $0.5 \mu \mathrm{M}$ of the Ska complex for $2 \mathrm{~min}$, followed by staining by $2 \%$ uranyl formate. Tilt series were manually collected from -60 to 60 degrees with Tecnai F20 operating on $120 \mathrm{kV}$, at nominal magnification of 52,000x and $1.2 \mu \mathrm{m}$ underfocus. IMOD software package was used for tomographic reconstructions [225].

\section{Knockdown and rescue of $\mathrm{Ndc80}$ in transiently transfected HeLa cells}

HeLa cells (ATCC) were maintained in Dulbecco's modified Eagle's medium (Invitrogen) supplemented with $10 \%$ fetal bovine serum (Invitrogen) in a humidified incubator at $37^{\circ} \mathrm{C}$ with $5 \% \mathrm{CO}_{2}$. For synchronization, cells were seeded in media containing $2 \mathrm{mM}$ thymidine for $24 \mathrm{hr}$, released into fresh media for $12 \mathrm{hr}$, arrested again in $2 \mathrm{mM}$ thymidine for $12 \mathrm{hr}$, released for $8-12 \mathrm{hr}$, and fixed for immunofluorescence. Ndc80 siRNA sequence and rescue sequence modifications were used as previously described [105,107]. For knockdown and replacement experiments, cells were cotransfected at the first thymidine release with siRNA oligos $(75 \mu \mathrm{M}$ for Ndc80, $20 \mu \mathrm{M}$ for Mad2) and $100 \mathrm{ng}$ rescue plasmid using Lipofectamine 2000 (Invitrogen). Cells were transfected a second time with siRNA oligos at the second thymidine block using RNAiMax (Invitrogen). For mock and siRNA only controls, an empty pEGFP vector was 
used as the rescue plasmid. For transient transfection Ndc80 tail experiments, $75 \mu \mathrm{M}$ GAPD siRNA oligos (Thermo Scientific) were included as mock controls.

\section{Creation of stable cell line}

To create a stable cell line expressing the $\mathrm{Ndc} 80^{\mathrm{WT}}$ and $\mathrm{Ndc} 80^{+4 \mathrm{CT}}$ tail mutant, Ndc $80^{+4 \mathrm{CT}}$-GFP was cloned into the pCDNA5/FRT plasmid (Invitrogen) using flanking Not1 restriction sites. The plasmid was co-transfected into T-Rex HeLa cells (Invitrogen) with the pOG44 plasmid (Invitrogen) and the cells were cultured in DMEM $+5 \%$ FBS (GIBCO) supplemented with hygromycin B (Invitrogen) for 14 days. At the end of the selection period, remaining cells were pooled and used for subsequent experiments.

\section{Aurora inhibition and CENP-I knockdown experiments}

For Aurora inhibition experiments, HeLa cell lines stably expressing Ndc80 ${ }^{\mathrm{WT}}$ and $\mathrm{Ndc} 80^{+4 \mathrm{CT}}$ were treated with thymidine and siRNA oligos similar to transient transfection knockdown and rescue experiments, with the following exception: Lipofectamine RNAiMax (Invitrogen) was used in place of Lipofectamine 2000 (Invitrogen) at the first siRNA transfection. One hour prior to fixation ( $\sim 36$ hours from start of experiment), cells were treated with $3.3 \mu \mathrm{M}$ nocodazole (Sigma-Aldrich) and/or 2 $\mu \mathrm{M}$ ZM447439 (Tocris) for one hour. For CENP-I knockdown experiments in HeLa cell lines stably expressing $\mathrm{Ndc} 80^{\mathrm{WT}}$ and $\mathrm{Ndc} 80^{+4 \mathrm{CT}}$, either $20 \mathrm{nM}$ CENP-I ON-Target SmartPool siRNA (Dharmacon) or 20nM LacZ control siRNA (Dharmacon) were cotransfected with $75 \mathrm{nM}$ Ndc80 siRNA using the RNAiMax transfection reagent. Experiment timeline and other conditions used for transient knockdown and rescue experiments were maintained. 


\section{Immunofluorescence}

For most of the immunofluorescence experiments, coverslips were co-fixed and extracted in PHEM buffer containing 2\% paraformaldehyde and 0.5\% Triton X-100 for 20 min at room temperature. Ska3 and SKAP coverslips were fixed in ice-cold methanol for 10 minutes. Antibodies used were anti-Ndc80 9G3 (1:500 [vol/vol]; GTX70268, GeneTex), anti-GFP (1:500 [vol/vol]), anti-ACA (1:100 [vol/vol], Antibodies Incorporated), anti-tubulin (1:500 [vol/vol], DM1 $\alpha$, NeoMarkers), anti-BubR1 (1:500 [vol/vol]), anti-Mad2 (1:100 [vol/vol], a kind gift from Gary Gorbsky), anti-Ska3 (1.5 $\mu \mathrm{g} / \mathrm{mL}$, a kind gift from Gary Gorbsky), anti-SKAP (1.5 $\mu \mathrm{g} / \mathrm{mL}$, a kind gift from Gary Gorbsky) and FITC conjugated anti-tubulin (1:500 [vol/vol] DM1 $\alpha$, Sigma). DAPI staining (1:5000 of a $5 \mathrm{mg} / \mathrm{ml}$ stock) was used to visualize. The Ska3 and SKAP images from Figure 4B were collected using a 100x lens on a Deltavision microscope (Applied Precision) and deconvoluted z-projections are shown. Remaining images were captured as described previously [105] with Volocity 5.5 imaging software (Perkin Elmer). Quantification of the fluorescence intensities was done similarly to previously described [226]. Inter-kinetochore distance measurements were quantified using softWoRX imaging software.

\section{Proximity Ligation Assay}

Assay was performed as described previously [226]. Quantification of PLApositive centromeres was done in Volocity 6.3 software (PerkinElmer) in 3D Opacity mode, by semi-semi-automatic detection of the centromeric volumes and manual scoring of the surrounding PLA signals. 


\section{Phosphatase Activity Assay}

GST-Ndc $80^{\text {Bonsai }}$ WT was phosphorylated by Aurora A similarly to previously described method [226] and bound to Glutathione Sepharose 4 Fast Flow resin. Kinase and unreacted $\left[{ }^{32} \mathrm{P}\right] \mathrm{ATP}$ was subsequently removed by rigorous washes and untagged Ndc80 phosphosubstrate was removed from the resin by cleavage of GST-tag with HRV 3C Protease. Phosphatase activity assays were performed on $200 \mathrm{nM}$ Ndc80 phosphosubstrate, in phosphatase buffer (PBS, $1 \mathrm{mM} \mathrm{MnCl}_{2}, 1 \mathrm{mM}$ DTT) with $50 \mathrm{nM}$ hPP1 $\alpha$ 7-330 and increasing concentrations of 7xHis-Ska2/Ska1. Over the time-course of the experiment $1 \mu \mathrm{L}$ samples were transferred to $1 \mathrm{~mL}$ of $30 \%$ activated charcoal in 50 mM Na-phosphate buffer, $\mathrm{pH} 7.0$ and centrifuged. $500 \mu \mathrm{L}$ of the supernatant was mixed with $2 \mathrm{~mL}$ of scintillator and quantified in liquid scintillation counter. Initial rates of ${ }^{32} \mathrm{P}$ release were determined from the measurements of the initial 2 min of the reaction.

\section{Fluorescence Anisotropy}

Ndc $80^{\text {Bonsai }}$ WT and Ndc80 $0^{\text {Bonsai }}+4 \mathrm{CT}$ were labeled with Oregon Green 488 maleimide (ThermoFisher Scientific) according to manufacturer instructions and the unreacted dye was removed by size exclusion chromatography. Efficiency of labeling was estimated to be between 1.5 to 2 dye molecules per $\mathrm{Ndc} 80^{\text {Bonsai }}$ molecule. Increasing concentrations of taxol stabilized MT were incubated overnight with $50 \mathrm{nM}$ fluorescently labeled $\mathrm{Ndc} 80^{\text {Bonsai }}$, then $35 \mu \mathrm{L}$ of mixture was loaded in triplicates into 384 -well plate (Greiner Bio-One) and fluorescence anisotropy measurements were done in PHERAstar FS plate reader (BMG Labtech). The anisotropy data were fitted using Hill equation by OriginPro 7.5 software (Built-in non-linear fitting function Hill1, y $=$ Start $+($ End - Start $)$ $\left.* \mathrm{x}^{\mathrm{n}} /\left(\mathrm{k}^{\mathrm{n}}+\mathrm{x}^{\mathrm{n}}\right)\right)$. 


\section{Figures}

\section{Figure 2.1 Characterization of Ndc80 tail mutants}

(A) Cartoon depicting the wild type $\mathrm{Ndc80}$ tail and $\mathrm{Ndc80}$ tail mutants used for knockdown and rescue experiments in HeLa cells.

(B) Representative images of the predominant mitotic figures from siNdc80 knockdown and rescued cells that have been stained for Ndc80 (green), tubulin (cyan) and ACA (red). WT and Ndc80 tail mutant cells are identified by an expressed EGFP, and are costained for tubulin and DNA. White bar $=5 \mu \mathrm{m}$.

(C) Mitotic cells were scored for chromosome alignment into a metaphase plate, and the percentage of metaphase cells was plotted. Metaphase cells were further subdivided to indicate cells with all chromosomes aligned (black), cells with 1-2 unaligned chromosomes (gray) and cells with 3-5 unaligned chromosomes (white). >100 mitotic cells counted per experiment, $\mathrm{N}=3$. Error bars $=\mathrm{SD}$.

(D) Ten sister kinetochores in at least five cells $(\mathrm{N}>50)$ were identified by ACA staining between Ndc80 signals, and the distance between those sister kinetochores was measured $(\mathrm{N}=3)$. The mean distance is plotted for early prometaphase (EPM) (gray), late prometaphase (LPM) (dark gray), and metaphase (black) cells, Noc $=$ nocodazole-treated cells (white). Error bars $=$ SD. 


\section{Figure 2.1}

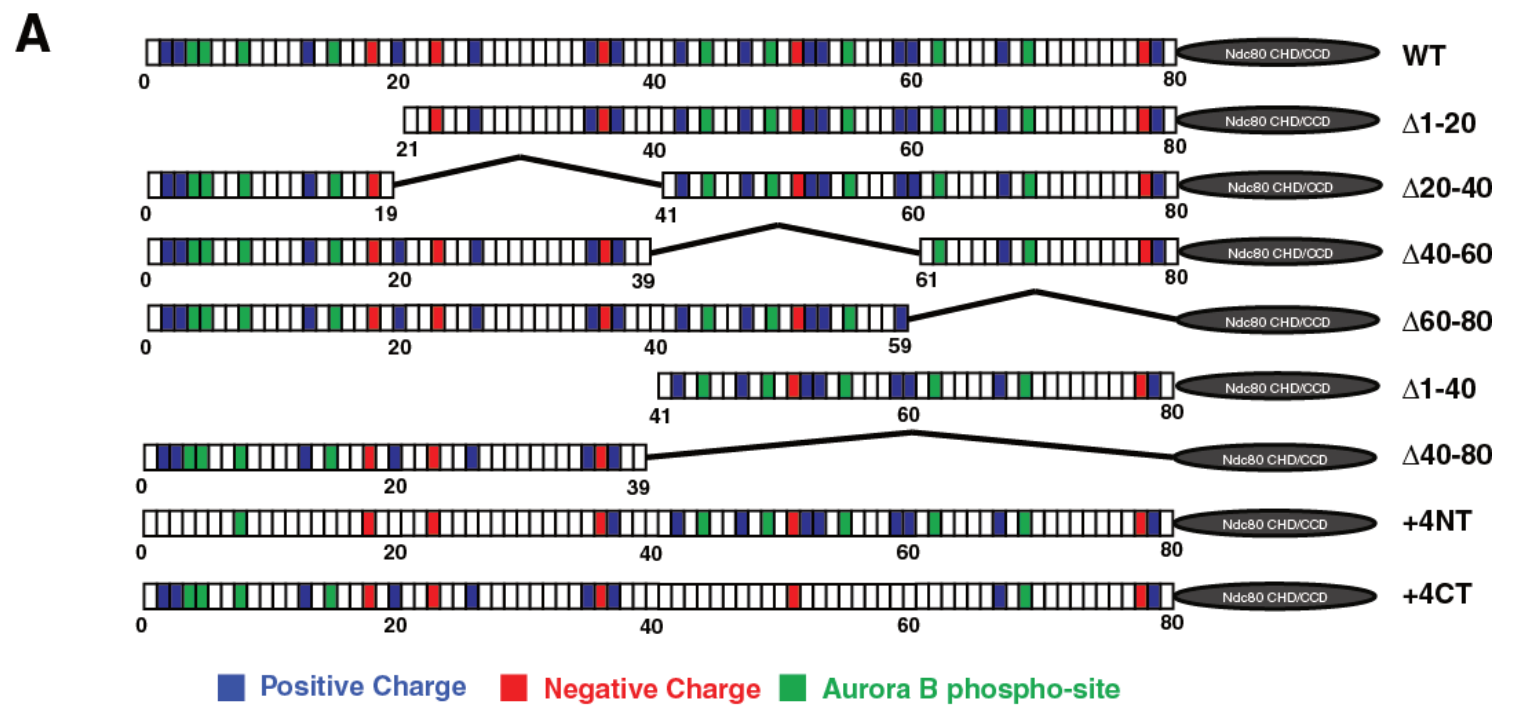

B

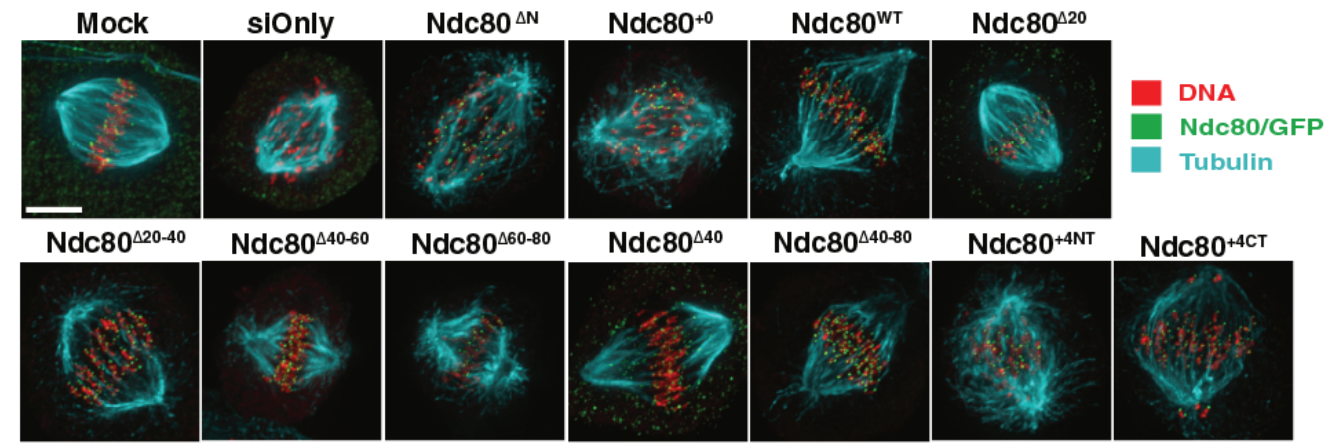

C

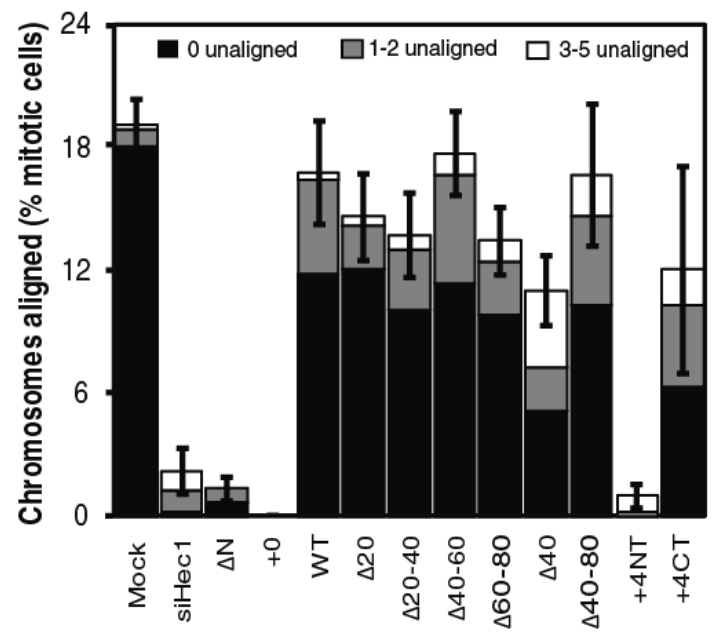

D

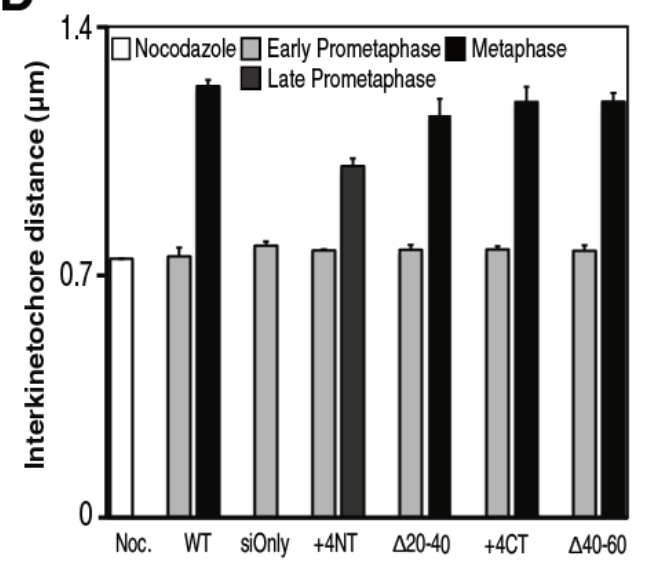

John Tooley and Pawel Janczyk 
Figure $2.2 \mathrm{Ndc80}^{+4 \mathrm{CT}}$ tail mutant metaphase arrest does not satisfy spindle assembly checkpoint and is Aurora dependent

(A) The percentage of mitotic cells in anaphase was plotted for $\mathrm{Ndc} 80^{\mathrm{WT}}$, $\mathrm{Ndc} 80$ knockdown and indicated Ndc80 tail mutant cells. For the $\mathrm{Ndc} 80^{+4 \mathrm{NT}}$ and $\mathrm{Ndc} 80^{+4 \mathrm{CT}}$ mutants, cells were also co-transfected with siRNA targeting Mad2. For Ndc80 tail mutants, one hundred mitotic cells counted per experiment $(\mathrm{N}=3)$. For Mad2 cotransfection with $\mathrm{Ndc} 80^{+4 \mathrm{CT}}$, twenty-five cells were counted. Error bars $=\mathrm{SD}$.

(B) $\mathrm{Ndc} 80^{+4 \mathrm{CT}}$ cells arrest in an Aurora dependent manner. $\mathrm{Ndc} 80^{\mathrm{WT}}$ and $\mathrm{Ndc} 80^{+4 \mathrm{CT}}$ stable HeLa cell lines were treated with $2 \mu \mathrm{M}$ ZM447439 for 1 hour prior to fixation at the conclusion of the knockdown and rescue protocol. The percentage of total cells in mitosis was counted and plotted. More than 200 total cells counted for each experiment, $\mathrm{N}=3$. Green bars $=$ DMSO control, orange bars $=2 \mu \mathrm{M}$ ZM447439. Error bars $=$ SD.

(C) Depletion of CENP-I does not reduce the mitotic index of $\mathrm{Ndc} 80^{+4 \mathrm{CT}}$ cells. $\mathrm{Ndc} 80^{\mathrm{WT}}$ and $\mathrm{Ndc} 80^{+4 \mathrm{CT}}$ stable HeLa cell lines were treated with two transfections of $20 \mathrm{nM} \mathrm{LacZ}$ or CENP-I siRNA prior to fixation at the conclusion of the knockdown and rescue protocol. The percentage of total cells in mitosis was counted and plotted. More than 200 total cells counted for each experiment, $\mathrm{N}=3$. Green bars = LacZ siRNA control, orange bars $=$ CENP-I siRNA. Error bars $=$ SD.

(D) Immunofluorescence staining of $\mathrm{Ndc} 80^{\mathrm{WT}}$ and $\mathrm{Ndc} 80^{+4 \mathrm{CT}}$ (green) stable lines after depletion of endogenous $\mathrm{Ndc} 80 . \mathrm{Ndc} 80^{+4 \mathrm{CT}}$ cells arrest with at least one $\mathrm{Mad} 2$ positive kinetochore (red, upper row) with no loss of BubR1 localization (red, lower row). White bar $=5 \mu \mathrm{m}$ 


\section{Figure 2.2}

A

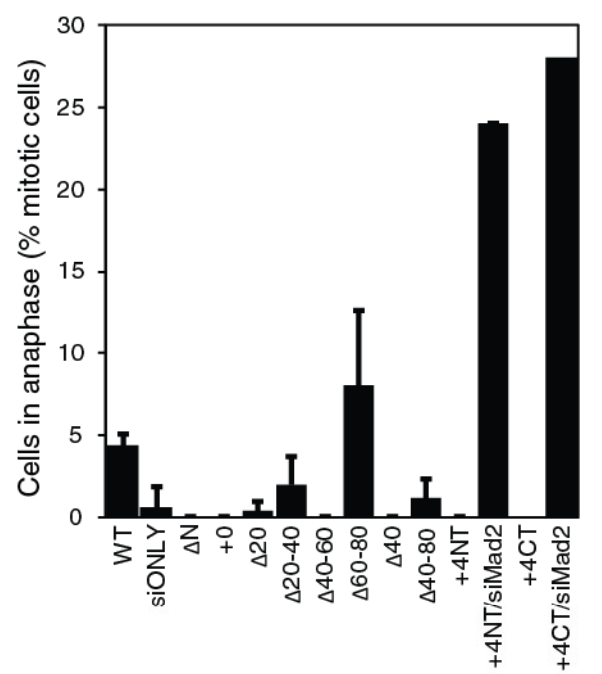

D

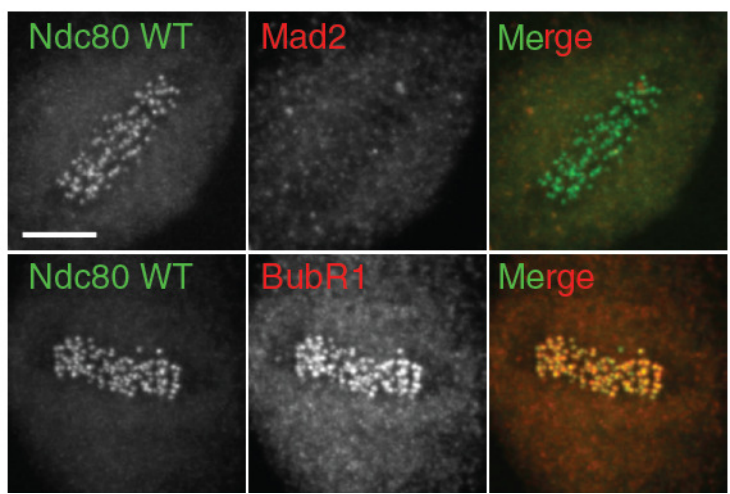

B

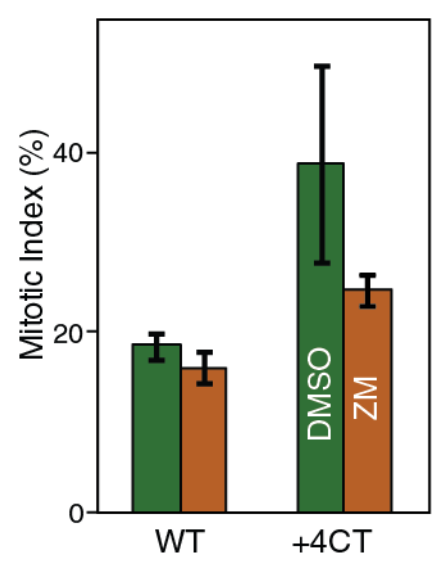

C

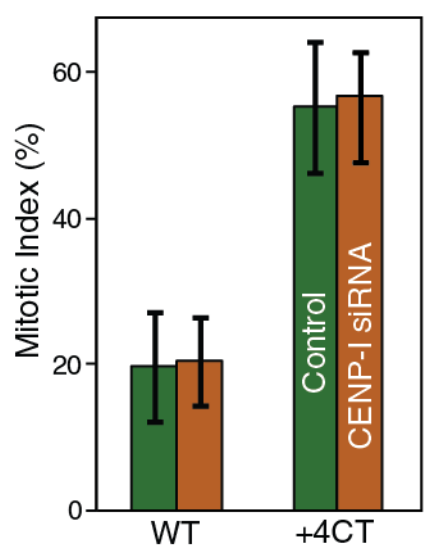

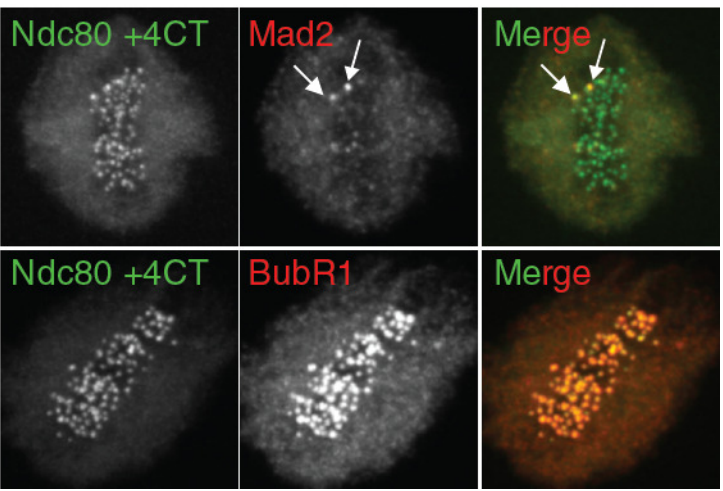

(A): John Tooley and Pawel Janczyk; $(B, C)$ : Daniel Matson; (D): Daniel Matson and Pawel Janczyk 


\section{Figure 2.3 Ndc80 +4CT tail mutant is deficient in clustering on microtubules.}

(A) Fluorescence anisotropy measurements of Oregon Green 488 covalently linked with $\mathrm{Ndc} 80^{\text {Bonsai }}$ WT (left panel, orange) or $\mathrm{Ndc} 80^{\text {Bonsai }}+4 \mathrm{CT}$ (right panel, dark green) incubated with increasing concentrations of taxol-stabilized microtubules, plotted on $\log _{10}$ scale. Hill equation was used for fitment of the data. Small graphs represent the data in the linear scale.

(B) Representative projections of 5 consecutive Z-sections of the tomographic reconstructions show $\mathrm{Ndc} 80^{\text {Bonsai }}+4 \mathrm{CT}$ form smaller clusters than $\mathrm{Ndc} 80^{\text {Bonsai }}$ WT. Black lines indicate the positions of the Ndc80 molecules.

(C) Quantification of cluster sizes represented in (B). N, number of quantified microtubules, $\mathrm{n}$, total number of $\mathrm{Ndc} 80^{\text {Bonsai }}$ molecules quantified. 


\section{Figure 2.3}

A
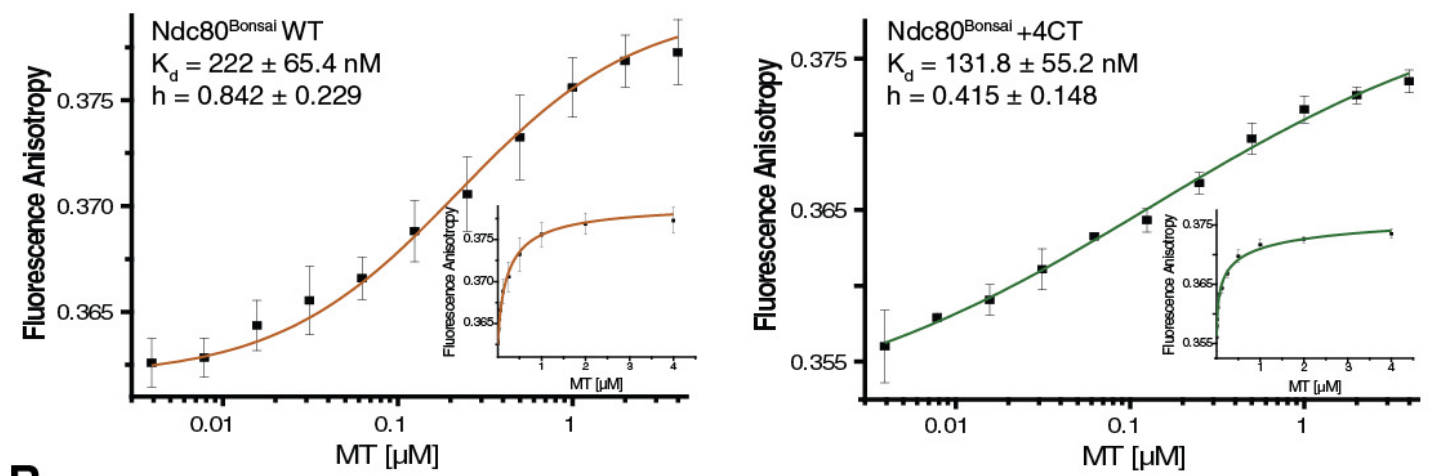

B
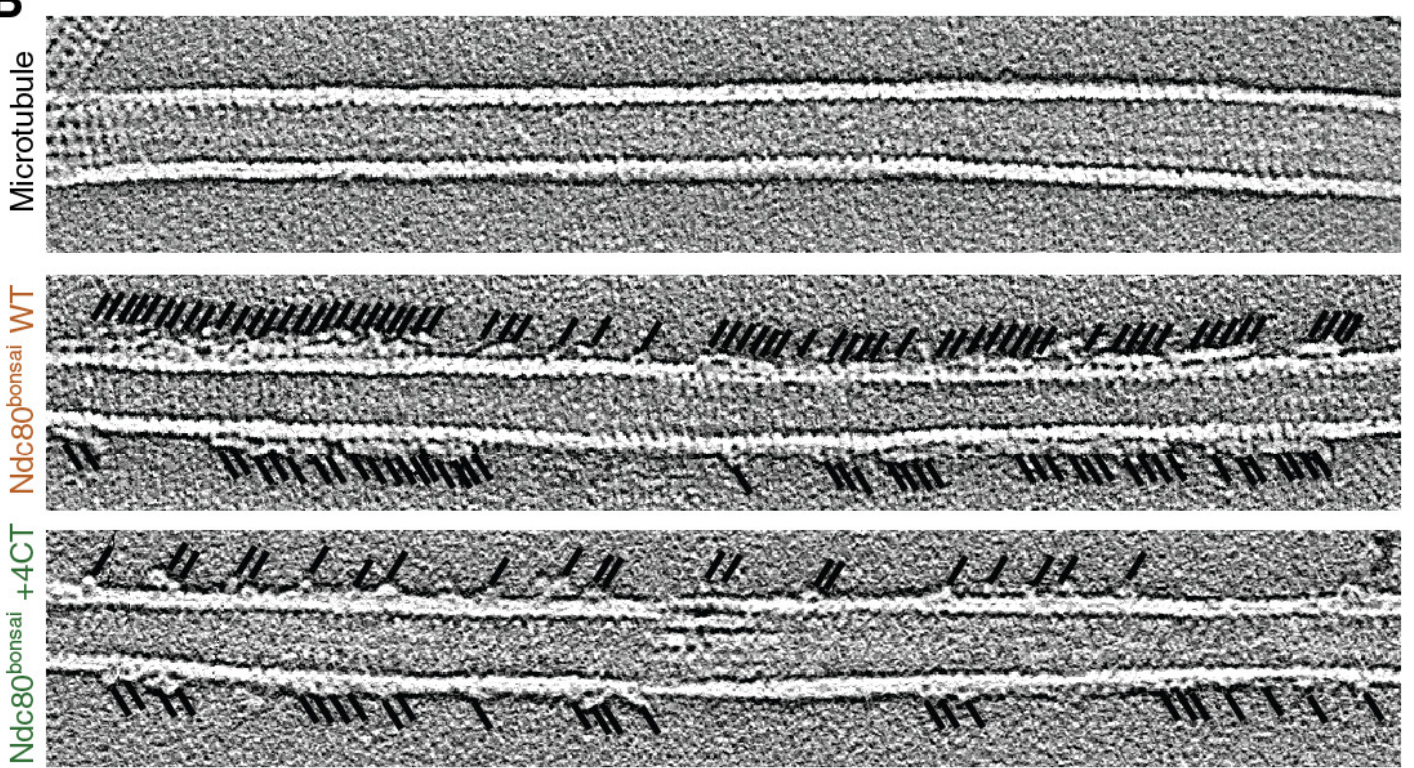

C
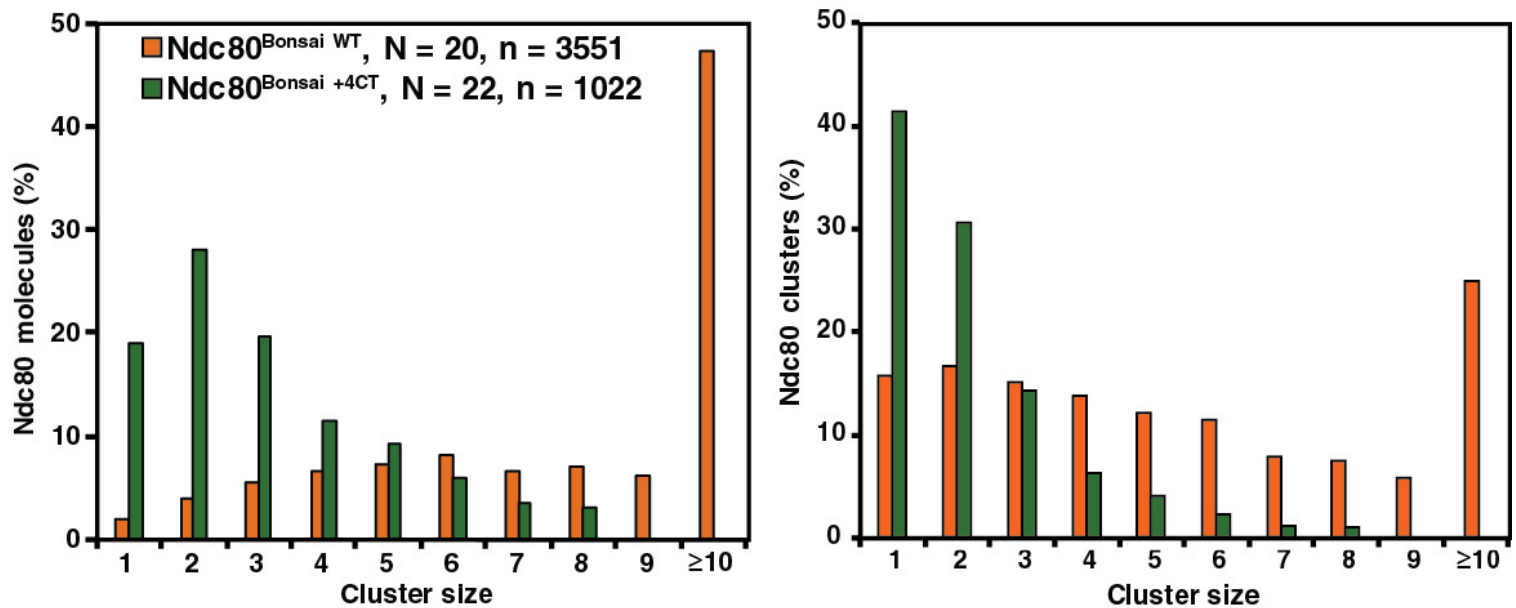

(A): Pawel Janczyk; (B,C): Katarzyna Skorupka and Pawel Janczyk 


\section{Figure 2.4 Ndc80+4CT mutant fails to recruit Ska3 to the kinetochore.}

(A) Schematic representation of the experiment in (A') and (B).

(A') Immunofluorescence staining shows reduced levels of Ska3 (left panel), but not SKAP (right panel), on the kinetochores of Ndc $80^{+4 \mathrm{CT}}$ stable cell lines. White bar $=5 \mu \mathrm{m}$

(B) Box and whisker plots representing the quantification of Ska (left panel) and SKAP (right panel) staining intensities on kinetochores (>100 kinetochores from at least 4 cells, $\mathrm{p}$ value was calculated from on the averages of Ska or SKAP signal intensities normalized to Ndc80 from each cell). a.u. = arbitrary units.

(C) Schematic representation of the experiment in (C') and (D).

(C') Immunofluorescence staining of $\mathrm{Ndc} 80^{\mathrm{WT}}$ and $\mathrm{Ndc} 80^{+4 \mathrm{CT}}$ stable cell lines treated with $3.3 \mu \mathrm{M}$ nocodazole and $2 \mu \mathrm{M}$ ZM447439 shows reduced levels of Ska3 (red) on kinetochores. White bar $=5 \mu \mathrm{m}$

(D) Box and whisker plot representing the quantification of the Ska staining intensities on kinetochores in cells treated with nocodazole and ZM447439 (>100 kinetochores from at least 4 cells, $p$ value was calculated from the averages of Ska or SKAP signal intensities normalized to Ndc80 from each cell). a.u. = arbitrary units.

(E) PP1 phosphatase activity on phospho-Ndc $80^{\text {Bonsai }} \mathrm{WT}$ is increased in the presence of Ska1/Ska2, in a concentration dependent manner. 
Figure 2.4

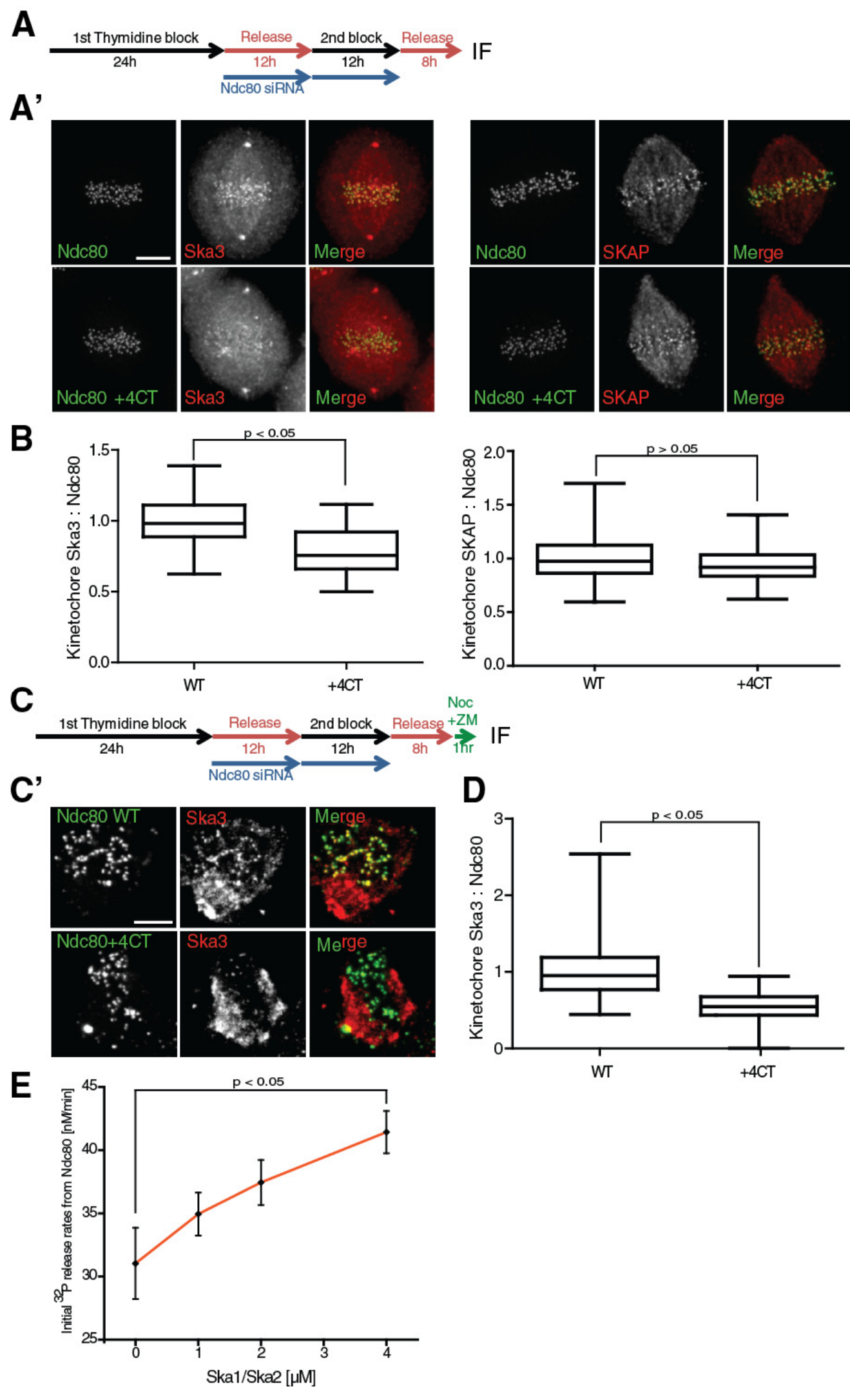

(A-B): Daniel Matson and Pawel Janczyk; (C-D): Pawel Janczyk and Thomas West (E): Pawel Janczyk 
Figure 2.5 Ndc80 interacts with Ska3 in both prometaphase and metaphase.

(A) Representative images of Ska3-Ndc80 Proximity Ligation Assay (red), with additional immunostaining of Borealin (cyan) and tubulin (green).

(B) Centromeres in prometaphase or metaphase cells, as identified by Borealin immunostaining, were scored based on the proximity to Ska3-Ndc80 PLA signal (>100 centromeres from at least four cells). Cells without one of the PLA probes were used as a control

(C) Centromeres in prometaphase or metaphase cells, as identified by Borealin immunostaining, scored based on the number of proximal Ska3-Ndc80 PLA signals ( $>100$ centromeres from at least four cells). Bars $=\mathrm{SD} . \mathrm{N} / \mathrm{S}-\mathrm{p}>0.05, *-\mathrm{p}<0.05, * * *-$ $\mathrm{p}<0.0001$ 


\section{Figure 2.5}

A

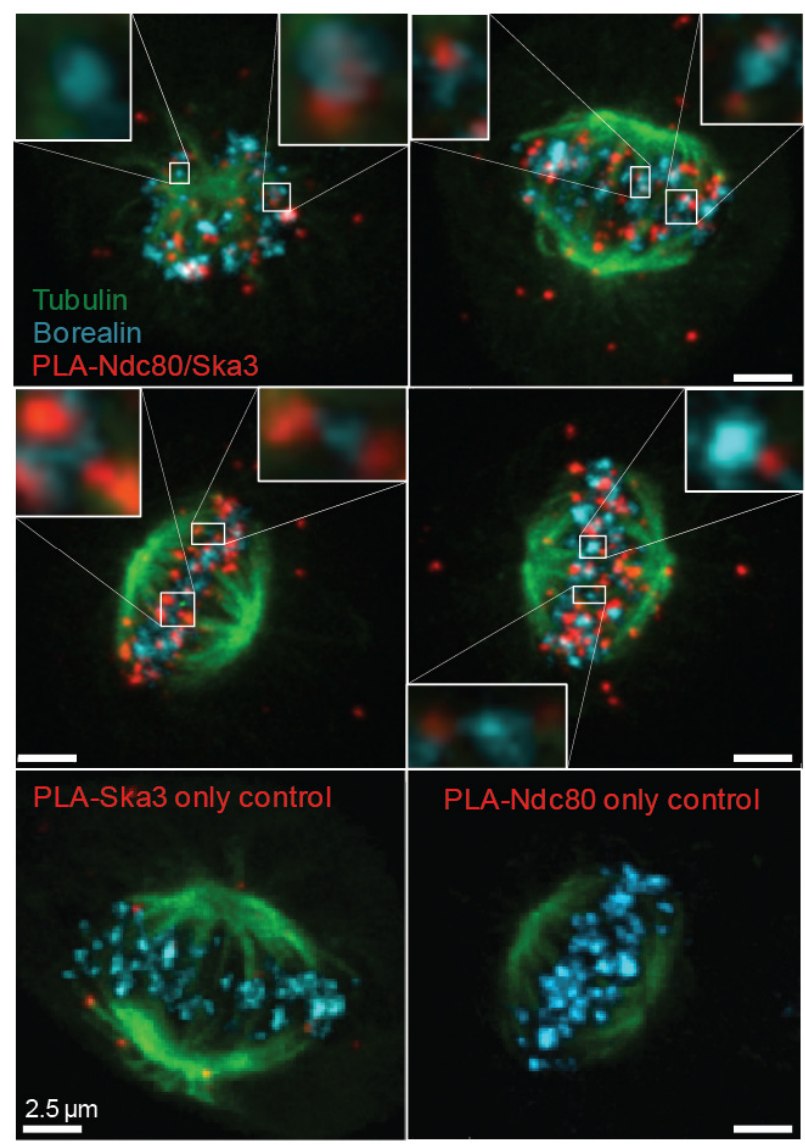

B

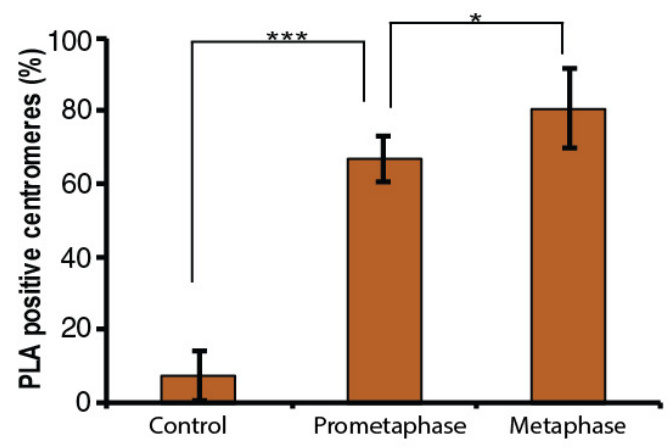

C

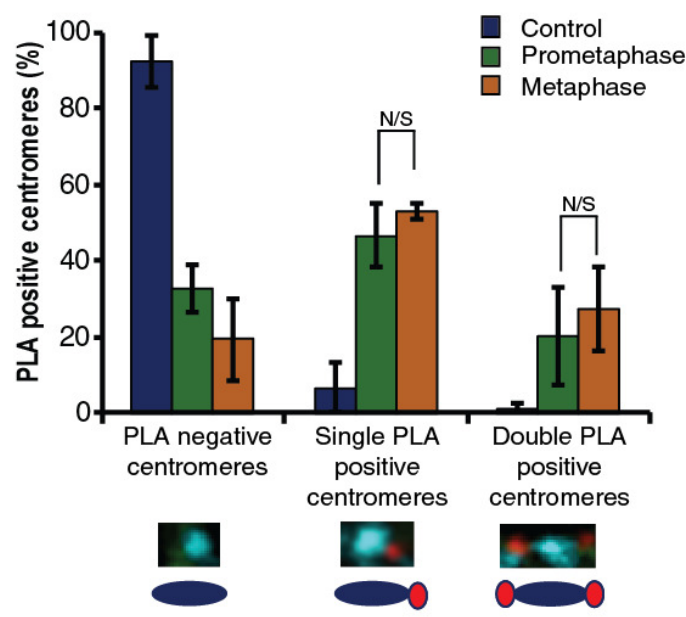

Cortney Kestner and Pawel Janczyk 
Figure 2.6 Ska forms V-shaped structures on microtubules in the presence of Ndc80

(A) Taxol-stabilized microtubules immobilized on the EM grids were sequentialy incubated with the $1 \mu \mathrm{M} \mathrm{Ndc} 80^{\text {Bonsai WT }}$ and $0.5 \mu \mathrm{M}$ human Ska, and subsequently stained with $2 \%$ uranyl formate. Beside previously described Ndc80 clusters a novel V-shaped structures can be observed on the microtubule lattice (yellow asterisk). Projection of 5 consecutive Z-sections of two representative microtubules is shown.

(B) Taxol-stabilized microtubules were incubated with the Ska complex (Xenopus laevis) and $\mathrm{Ndc} 80^{\text {Bonsai } W T}$, loaded on EM grids and stained with $2 \%$ uranyl formate. Similar Vshaped structures can be observed as shown in (A) (left, yellow asterisk) with $\mathrm{Ndc} 80$ clusters underneath (right, black lines indicating positions of Ndc80 molecules).

(C) Model for arrangement of Ndc80 (red) and Ska (green) molecules within the Vshaped structures. 
Figure 2.6

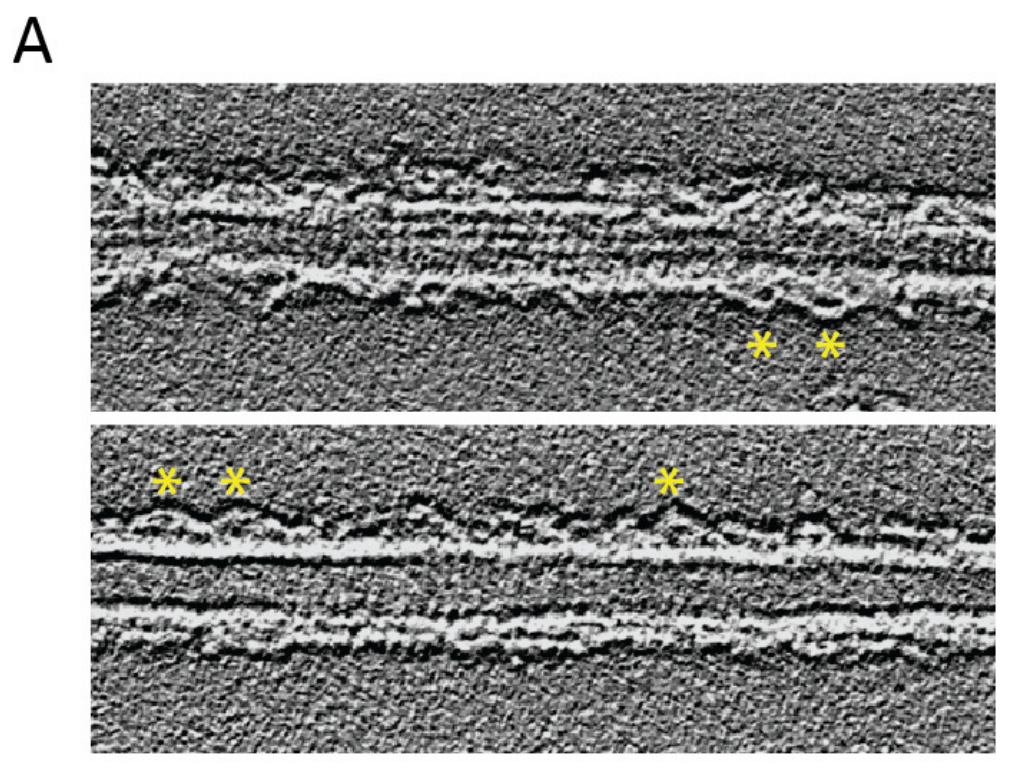

B
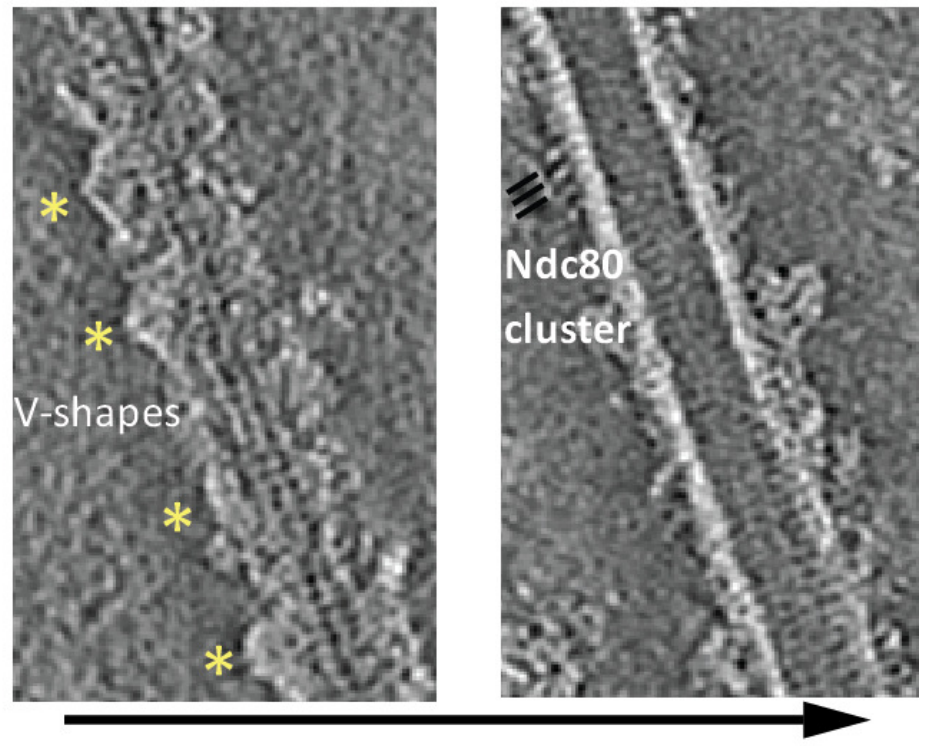

Z

C

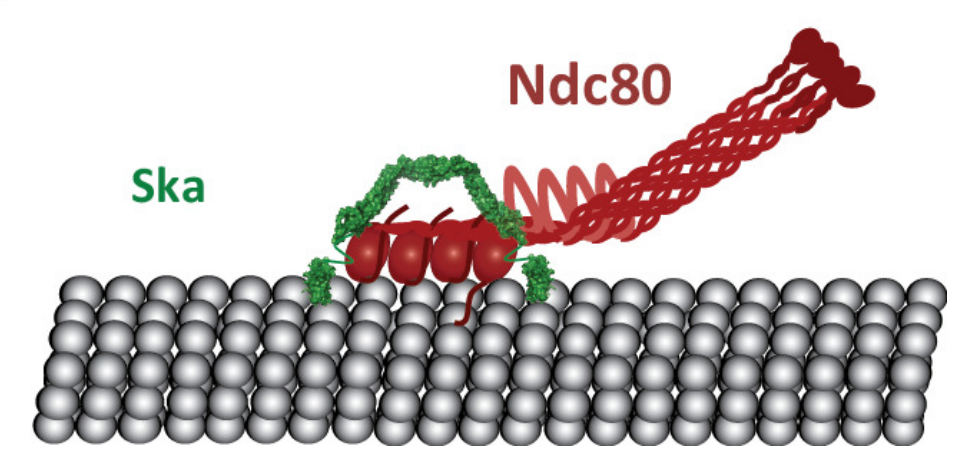

(A): Pawel Janczyk and Katarzyna Skorupka; (B) Gregory Alushin and Pawel Janczyk 


\section{Figure 2.7 Model of Ska recruitment to the kinetochores}

(A) Left - Ska binds to attached kinetochore-microtubules but the association with Ndc80 is limited as the clustering is inhibited by high Aurora B-dependent phosphorylation (yellow stars). Right - Ndc80 forms clusters on microtubules upon dephosphorylation allowing for the recruitment of Ska.

(B) Left - In presence of a microtubule depolymerizing drug, nocodazole, Ndc80 remains phosphorylated by Aurora B and does not form clusters and Ska cannot be recruited to kinetochores. Right - In presence of Aurora B inhibitor (ZM447439) Ndc80 remains unphosphorylated likely allowing for spontaneous clustering without microtubules, which in turn recruits Ska. 


\section{Figure 2.7}

A

Unaligned chromosome

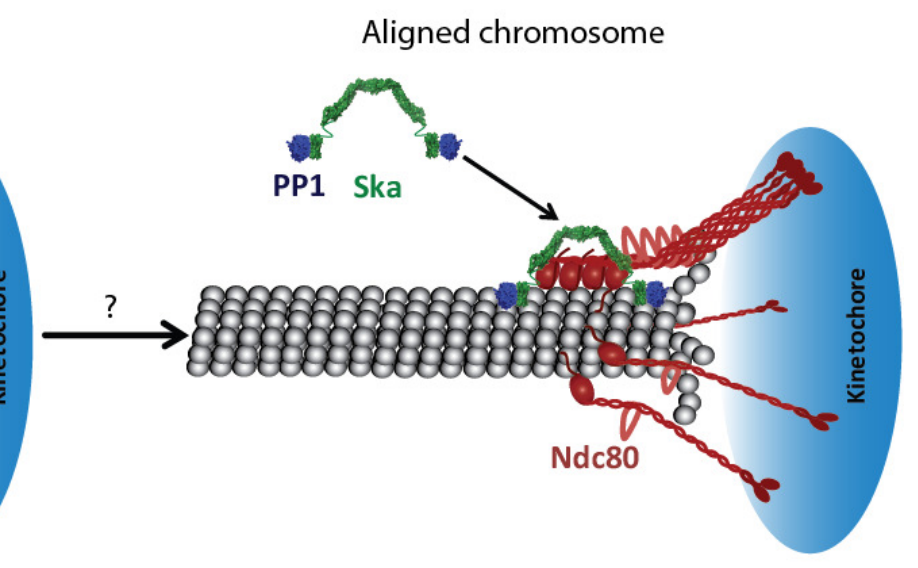

B
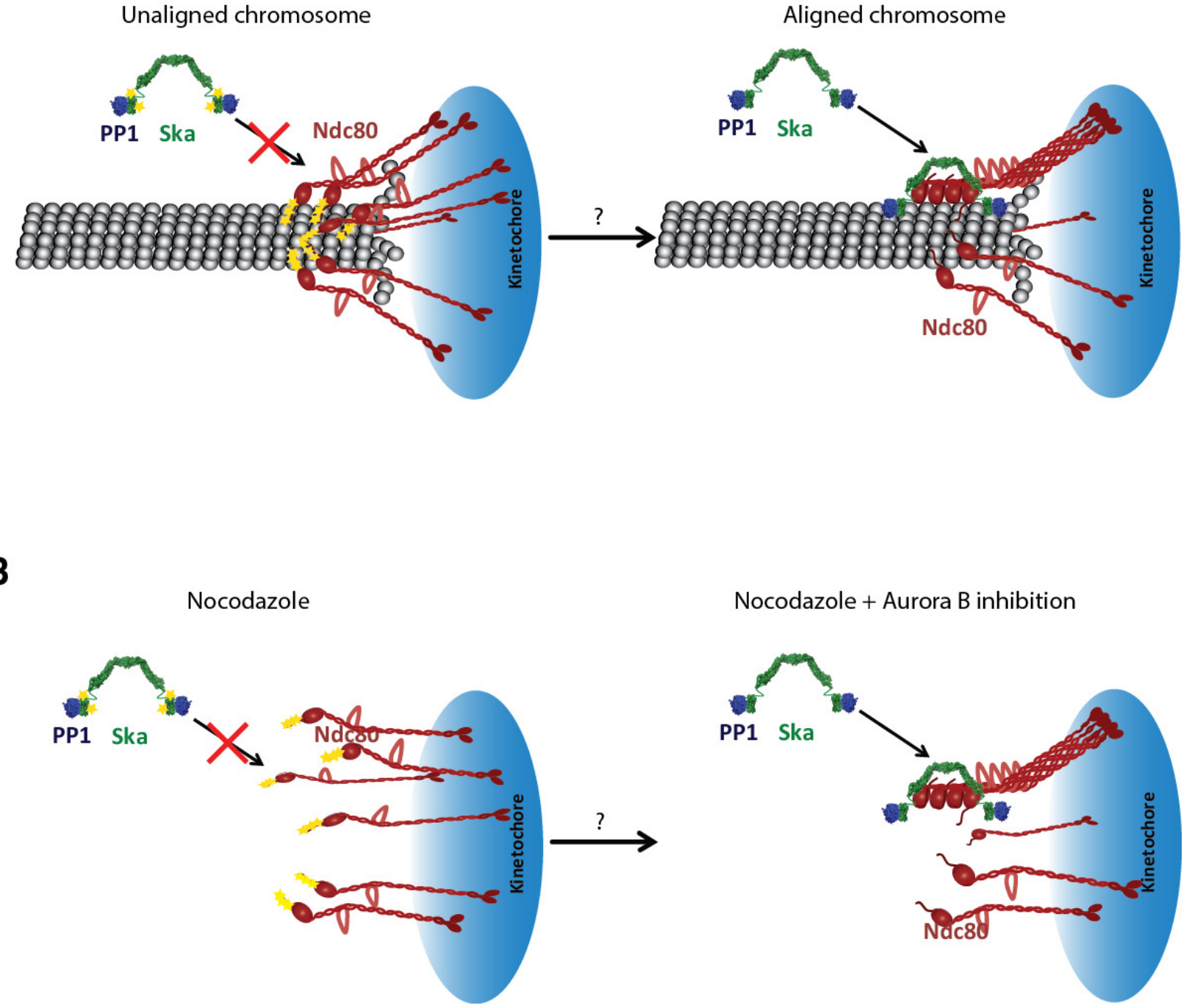


\section{Supplemental Figure 2.1. Characterization of the Ndc80 tail truncation mutants}

(A) Cartoon depicting the wild type Ndc80 tail and Ndc80 tail mutants used for knockdown and rescue experiments in HeLa cells. Positively charged residues are indicated by blue rectangles; negatively charged residues are indicated by red rectangles; Aurora B phosphorylation sites are indicated by green rectangles. Tail deletions are denoted by a solid black line; individual charge mutations to alanine are denoted by loss of blue rectangle. Gray ovals to the right of the 80 -amino acid tail depict the structured Calponin Homology Domain (CHD) and Coiled-Coil Domain (CCD) that comprise the remainder of the Ndc80 protein. Images not drawn to scale.

(B) Cells were incubated in ice-cold media for 15 minutes prior to fixation and immunofluorescence for tubulin (green), Ndc80 (red), and ACA (blue). Representative images of control cells from each mitotic phase are shown to demonstrate the scoring method.

(C) Ten kinetochores from five or more cells $(n>50)$ were scored for associated microtubules in two independent assays, and the mean percentage in early prometaphase, late prometaphase and metaphase was plotted. Error bars indicate standard deviation.

(D) Mitotic cells were scored for kinetochore alignment ( $\mathrm{n}>100$ for each condition). Metaphase cells were further subdivided to indicate cells with all chromosomes aligned (black), cells with few unaligned chromosomes (white) and cells with 3-5 unaligned chromosomes (white).

(E) Ten sister kinetochores in at least five cells $(n>50)$ were identified by ACA staining between $\mathrm{Ndc} 80$ signals and the distance between those sister kinetochores was measured in three independent experiments. The mean distance is plotted for early prometaphase and metaphase cells. Error bars indicate standard deviation. 
Supplemental Figure 2.1

A

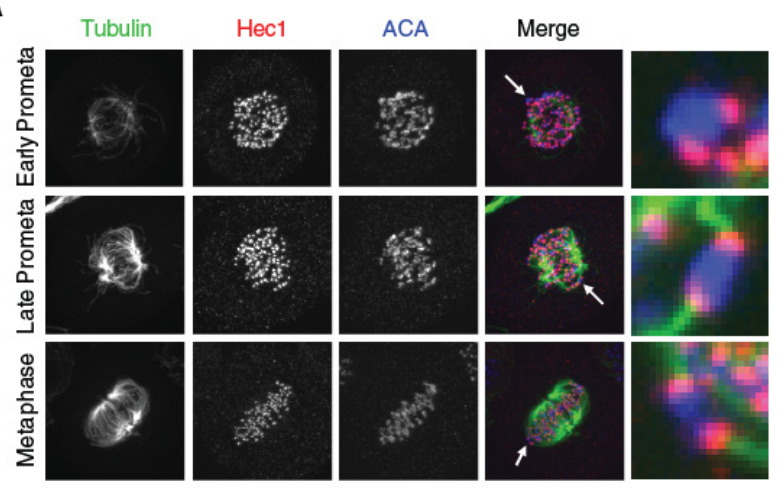

C

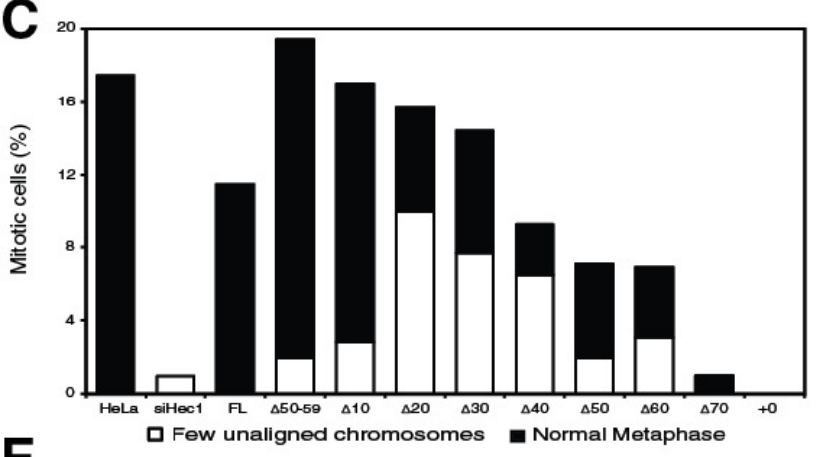

E

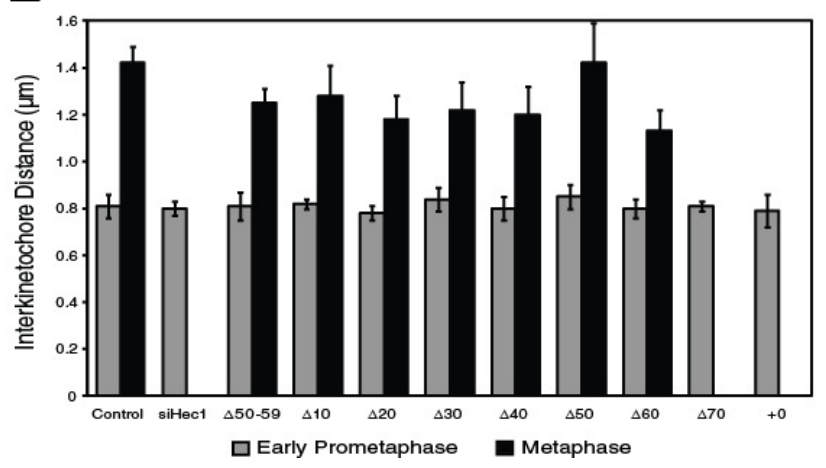

B

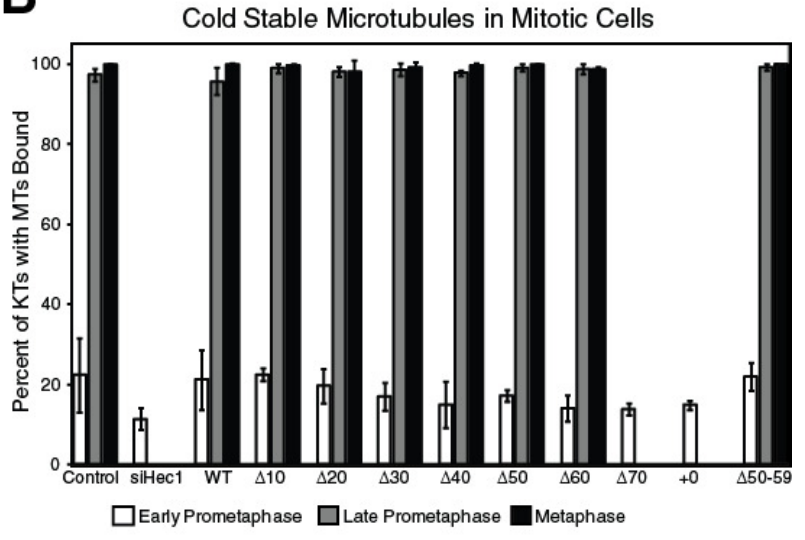

D

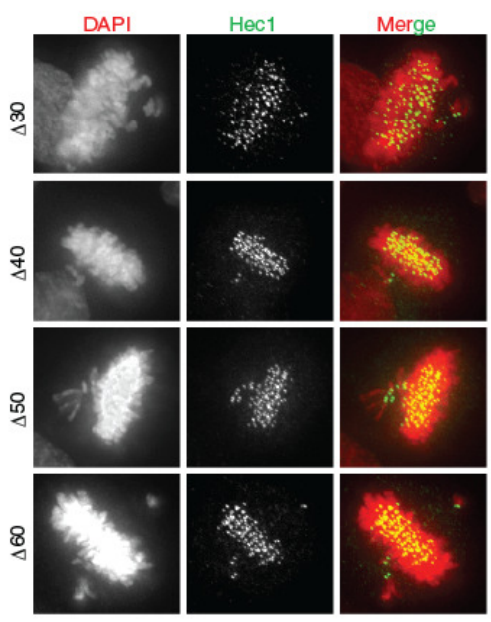


Supplemental Figure 2.2. Kinetochore assembly is not disrupted in the presence of Hec1 tail deletion mutants

Cells were immunostained for the indicated proteins. Representative prometaphase cells for each condition are shown. Images of ACA, Hec1, and Mad2 are maximum projections of the entire cell. Images of ACA, Spc25 and CENP-E are maximum projections of five slices at the center of each cell. 
Supplemental Figure 2.2

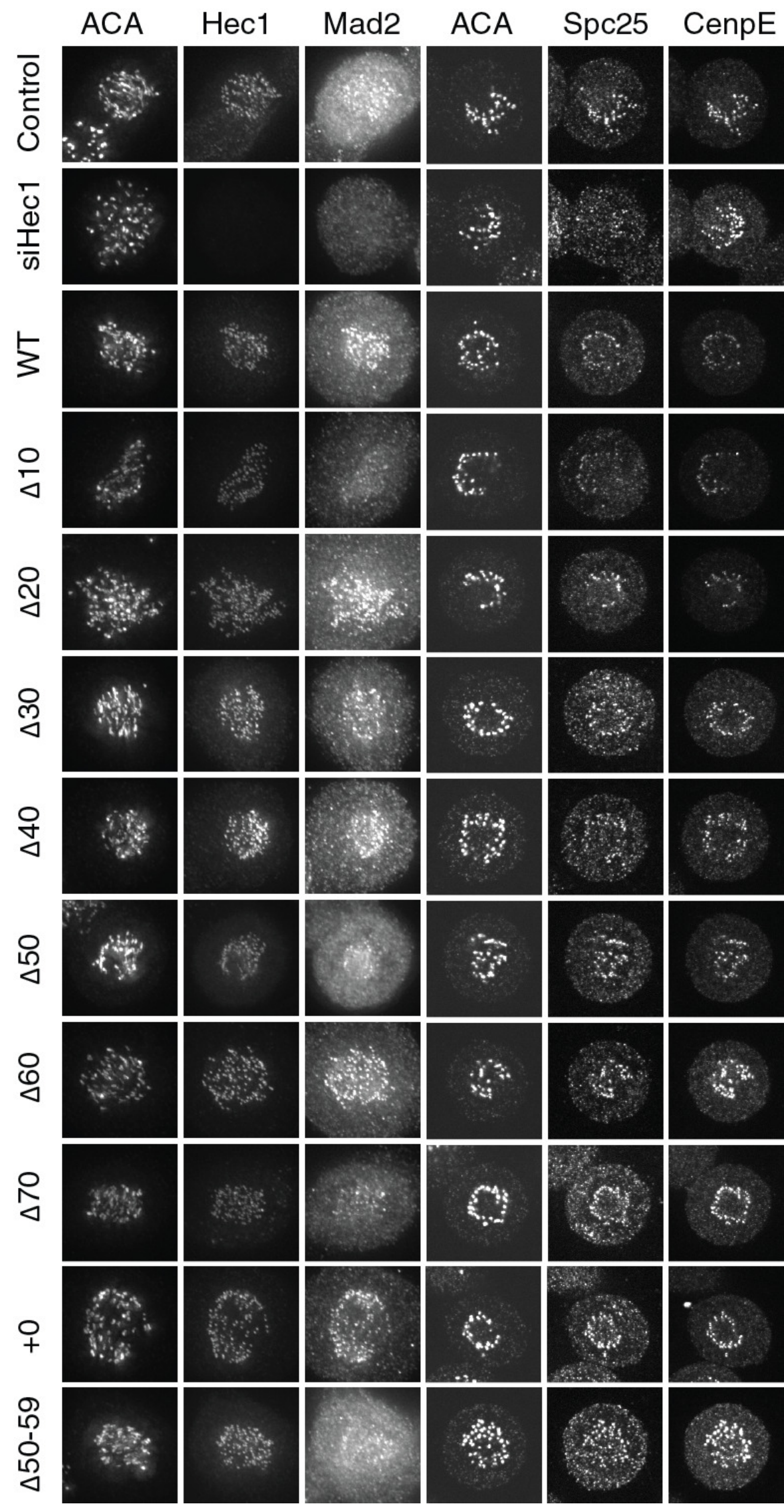

Stephanie Miller 


\title{
Chapter III
}

\section{The human Ska complex drives the metaphase-anaphase cell cycle transition by recruiting protein phosphatase 1 to}

\author{
kinetochores
}

This chapter is based on unpublished work and the following publication. Sivakumar, S., Janczyk, P.Ł., Qu, Q., Brautigam, C.A., Stukenberg, P.T., Yu, H., Gorbsky, G.J., "The human SKA complex drives the metaphase-anaphase cell cycle transition by recruiting protein phosphatase 1 to kinetochores" 2016. eLife 5 . doi:10.7554/eLife.12902 


\section{$\underline{\text { Abstract }}$}

The spindle- and kinetochore-associated (Ska) complex is essential for normal anaphase onset in mitosis. The C-terminal domain (CTD) of Ska1 binds microtubules and was proposed to facilitate kinetochore movement on depolymerizing spindle microtubules. Here, we show that Ska complex recruits protein phosphatase 1 (PP1) to kinetochores. This recruitment is facilitated by the Ska3 and the Ska1 CTD, which binds PP1 in vitro and in human HeLa cells. Ska1 lacking its CTD fused to a PP1-binding peptide or fused directly to PP1 rescues mitotic defects caused by Ska1 depletion. Ska1 fusion to catalytically inactive PP1 mutant does not rescue the Ska1 depletion phenotypes and shows dominant negative effects. Thus, the Ska complex, specifically the Ska1 CTD, recruits PP1 to kinetochores to oppose spindle checkpoint signaling kinases and promote anaphase onset. Microtubule binding by Ska, rather than acting in force production for chromosome movement, may instead serve to promote PP1 recruitment to kinetochores fully attached to spindle microtubules at metaphase.

\section{Introduction}

Anaphase onset and mitotic exit are driven by proteasome-mediated destruction of Securin and Cyclin B, which are targeted for ubiquitylation by the E3 ligase, the Anaphase-promoting complex/cyclosome (APC/C) [227]. Until metaphase, APC/C activity is restrained by the spindle checkpoint. Recent studies indicate that a key element in extinguishing checkpoint signaling when chromosomes align at metaphase is the displacement of the critical checkpoint signaling kinase, Mps1, from its substrate, Knl1, at kinetochores $[142,143,196]$. Then, to allow anaphase onset and mitotic exit, Mps1 phosphorylation events must be reversed by phosphatases. Previously, it was showed that depletion of the Ska complex leads to a strong metaphase arrest or delay [137,138]. Even 
when Mps1 was directly inhibited with a strong chemical inhibitor, cells depleted of the Ska complex exited mitosis more slowly [138]. These results suggested that the Ska complex played some role in opposition to, or downstream of, checkpoint signaling. Here, we provide evidence that the Ska complex recruits protein phosphatase 1 (PP1) to kinetochores, consistent with a role in opposing checkpoint signaling by kinetochoreassociated kinases.

Centromere- and kinetochore-associated kinases play key roles in regulating chromosome attachment to the spindle and cell cycle progression in mitosis. As chromosomes align to the metaphase plate, PP1 becomes concentrated at kinetochores stabilizing kinetochore-microtubule interactions, and promoting anaphase onset by opposing spindle checkpoint kinase signaling [188,198]. Recruitment of PP1 to the kinetochore then leads to substrate dephosphorylation, stabilization of kinetochoremicrotubule attachments, and anaphase onset [166,187,188,198,202].

The heterotrimeric spindle- and kinetochore-associated (Ska) protein complex consisting of Ska1-3 is required for timely anaphase onset [121,135,137]. Some laboratories have reported that Ska depletion strongly compromises chromosome alignment $[121,135,136]$. However, detailed video analyses show that depletion of Ska components, individually or in combination, causes delays in chromosome alignment followed by a robust metaphase arrest [137,138] Metaphase arrest is often then followed by cohesion fatigue and asynchronous chromatid separation without progression to anaphase [140,228]. Cells that have undergone cohesion fatigue remain arrested in mitosis with a terminal phenotype in which mixtures of separated chromatids and intact chromosomes are scattered across the spindle. 
The C-terminal domain (CTD) of Ska1 binds to microtubules and has thus been termed the microtubule-binding domain or MTBD [110,145]. The CTD of Ska3 was shown to contribute to the microtubule binding of the complex [144]. HeLa cells expressing Ska lacking CTD of either Ska1 or Ska3 showed phenotypes similar to Ska depletion [144]. Cells were delayed at metaphase, and cold stable kinetochore fibers were reportedly decreased $[110,145]$. Furthermore, the Ska complex was shown to track depolymerizing microtubule ends in vitro [110]. These results have led to a model in which Ska directly promotes microtubule stability and kinetochore movement on microtubules in conjunction with other kinetochore components, such as the Ndc80 complex $[110,145]$

In this study, we report that Ska recruits PP1 to kinetochores. Compromising this recruitment increases phosphorylation of Knl1 and increases recruitment of the checkpoint kinase Bub1. We find that Ska1 CTD is required for binding to PP1 in vivo and in vitro and Ska3 may contribute to that interaction, likely by multipartite contacts. The metaphase arrest or delay seen after Ska depletion is strongly rescued by expressing a chimeric Ska1 protein with its CTD replaced by PP1. Thus, a major function of the Ska complex is to recruit PP1 to the kinetochore. 


\section{$\underline{\text { Results }}$}

\section{The Ska complex is required for kinetochore accumulation of PP1}

Several mitotic kinases, including Mps1, Aurora B, Bub1 and Plk1 accrue to high levels at centromeres and kinetochores in early mitosis, during prophase and prometaphase [89]. An opposing phosphatase, PP1, also accumulates on kinetochores as microtubules attach, reaching maximal levels at metaphase [188,198,208,229]. We tested if the recruitment of PP1 to kinetochores was affected in Ska3-depleted cells. We found that the kinetochore levels of PP1 were diminished in Ska3-depleted cells at prometaphase and metaphase (Figure 3.1.A). In contrast, the pool of PP1 on the mitotic spindle appeared unaffected. Thus, the Ska complex is required to recruit PP1 to the kinetochore.

Since Ska depletion reduced PP1 recruitment to the kinetochore, we asked if overexpression of Ska would increase it. We had previously shown that expressing a fusion of the KMN component, Mis12, to Ska1 (Mis12-Ska1) increased total Ska recruitment to the kinetochore [138]. Cells expressing Mis12-Ska1 had higher levels of PP1 at kinetochores than control cells expressing either Mis12 or Ska1. The cells in this experiment were arrested at metaphase with the proteasome inhibitor, MG132 (Figure 3.1.B). Expression of Mis12-Ska1 also increased PP1 levels at kinetochores in cells treated with nocodazole (Figure 3.1.C), suggesting that targeting Ska complex to the kinetochore leads to accumulation of kinetochore PP1 in a microtubule-independent manner. Although expression of Mis12-Ska1 increased PP1 levels at the kinetochore, it was not sufficient to accelerate anaphase onset in normal cells or induce mitotic exit in nocodazole-treated cells [138]. 


\section{The Ska complex directly interacts with PP1 through Ska3 subunit.}

PP1 was shown to form complexes with over 200 regulatory subunits [230,231]. While Knl1 is currently believed to play a main role in PP1 recruitment to the kinetochores, cells expressing Knl1 truncation mutant lacking region responsible for interaction with PP1 do not exhibit strong mitotic arrest [166,198]. This suggests that another kinetochore component might be required to recruit a pool of PP1 to facilitate mitotic exit. The Ska complex was shown to be important for silencing spindle checkpoint as depletion of any of the Ska proteins results in prolonged mitotic arrest. Current model suggests that Ska complex is predominantly responsible for stability of kinetochore-microtubule attachments; however, Ska is dispensable for chromosomal alignment [137,138]. To determine if the Ska complex interacts with PP1 in vitro, we purified recombinant Ska3, Ska1/Ska2 and full Ska complex from E. coli and performed binding assays with purified recombinant PP $1 \alpha^{7-330}$. We observed a specific interaction between PP1 and Ska3 (Figure 3.2.A) with estimated apparent $K_{d}$ of $1.14 \mu \mathrm{M}$ (Figure 3.2.B).

The N-terminal end of Ska3 contains a highly conserved FxxR/KxR/K motif $\left({ }^{7}\right.$ FCGKLR $\left.{ }^{12}\right)$ that was previously implicated in binding with PP1 [232-235]. Although most of the PP1 binding partners interact through unstructured fragments/loops, this motif is often positioned within the helical structures (e.g. Bcl-xl, Bcl-w, AKAP5). Interestingly, in Ska3 this region is located at the N-termini of the triple helix bundle that forms in presence of Ska1 and Ska2. It is likely that Ska1 and Ska2 block the access to the $\mathrm{F} x \mathrm{xR} / \mathrm{KxR} / \mathrm{K}$ motif as mutations within that sequence $(\mathrm{F} 7 \mathrm{R} / \mathrm{L} 11 \mathrm{R})$ were shown to be important for proper assembly of the Ska core complex [144]. We therefore analyzed the potential Ska-PP1 interaction by size exclusion chromatography (SEC) with PP1 and the Ska complex, alone or premixed. Interestingly, we observed that the majority of PP1 co- 
migrated together with the Ska complex (Figure 3.2.C). This suggested that either FxxR/KxR/K motif is not essential for PP1 binding or the binding is not affected by the presence of Ska1/Ska2. SEC of Ska3 3A mutant $\left({ }^{7}\right.$ FCGKLR $^{12}$ mutated to ${ }^{7}$ ACGALA $^{12}$ ) resulted in similar shift in PP1 migration pattern indicating that this motif is not essential for Ska3-PP1 binding (data not shown).

\section{Interaction with PP1 is facilitated by multiple sites on Ska3.}

One of the hallmarks of the majority of the PP1 binding regions is their intrinsically disordered nature [230,231]. Ska3 contains over 300 amino acids long Cterminal region that was previously suggested to contribute to the microtubule binding and Ska recruitment to the kinetochores [121]. As this region is predicted to be predominantly unstructured, we investigated the possibility of its involvement in PP1 binding. We generated a series of GST-tagged Ska3 fragments and analyzed their ability to bind PP1. In vitro binding assay revealed that both 300 amino-acid long unstructured C-terminal region and full length Ska3 binds to PP1 (Figure 3.3.A). We also found that short Ska3 ${ }^{344-412}$ fragment at the C-terminal end was depleted from the solution by high concentrations of PP1. It suggested that this region might be important for PP1 binding. Surprisingly, when we analyzed migration pattern of PP1 on size exclusion chromatography in presence of $\mathrm{Ska}^{1-343}$ or Ska1/Ska2/Ska3 ${ }^{1-343}$, we observed similar shift as in presence of the full-length Ska3 (data not shown) or Ska1/Ska2/Ska3 (Figure 3.3.B, compare with Figure 3.2.C). It suggested that PP1 associates with multiple interfaces on Ska3. To investigate this possibility, we utilized fluorescence polarization binding assay with fluorescently labeled PP1. We observed that GST-Ska3 ${ }^{1-103}$ can interact with PP1 with the apparent affinity (Figure 3.3.C; $\mathrm{K}_{\mathrm{d}}=0.883 \pm 0.272 \mu \mathrm{M}$ ) slightly higher than the affinity of the full-length Ska3-PP1 interaction (Figure 3.2.A-B, $\left.\mathrm{K}_{\mathrm{d}}=1.142 \pm 0.423 \mu \mathrm{M}\right)$. It is important to note that the affinity of the GST-Ska3 ${ }^{1-103}$ was 
estimated in equilibrium, while full-length Ska3-PP1 affinity was measured by nonequilibrium type of assay. Surprisingly, we did not observe any significant reduction in the amounts of GST-Ska3 ${ }^{1-103}$ in our pull-down based PP1-binding assay (Figure 3.2.A).

\section{Ska1 CTD contributes to Ska-PP1 interaction.}

PP1 can be regulated by influencing its specificity, activity or by targeting to specific subcellular localization (or the combination of those) [231]. We therefore tested if Ska modulates the activity of PP1. We performed phosphatase activity assay on Ndc $80^{\text {Bonsai }}$ phosphorylated by Aurora. Interestingly, we observed a similar increase in the rates of ${ }^{32} \mathrm{P}$ release when either Ska1/Ska2 or Ska1/Ska2/Ska3 complex were present in the reaction mix ( $30 \%$ increase in velocity) (Figure 3.4.A). This indicated that Ska1/Ska2 stimulates PP1 phosphatase activity and Ska3 is dispensable for that stimulation. Previously we have shown that Ska1/Ska2 increases PP1 phosphatase activity (Chapter 2, Figure 2.4E), however, we were unable to detect any shift in migration pattern of PP1 in presence of Ska1/Ska2 as analyzed by SEC (data not shown). However, we observed a binding between PP1 and CTD of Ska1 by in vitro translation binding assay (data not shown, see [220] Figure 3A). It was previously suggested that the major function of this region is microtubule binding [110,145]. Using microscale thermophoresis (MST) we found that purified recombinant Ska1 CTD and PP1 proteins interacted with an apparent $\mathrm{K}_{\mathrm{d}}$ of $1.5 \mu \mathrm{M}$ (Figure 3.4.B). Thus, Ska1 CTD and PP1 directly interact with moderate affinity. Other parts of Ska or associated proteins may enhance the Ska1 CTD-PP1 interaction in vivo. We then assessed if that interaction is important for PP1 binding by the Ska complex. For the purpose of this experiment we used Ska complex that contained Ska3 $3^{1-343}$, as it was more stable than the complex containing full length protein. We observed a decrease in the amount of co-migrated PP1 
when complex lacking C-terminal domain of Ska1 was used (Figure 3.3.C). This indicates that Ska1 CTD contributes to, but is not exclusive for Ska-PP1 binding

\section{The Ska1 CTD is required for PP1 kinetochore targeting and Knl1 dephosphorylation}

Although we have shown that Ska3 contributes to Ska-PP1 interaction, depletion of Ska3 or its C-terminal unstructured tail results in mislocalization of the Ska complex from the kinetochores [121,144], making it difficult to study the importance of Ska3 in PP1 recruitment in vivo. Therefore, we focused on characterizing the role of Ska1 CTD in cells. Since Ska1 $\triangle \mathrm{CTD}$ was deficient in PP1 binding, we expected the cell line expressing this mutant to show reduced kinetochore localization of PP1. To eliminate potential PP1 binding to microtubules or microtubule-associated proteins, we treated the cells with high concentration $(3.3 \mathrm{mM})$ nocodazole PP1 localization to the kinetochore was decreased by $50 \%$ in cells expressing Ska1 $\triangle \mathrm{CTD}$, compared to cells expressing fulllength Ska1 (Figure 3.5.A), with or without depletion of Ska1. Thus, Ska1 $\Delta$ CTD could diminish PP1 kinetochore targeting in a dominant negative fashion, confirming a role for the Ska1 CTD in PP1 targeting to kinetochores.

The spindle checkpoint kinase Mps1 phosphorylates Knl1 on multiple MELT motifs, and the phosphorylated MELT recruits other checkpoint components, such as Bub1, to kinetochores $[164,168,236]$. We found that phosphorylation of one such MELT motif, pMELT (residue pT875), was indeed elevated in cells expressing Ska1 $\triangle \mathrm{CTD}$, as compared to cells expressing full-length Ska1 (Figure 3.5.B) [143]. Consistent with this finding, cells expressing Ska1 $\triangle \mathrm{CTD}$ showed increased levels of Bub1 protein at kinetochores, as compared to cells expressing full-length Ska1 (Figure 3.5.C). Differences in PP1, pMELT and Bub1 labeling were not simply due to differences in expression levels in the stable cell lines, since quantification showed that GFP signals in 
GFP-Ska1 and GFP-Ska1 $\Delta$ CTD cells were similar (data not shown, see [220]). These results indicate that PP1 recruited by the Ska1 CTD opposes Knl1 phosphorylation by Mps1 directly or indirectly.

\section{Ska-mediated kinetochore recruitment of PP1 promotes anaphase onset}

PP1 is recruited to the kinetochore to dephosphorylate kinetochore proteins to oppose spindle checkpoint signaling and promote anaphase onset. PP1 depletion was found to cause delays at metaphase $[166,188,198]$. Our experiments show that cells expressing Ska1 $\triangle$ CTD are deficient in PP1 recruitment to the kinetochore. We sought to determine whether the kinetochore recruitment of PP1 by Ska affected mitotic progression. Depletion of Ska proteins by RNAi causes long metaphase delays or arrest [138]. As expected, expression of RNAi-resistant Ska1 rescued the arrest caused by Ska1 depletion (Figure 3.6.A). Consistent with previous reports [110,145], expression of Ska1 $\triangle \mathrm{CTD}$ did not rescue the metaphase arrest/delay caused by Ska1 depletion (Figure 3.6.A,B).

To test if direct PP1 recruitment by Ska1 could promote anaphase onset, we created a chimeric Ska1 protein with its CTD replaced with PP1. Expression of the Ska1PP1 chimeric protein produced higher PP1 accumulation (1.5-fold increase) at kinetochores (data not shown), and resulted in complete rescue of the mitotic arrest and alignment delay phenotypes and substantially reduced the metaphase delay phenotype induced by Ska1 depletion (Figure 3.6.A). As a control, expression of PP1 alone, not fused to Ska1, failed to rescue. Thus, expression of a Ska1-PP1 chimeric protein, lacking the CTD and its associated microtubule-binding activity, significantly alleviates the mitotic phenotypes caused by Ska1 depletion. As shown previously [110] and confirmed here, expression of Ska1 $\triangle$ CTD caused metaphase delays even in cells without Ska1 depletion, indicating that it dominant negatively inhibits Ska function (Figure 3.6.B). 
This dominant-negative phenotype was not seen with the Ska1-PP1 chimeric protein in which the CTD was replaced by PP1 (Figure 3.6A, B). These results are consistent with a role for Ska1-recruited PP1 in mitotic progression.

To determine if the phosphatase activity of PP1 was essential for the rescue of Ska1-depleted cells expressing the Ska1-PP1 chimera, we generated an expression vector containing a fusion protein with a point mutation $(\mathrm{H} 248 \mathrm{~K})$ in PP1 that abolished its catalytic activity [237]. Expression of PP1 H248K (phosphatase dead PP1/pdPP1) without fusion to Ska1 was toxic to cells and induced cell death in interphase (data not shown). Expression of Ska1 $\Delta$ CTD-PP1 H248K (Ska1 $\Delta$ CTDpdPP1) allowed cells to enter mitosis, but failed to rescue the mitotic delay of Ska1-depleted cells (Figure 3.6.C, D, E). The chromosome alignment delays observed upon Ska1 depletion were also rescued by expression of Ska1-PP1 fusion protein but not by expression of Ska1 $\triangle \mathrm{CTDpdPP} 1$ (Figure 3.6.E). Indeed, mere expression of Ska1 $\triangle \mathrm{CTDpdPP1}$ without Ska1 depletion resulted in phenotypes remarkably similar to those caused by Ska1 depletion, indicating that this mutant acted in a dominant negative manner. These data indicate that a major function of the Ska complex is to recruit active PP1 to kinetochores.

\section{Supplemental results}

\section{B55 subunits of the PP2A complex interact with Ska3.}

Another protein phosphatase crucial for regulation of mitotic processes is PP2A. B55 and B56 protein families are known regulatory subunits of PP2A, important for its mitotic functions. The B55 family was previously implicated in the regulation of mitotic entry and exit [238-241]. However, despite detailed characterization of the role of B55 in mitotic entry, the mechanism of its functions in the mitotic exit is still poorly understood. Recent proteomic studies identified a connection between Ska complex and B55 $\beta$ and 
B55 $\delta[134,242]$. To test if B55 subunits interact with the Ska complex, we performed binding experiments with in vitro translated B55 subunits and recombinant Ska complex. GST-bound Ska complex was able to pull down B55 $\gamma$. We also observed a weak interaction between Ska and B55 $\beta$ (Supplemental Figure 3.1.A). Surprisingly, we did not detect any interaction with in vitro translated PP1 $\alpha$. It is likely due to low levels of translated PP1, post-translational modifications, competition by other components present in the reticulocyte lysates or insufficient concentration of the Ska complex on the resin. Similar results were observed when GST-tagged Ska3 was used (data not shown). All human B55 isoforms contain a putative PP1 binding motif (RVxF). As the Ska complex directly interacts with PP1, we hypothesized that PP1 binding should increase the amount of B55 subunits pelleted by Ska-coupled resin. To test that hypothesis, we performed pull down experiments with recombinant GST-tagged Ska complex and B55 subunits in vitro translated together with PP1 $1 \alpha$. To increase the sensitivity of the assay we used low concentrations of the Ska complex on the resin (non-detectable by Coomassie staining of the elutions, compare with Supplemental Figure 3.1.A). Interestingly, we observed an increase in the amount of B55 subunits eluted from Ska resin when PP1 was present, in particular B55 $\alpha$ and B55 $\beta$ but not B55 $\gamma$ (Supplemental Figure 3.1.B). This might be due to PP1 direct interactions with Ska and B55. However, we did not observe any increase in the amount of PP1 in Ska pull-downs, suggesting that the stimulation of Ska-B55 interaction is caused by PP1 indirectly, e.g. by dephosphorylation of either Ska or B55 subunits. 


\section{$\underline{\text { Discussion }}$}

Before the alignment of chromosomes at the metaphase plate, the spindle checkpoint inhibits anaphase onset through signals generated by protein interactions at unattached kinetochores. This signaling requires the KMN complex, composed of the Knl1 protein, the Mis12 protein complex, and the Ndc80 protein complex. The central checkpoint kinase, Mps1, phosphorylates several MELT motifs on Knl1. The Bub1Bub3 complex binds to phosphorylated MELT motifs and further recruits its binding partner, the BubR1-Bub3 complex $[164,165,168,236]$. Mps1 also phosphorylates Bub1 to promote recruitment of the Mad1-Mad2 protein complex $[164,236]$. In the absence of microtubules, Mps1 associates with kinetochores via the Calponin homology domains (CHD) of the Ndc80 complex [106,142,143,162,243]. Photobleaching studies show that Mps 1 binding to kinetochores is highly dynamic, with a turnover time of $\sim 13 \mathrm{~s}$ [197]. The binding of microtubules to the Ndc80 complex displaces Mps1, and in budding yeast, this results in a rearrangement of kinetochore substructure such that Mps1 loses access to Knl1 [196]. In metazoans, microtubule binding to Ndc80 displaces Mps1 from kinetochores [142,143]. Thus, displacement of Mps1 from the Ndc80 complex by microtubule binding appears to be a central element of turning off kinase signaling. In metazoans, dynein "stripping" of checkpoint proteins, including Mad1, Mad2 and BubR1, plays an additional role in down regulating the checkpoint signal.

The most prominent phenotype of Ska complex depletion is arrest or long delay at metaphase, and these phenotypes require intact spindle checkpoint signaling. Abrogating checkpoint signaling with a chemical inhibitor of Mps1 induces mitotic exit in cells arrested in mitosis with high concentrations of microtubule poisons, but this exit is slower in cells depleted of Ska [138]. 
This result is consistent with the idea that Ska functions in opposition to and downstream of checkpoint signaling. Here, we show that the Ska complex is required for full kinetochore recruitment of PP1, a likely candidate for reversing checkpoint kinase phosphorylations. The fact that Ska depletion cannot completely block mitotic exit when Mps1 inhibitors are added likely reflects the fact that other pools of PP1 or other phosphatases, particularly PP2A, may also play a role in mitotic exit $[19,188,240]$. However, the strong metaphase arrest phenotype seen after Ska depletion attests to the importance of Ska-associated PP1 in regulating the metaphase-anaphase transition in normal mitosis.

We found that the Ska complex is essential for binding PP1 and recruiting it to the kinetochore. Ska3 shows multipartite association with PP1 in vitro, however it is also required for kinetochore targeting of the Ska complex [121]. As we were unable to find a mutant that separates those functions, we focused on characterization of the interaction between PP1 and Ska1, specifically the Ska1 CTD, previously shown to bind microtubules. Since the C-terminal domain is involved in binding to both PP1 and microtubules, we propose to name this domain simply as the CTD, instead of the MTBD. Consistent with a role in opposing Mps1 checkpoint signaling, we find that in cells where PP1 binding to Ska1 is compromised, there is a $30 \%$ increase in phosphorylation of a MELT motif on Knl1 and a 20\% increase in recruitment of Bub1 (Figure 3.5.B, C). A similar (30-40\%) increase in MELT phosphorylation and Bub1 levels also occurs upon mutation of Knl1 to inhibits its binding to PP1 [188]. We suggest that the pools of PP1 at the kinetochore are distinct and may play cooperative and specific roles at different stages of mitosis. It remains possible that there are unidentified targets whose dephosphorylation is more reliant on PP1 bound to Ska. The increase in Bub1 levels that we observe is also consistent with previous finding that Bub1 levels on kinetochores were increased in cells 
depleted of Ska3 [137]. Although Ska does localize at kinetochores not attached to microtubules, it accumulates to its highest levels on kinetochores of cells at metaphase, the moment in time when quickly reversing checkpoint-dependent phosphorylation would be most useful in initiating anaphase.

The CTD of Ska1 has been well characterized structurally. It has the characteristics of a winged-helix domain, a fold previously implicated in DNA binding and in mediating protein-protein interactions $[110,145]$. It was shown that the Ska1 CTD binds microtubules through multiple sites and can bind both straight and curved microtubule protofilaments $[110,145]$. The same Ska1 CTD is also required for PP1 binding.

Because the Ska1 CTD binds to both microtubules and PP1, its functions in promoting chromosome alignment and anaphase onset might be a consequence of either or both activities. In previous work, it was shown that mutation of three conserved arginine residues in the Ska1 CTD (R155A/R236A/R245A) compromised binding to microtubules in vitro and produced only a partial rescue of the metaphase delay caused by Ska1 depletion $[110,145]$. We found that this mutant of Ska1 R3A was still able to coprecipitate PP1 in extracts from cells where both were expressed as transgenes (data not shown). On the other hand, complete replacement of the CTD by a PP1-binding motif or by PP1 itself resulted in nearly complete rescue of all Ska1 depletion phenotypes, suggesting that microtubule binding by the Ska complex can be made dispensable. It was previously reported that Ska depletion does not impair chromatid separation in cells induced to enter anaphase by application of a chemical Cdk1 inhibitor [138]. Based on that work and the data presented here, we propose that the microtubule-binding properties of Ska1 do not play a strong mechanical coupling function for kinetochore movement on microtubules. We were unable to find a Ska1 point mutant that retained microtubule 
binding but was deficient in PP1 binding to further test this idea. Given the clear molecular evidence for Ska binding to microtubules and to microtubule protofilaments, we favor the idea that microtubule binding to Ska serves a regulatory role. We propose that Ska1 CTD binds near the ends of kinetochore microtubules, where separated protofilaments are enriched. This binding in concert with Ska complex interactions with other kinetochore components, may control the local concentration, dynamics, or substrate specificity of the Ska-PP1 complex at kinetochores (Figure 3.7).

PP1 was first identified as an important regulator that counters spindle checkpoint signaling in budding yeast and fission yeast [204,205]. The N-terminus of all Knl1 homologs from yeast to mammals contain conserved PP1-binding motifs [198]. In budding and fission yeast, expression of Knl1 mutants unable to bind PP1 impaired the ability of the cells to overcome checkpoint signaling and strongly compromised cell growth [187,202]. In C. elegans embryos, RNAi-mediated depletion of wild type Knl1 and its replacement with a PP1-binding mutant led to slow chromosome congression, delays at metaphase, and partial embryonic lethality [244]. The role of Knl1 binding of PP1 was studied in mammalian cells treated with nocodazole to disrupt microtubules and induce a strong spindle checkpoint arrest. Under these conditions, cells in which Knl1 was replaced with a PP1-binding mutant showed slower mitotic exit in comparison to controls when spindle checkpoint signaling was experimentally extinguished with a chemical inhibitor of Mps1 [162]. Together these studies have led to a model in which PP1 binding by Knl1 is a key factor in opposing checkpoint signaling for promoting the onset of anaphase and mitotic exit. However, one important result argues that this model does not fully explain the regulation of the metaphase-anaphase transition in normal mammalian cell mitosis. In mammalian cells with intact spindles, not treated with microtubule drugs, replacement of wild type Knl1 with a mutant Knl1 unable to bind PP1 
results in only a modest, 10-min delay at metaphase [166]. In contrast, loss of PP1 recruitment by the Ska complex during normal mitosis causes a lengthy delay or complete arrest at metaphase. Importantly, Ska homologs have not been identified in budding or fission yeast, consistent with the importance of PP1 recruitment by Knl1 in those organisms. In C. elegans, a two-protein Ska complex is present [110]. However, RNAi and mutant studies on Ska homologs in C. elegans embryos have not revealed an essential role in chromosome segregation (Arshad Desai, personal communication). Interestingly, an elegant approach for manipulating protein interactions within kinetochores in budding yeast at nanometer resolution indicated that recruitment of the yeast PP1 homolog to outer kinetochores was important for reversing Mps1 phosphorylations of Knl1 [196]. In mammalian cells with intact mitotic spindles, our study suggests that Ska, an outer kinetochore protein complex, is a critical recruiter of PP1 in opposing spindle checkpoint kinase signaling at kinetochores.

Our data indicate that binding of Ska and binding of Knl1 to PP1 are independent, suggesting that multiple pools of kinetochore-associated PP1 may cooperatively counter kinase activities at kinetochores. Their functions may be additive, recruiting PP1 to the threshold level required for anaphase onset. Interestingly, similar to the Ska1 CTD, the $\mathrm{N}$-terminal region of Knl1 adjacent to its PP1-binding motif also binds microtubules in vitro [42]. It is conceivable that the microtubule-binding domains of Knl1 and Ska1 may each regulate their associated PP1 pools, allowing them to be sensitive to the attachment status of the kinetochore. In addition, several other PP1-interacting proteins, including CENP-E, SDS22 and Repo-man, have been identified as playing roles in mitosis [245247]. However, these proteins, when expressed at endogenous levels, do not normally accumulate at kinetochores of metaphase chromosomes [245,248,249]. 
During other stages of mitosis, prometaphase and anaphase, they may function in regulating PP1 activities on kinetochores, chromosome arms, and in the cytoplasm [248251]. In the future, it will be important to determine which specific protein phosphorylations are targeted by Ska-PP1 or by other PP1-binding proteins during mitosis. Finally, it is clear that PP2A, and possibly other phosphatases also play vital roles in regulating phosphorylation to control chromosome movement and cell cycle progression in mitosis $[188,210,212,229,240,252,253]$.

In summary, here we make the surprising discovery that a chimeric Ska1-PP1 fusion lacking the microtubule-binding domain of Ska1 rescues nearly all the mitotic phenotypes observed upon Ska depletion, including delays in chromosome alignment and metaphase arrest. This rescue is fully dependent on the phosphatase activity of the chimera. Moreover, when expressed on its own, the phosphatase-dead Ska1-PP1 chimera has dominant phenotypes that closely mimic those of Ska depletion. Thus, rather than serving a mechanical coupling function between kinetochores and microtubules, the microtubule-binding properties of the Ska complex may primarily aid in coordinating $\mathrm{PP} 1$ recruitment to, or activity at, kinetochores. Our data suggest that PP1 recruitment is a critical function of the Ska complex for opposing mitotic kinases that destabilize kinetochore-microtubule attachment and that signal the spindle checkpoint. Thus, the Ska complex may integrate chromosome alignment at metaphase with full recruitment of PP1, thus opposing spindle checkpoint kinases signaling and promoting the metaphaseanaphase transition. 


\section{Material and Methods}

\section{Plasmids, protein purification and antibodies}

7xHis-Ska 1/Ska2, untagged Ska1/Ska2 and GST-Ska3 constructs were a gracious gift from Iain Cheeseman. GST tagged Ska3 fragments were created by introduction of a single stop-codon at appropriate positions in GST-Ska3 containing plasmid. GST-tagged Ska fragments were purified as follows. BL21 (DE3) cell lysate with Ska fragments were lysed (Emulsiflex C3 high pressure homogenizer, Avestin) in Binding Buffer (PBS + $0.05 \%$ Tween-20) and, after pre-clearing by 1 hour spin at 45,000 rpm (Beckman type 45 Ti rotor), incubated with Glutathione 4 Fast Flow Sepharose (GE Healthcare Life Sciences) for 1 hour, followed by $300 \mathrm{~mL}$ wash with Binding Buffer. Proteins were eluted with PBS $+0.05 \%$ Tween-20 $+15 \mathrm{mM}$ L-Glutathione Reduced and, after concentration on Vivaspin $43 \mathrm{~K}$ spin-columns, subjected to size exclusion chromatography on Superdex 75 10/300 GL column equilibrated with GF Buffer (50mM Tris, $\mathrm{pH}$ 7.5, $150 \mathrm{mM} \mathrm{KCl,} 1 \mathrm{mM}$ DTT). Purification of tag-less Ska3 and Ska Complex was done according to previously established protocol [145]. Plasmid containing hPP1 $\alpha$ 7-330 was a gracious gift from Wolfgang Peti. Purification of PP1 $\alpha$ was done as described in Materials and Methods section of Chapter 2. For in-vitro translation B55 subunits were obtained from human Orfeome 5.1 and cloned to pCSF107mTGATEWAY-3'-FLAG vector (Addgene \#67619) by Gateway cloning (ThermoFisher Scientific).

The Mis12-GFP, Ska1-GFP, Mis12Ska1-GFP plasmid construction has been described previously [138]. To construct fragments of Ska1, cDNA encoding indicated regions of Ska1 were PCR amplified from full length siRNA-resistant Ska1 and inserted 
in pCS2-GFP or GFP-N1 plasmid. pCS2-Myc-PP1 was made by inserting PP1 g into pCS2-Myc vector.

To construct fragments of Ska1, cDNA encoding indicated regions of Ska1 were PCR amplified from full length siRNA-resistant Ska1 and inserted in pCS2-GFP or GFPN1 plasmid. pCS2-Myc-PP1 was made by inserting PP1 $\gamma$ into pCS2-Myc vector. All plasmids were verified by DNA sequencing.

\section{In vitro binding and size exclusion co-migration assays.}

For protein pull-down experiments $1 \mathrm{~mL}$ of $1 \mu \mathrm{M}$ PP1 $\alpha$ 7-330 covalently linked with $20 \mu \mathrm{L}$ of CNBr-Activated Sepharose 4 Fast Flow according to manufacturer's protocol. Concentration of the protein remaining in the supernatant after reaction was measured by Bio-Rad Protein Assay to estimate PP1 concentration on the beads. PP1 bound beads were mixed with unbound resin in various ratios to obtain appropriate effective concentrations of PP1 for pull-down assays. After three $1 \mathrm{~mL}$ washes with GF Buffer resin was incubated for 1 hour at $4^{\circ} \mathrm{C}$ with $200 \mathrm{nM} \mathrm{Ska3}$ or GST-Ska3 fragments. For experiment depicted in Figure 3.2.B supernatants were collected and analyzed by SDS-PAGE and Western Blotting. For experiment depicted in Figure 3.1.A resin was washed two times with GF Buffer and pulled-down proteins were eluted with GF Buffer supplemented with $15 \mathrm{mM}$ L-Glutathione Reduced and subsequently analyzed by SDSPAGE and Western Blotting.

All size exclusion co-migration experiments were performed in $50 \mathrm{mM}$ HEPES, pH 7.5, $150 \mathrm{mM} \mathrm{KCl,} 1 \mathrm{mM}$ DTT on Superose 12 PC 3.2/30 size exclusion column (GE Healthcare) connected to AKTAmicro system (GE Healthcare). For experiments depicted in Figure 3.2.C and Figure 3.3.B Ska proteins were pre-incubated for 1 hour at ice with PP1 $\alpha$ 7-330, in 4:1 molar ratio. For experiment presented in Figure 3.4.C Ska proteins 
and PP1 were mixed in 2:1 molar ratio. $50 \mu \mathrm{L}$ fractions were collected and analyzed by SDS-PAGE and Western Blotting.

Antibody raised against hSka3 was used for detection (1:5000; generous gift from Gary Gorbsky [137]). GST-Ska3 fragments were detected by anti-GST antibody (1:1000; Pierce) and PP1 by chicken IgY antibody raised against PP1 $\gamma$ (1:5000; gracious gift from David Brautigan).

\section{Microscale thermophoresis (MST)}

To perform MST analysis, the GST-Ska1 CTD and His-PP1 were purified from bacteria. GST-Ska1 CTD was purified as described above and His-PP1 $\gamma$ was purified as described previously [231]. Briefly pET28a-His-PP1 $\gamma$ and a chaperone pGro7 (expressing GroESGroEL) was transformed into BL21 cells and protein expression was induced overnight at $10^{\circ} \mathrm{C}$ using L-Arabinose and IPTG. The bacterial pellets were lysed in lysis buffer (50 $\mathrm{mM}$ Tris $\mathrm{pH} 8.0,5 \mathrm{mM}$ imidazole, $700 \mathrm{mM} \mathrm{NaCl}, 1 \mathrm{mM} \mathrm{MnCl}_{2}, 0.1 \%$ Triton $\left.\mathrm{X}-100\right)$ and sonicated. The lysate was cleared by centrifugation at $\sim 25,000 \mathrm{~g}$ for $1 \mathrm{hr}$. The supernatant was incubated over $\mathrm{Ni}^{2+}$-NTA resin (Qiagen, Valencia, CA) to allow His-PP1 $\gamma$ binding. For MST analysis, the His tag was cleaved using Thrombin. The GST tag was cleaved from Ska1 CTD using 3C protease. The untagged proteins were eluted, further purified using Superdex size exclusion columns and concentrated using Amicon ultra centrifugal filters (GE Healthcare). Finally both Ska1-CTD and PP1 proteins were exchanged into MST buffer (25 mM HEPES, $50 \mathrm{mM} \mathrm{NaCl}, 1 \mathrm{mM}$ TCEP).

For MST, PP1 was covalently coupled to a fluorophore by incubating $200 \mu 1$ of PP1 at a concentration of $40 \mu \mathrm{M}$ with $1 \mu \mathrm{l}$ of $40 \mathrm{mM}$ Alexa-Fluor 488-Nhydroxysuccinamide ester (Molecular Probes/Life Technologies, Grand Island, NY) dissolved in $100 \%$ dimethyl sulfoxide) for $30 \mathrm{~min}$ in the dark at room temperature. The 
labeled protein was separated from free dye by applying the mixture to a G25 column that had been equilibrated with $9 \mathrm{ml}$ of protein storage buffer. Serial dilutions (1:1) of Ska1 CTD were made in 15 successive $10 \mu 1$ reactions, resulting in 16 samples, with the highest concentration of Ska1 CTD being $40 \mu \mathrm{M}$. Each of the reactions was mixed with $10 \mu 1800 \mathrm{nM}$ labeled PP1. Thus, the final concentration of the labeled protein was 400 $\mathrm{nM}$ in all samples, and the final highest concentration of Ska1 CTD was $20 \mu \mathrm{M}$; all reaction mixtures were supplemented with Tween-20 (NanoTemper, LLC, Munich, Germany) to a final concentration of $0.05 \%(\mathrm{v} / \mathrm{v})$. After incubation at room temperature for approximately $30 \mathrm{~min}$., all the reactions were loaded into standard treated capillary tubes, and the final measurements were taken in a Monolith NT.115 instrument (Nanotemper LLC, Munich, Germany). The instrument's LED (illumination) power was set to $25 \%$ and the MST laser power was set to $40 \%$. Measurements were performed at ambient temperature, ca. $23^{\circ} \mathrm{C}$. The times of data acquisition were $5 \mathrm{~s}$ before the activation of the MST laser, $30 \mathrm{~s}$ with the laser on, and $5 \mathrm{~s}$ after extinguishing the laser. Data analysis was performed in PALMIST (biophysics.swmed.edu/MBR/software.html) using the T-Jump mode [254]. A negative control experiment in which Soybean Trypsin Inhibitor (Worthington Biochemical Corp., Lakewood, NJ) was titrated into labeled PP1 under identical conditions showed only a weak trend in T-jump behavior (data not shown).

\section{Fluorescence polarization binding assay}

Purified PP1 $\alpha$ 7-330 was chemically linked to fluorescent probe using Alexa Fluor 488-N-hydroxysuccinimide ester (ThermoFisher Scientific) according to manufacturer protocol. 1-2 fluorophores were incorporated per one PP1 molecule. Reactions were carried in $35 \mu \mathrm{L}$ total volume with increasing concentrations of substrates (7xHis-Ska2/Ska1 or GST-Ska3 1-103) incubated overnight with $50 \mathrm{nM}$ fluorescently 
labeled PP1. Fluorescence polarization was subsequently measured on PHERAstar FS (BMG Labtech) and analyzed using OriginPro 7.5 software.

\section{In-vitro coupled transcription-translation and pull-down}

GST-tagged Ska3 or GST-Ska3/Ska1/Ska2 was incubated with Pierce ${ }^{\text {TM }}$ Glutathione Magnetic Beads (ThermoFisher Scientific) for 1 hour at $4^{\circ} \mathrm{C}$. Resin was subsequently washed three times with GF Buffer. ${ }^{35}$ S-methionine labeled FLAG-B55 subunits, obtained by 1 hour $\left(30^{\circ} \mathrm{C}\right)$ in vitro transcription and translation in $10 \mu \mathrm{L}$ of rabbit reticulocyte lysates (Promega), were diluted ten times in GF Buffer and incubated for 1 hour at room temperature with $10 \mu \mathrm{L}$ of magnetic beads coupled with GST-Ska constructs. After two $250 \mu \mathrm{L}$ washes with GF Buffer proteins were eluted with GF Buffer supplemented with $15 \mathrm{mM}$ Glutathione, subjected to SDS-PAGE, stained with Coomassie stain (Brilliant Blue R-250) and dried. After exposure of the phosphor screen, ${ }^{35} \mathrm{~S}$ radiation was detected by Storm Scanner.

\section{Phosphatase activity assay}

GST-Ndc80 ${ }^{\text {Bonsai }}$ WT was phosphorylated by Aurora A similarly to previously described method [226] and bound to Glutathione Sepharose 4 Fast Flow resin. Kinase and un-reacted $\left[{ }^{32} \mathrm{P}\right] \mathrm{ATP}$ was subsequently removed by rigorous washes. Untagged Ndc80 phosphosubstrate was removed from the resin by 1 hour cleavage of GST-tag with HRV 3C Protease. Phosphatase activity assays were performed on $200 \mathrm{nM} \mathrm{Ndc80}$ phosphosubstrate in phosphatase buffer (PBS, $1 \mathrm{mM} \mathrm{MnCl}_{2}, 1 \mathrm{mM}$ DTT) with $50 \mathrm{nM}$ hPP1 $\alpha$ 7-330 and $1 \mu \mathrm{M} 7 x H i s-S k a 2 / S k a 1$ or $1 \mu \mathrm{M}$ Ska complex. Over the time-course of the experiment $1 \mu \mathrm{L}$ samples were transferred to $1 \mathrm{~mL}$ of $30 \%$ activated charcoal in 50 $\mathrm{mM}$ phosphate buffer, $\mathrm{pH} 7.0$ and centrifuged. $500 \mu \mathrm{L}$ of the supernatant was mixed with $2 \mathrm{~mL}$ of scintillant and quantified in liquid scintillation counter. 


\section{Cell culture}

All cell experiments were conducted with HeLa cells. Parental HeLa cells were obtained from ATCC, Manassas, VA. HeLA Tet-On cells were obtained from Clontech, Mountain View, CA. HeLa cells stably expressing GFP-Histone H2B were provided by Geoff Wahl [255]. HeLa Ska1-GFP and HeLa Ska1 $\triangle$ MTBD ( $\Delta$ CTD) cell lines were obtained from Iain M Cheeseman [110]. All lines were routinely tested and found to be free of mycoplasma but were not further authenticated. All cell lines were grown in tissue culture dishes, culture flasks or chambered coverslips in $5 \% \mathrm{CO} 2$ at $37^{\circ} \mathrm{C}$ using complete DMEM media supplemented with $10 \%$ FBS, penicillin and streptomycin.

To synchronize HeLa cells, cultures were treated with $2 \mathrm{mM}$ thymidine for 18-24 $\mathrm{hr}$ and released into media containing $3.3 \mathrm{mM}$ nocodazole. Transient transfection for expression of plasmids was achieved using the Fugene 6 (Roche, Indianapolis, IN or Promega, Madison, WI or Mirus, Madison, WI) or Lipofectamine 3000 (Invitrogen, Carlsbad, CA) transfection reagent according to manufacturer's instructions. QuikChange Lightning site directed mutagenesis kit (Agilent technologies, Santa Clara, CA) was used to make point mutants of Ska1. Transfection of siRNA was done using Lipofectamine RNAi reagent (Invitrogen) according to manufacturer's instructions. SiGenome siRNA against Ska1, On-target plus SmartPool siRNA against Ska2 and Ska3 was obtained from DharmaconGE (Lafayette, $\mathrm{CO}$ ) and these were used at 25-50 nM final concentration.

To generate the stable cell lines, HeLa Tet-On cells were transfected with pTRE2 vectors expressing siRNA resistant GFP-Ska1 or GFP-Ska1 $\triangle$ CTD and selected with 300 $\mathrm{mg} / \mathrm{ml}$ hygromycin (Clontech). Further screening of the clones to obtain stable cells was done in the presence of $150 \mathrm{mg} / \mathrm{ml}$ hygromycin. 


\section{Live cell imaging}

HeLa H2B-GFP or HeLa Tet-On cells were grown in Nunc chambered coverslips (Thermo Sci. Inc., Waltham, MA). In some instances, to visualize DNA in HeLa cells, a cell permeable Hoechst dye (33342; Invitrogen) was used at 25-50 ng/ml. Time-lapse fluorescence images were collected every $5 \mathrm{~min}$ for $24-48 \mathrm{hr}$ using a Leica inverted microscope equipped with an environment chamber that controls temperature and $\mathrm{CO}_{2}$, 40X objective, an Evolve 512 Delta EMCCD camera, and Metamorph software (MDS Analytical Technologies, Sunnyvale, CA). Time-lapse videos displaying the elapsed time between consecutive frames were assembled using Metamorph or ImageJ software. The first time frame denoting onset of nuclear envelope breakdown (NEB), metaphase chromosome alignment and anaphase onset/mitotic exit was recorded in Microsoft Excel and the interval from NEB to metaphase (alignment time), metaphase to anaphase (metaphase duration) or NEB to anaphase onset/mitotic exit was calculated. For every cell, mitotic duration was calculated and the data were depicted as scatter plots with mean and SEM. Only cells expressing the indicated constructs (determined by mCherry expression) were counted to determine mitotic duration. The indicated proteins were tagged either in $\mathrm{N}$ terminus or $\mathrm{C}$ terminus and in both cases similar results were obtained by live cell imaging. Further, to be certain that the mCherry tag was not specifically influencing mitotic progression, the proteins were also tagged with GFP and again similar results were obtained. For clarity, only images and results obtained with mCherry-tagged proteins are presented. In scatter plots, each dot represents one cell; long horizontal line depicts mean and whiskers denote SEM. GraphPad Prism was used for statistical analysis. 


\section{Immunofluorescence}

HeLa cells were grown on glass coverslips or in Nunc chambered cover slides and treated as detailed in the figure legends. Cells were pre-extracted for 5 min using $1 \mathrm{X}$ PBS/PHEM solution containing 1\% Triton X 100 supplemented with phosphatase inhibitors (Okadaic acid at $1 \mathrm{mM}$ ). Cells were then fixed in 2 or $4 \%$ paraformaldehyde/PHEM solution supplemented with phosphatase inhibitors for $15 \mathrm{~min}$. Coverslips were washed in MBST, blocked in 20\% Boiled Normal goat/donkey serum or $2 \%$ Bovine serum albumin (BSA) for $1 \mathrm{hr}$, and incubated overnight at $4^{\circ} \mathrm{C}$ or $1 \mathrm{hr}$ at room temperature with primary antibodies. Samples were then incubated with secondary antibodies for $1 \mathrm{hr}$, stained with DNA dye, DAPI, and mounted using Vectashield (Vector Laboratories, Burlingame, CA). The following primary antibodies were used: ACA/CREST (Anti-Centromere antibodies from Antibody Inc, Davis, CA) and rabbit anti-Ska3 [137], rabbit anti-pMELT [143] and rabbit anti-Bub1 [256]. Secondary antibodies used were goat anti-rabbit or donkey anti-goat or goat anti-human antibodies conjugated to $\mathrm{Cy} 3$ or FITC (Jackson ImmunoResearch, West Grove, PA). The images were acquired using a Zeiss Axioplan II microscope equipped with a 100X objective (N.A. 1.4) or using 100X objective on Deltavision microscope (GE Healthcare, Pittsburgh, PA). Images were assembled using image $\mathbf{J}$ and CorelDRAW (Corel Corp, Ottawa, Canada). Quantification of the immunofluorescence images was done as described previously [137]. The graphs depict mean fluorescence value with SEM obtained from at least 10 cells in each condition. GraphPad Prism (Graphpad, La Jolla, CA) was used to determine statistical significance among groups. 


\section{Figures}

\section{Figure 3.1 Ska complex is required for PP1 recruitment to the kinetochore}

(A) HeLa cells were transfected with control or Ska3 siRNA at 50nM final concentration. Thirty hours after transfection immunofluorescence was done and PP1 at the kinetochore was quantified. Ska3 antibody staining shows efficiency of depletion. PP1 at kinetochores increases from prometaphase to metaphase. Ska3-depleted cells are inefficient in PP1 recruitment to kinetochores in both prometaphase and metaphase.

(B) HeLa cells were transfected with Mis12-GFP, Ska1-GFP or Mis12Ska1-GFP to increase Ska complex accumulation at kinetochores. Thirty-six hours after transfection, MG132 was added for $1 \mathrm{hr}$ to accumulate cells at metaphase. Immunofluorescence of PP1 at kinetochores was quantified. PP1 accumulates at kinetochores in Mis12Ska1GFPexpressing cells to a greater extent than in Mis12GFP- or Ska1GFP-expressing cells.

(C) In cells treated with $3.3 \mathrm{mM}$ nocodazole, PP1 accumulated to higher levels at kinetochores of cells expressing Mis12Ska1-GFP compared to cells expressing Mis12GFP or Ska1GFP. 


\section{Figure 3.1}

A

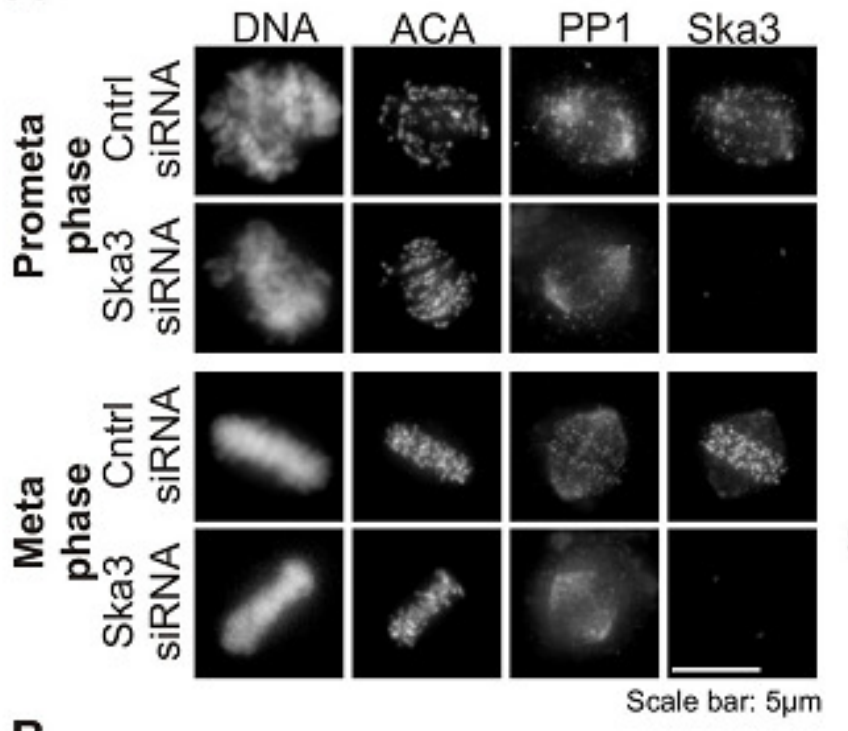

B
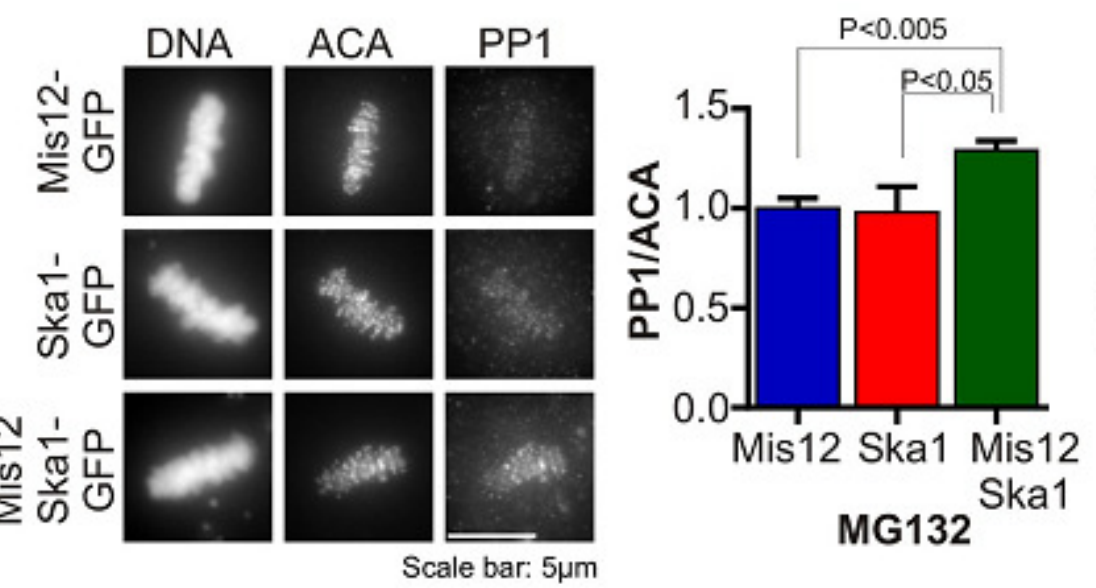

C

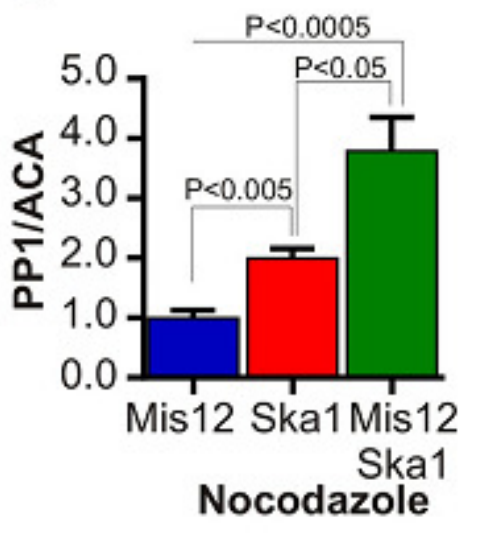




\section{Figure 3.2 Ska complex directly interacts with PP1 through Ska3.}

(A) Recombinant Ska3 was incubated with PP1-coated beads mixed with uncoated beads in various ratios and in vitro pull-down assay was performed. PP1 concentration is represented as total amount of PP1 immobilized on the beads per total volume of the pulldown reaction. Immunoblots show Ska3 co-pelleting with PP1 beads in concentration dependent manner, suggesting a direct interaction between the proteins.

(B) Densitometry quantification of Ska3 signal from the experiment depicted in panel (A) Number of replicates: 2; Error bars $=\mathrm{SD}$.

(C) Immunoblots showing the elution profile of recombinant PP1, Ska1/Ska2/Ska3 complex or PP1 preincubated with Ska1/Ska2/Ska3 complex run on a Superose 12 sizeexclusion column. 


\section{Figure 3.2}

A

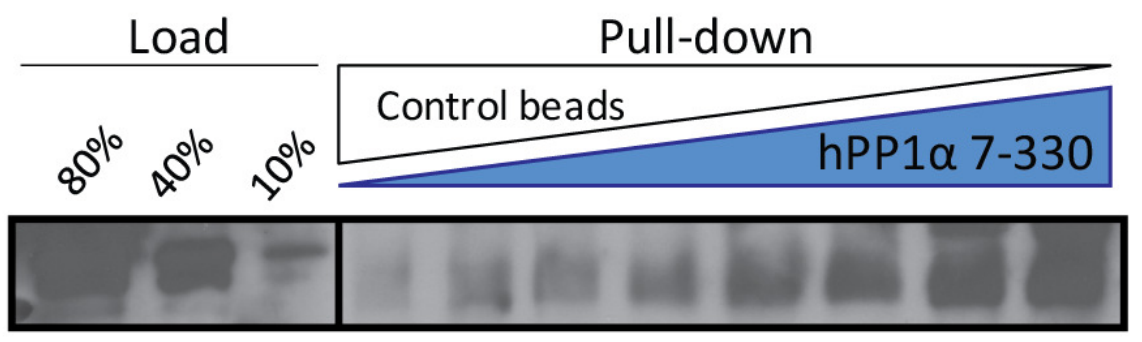

B

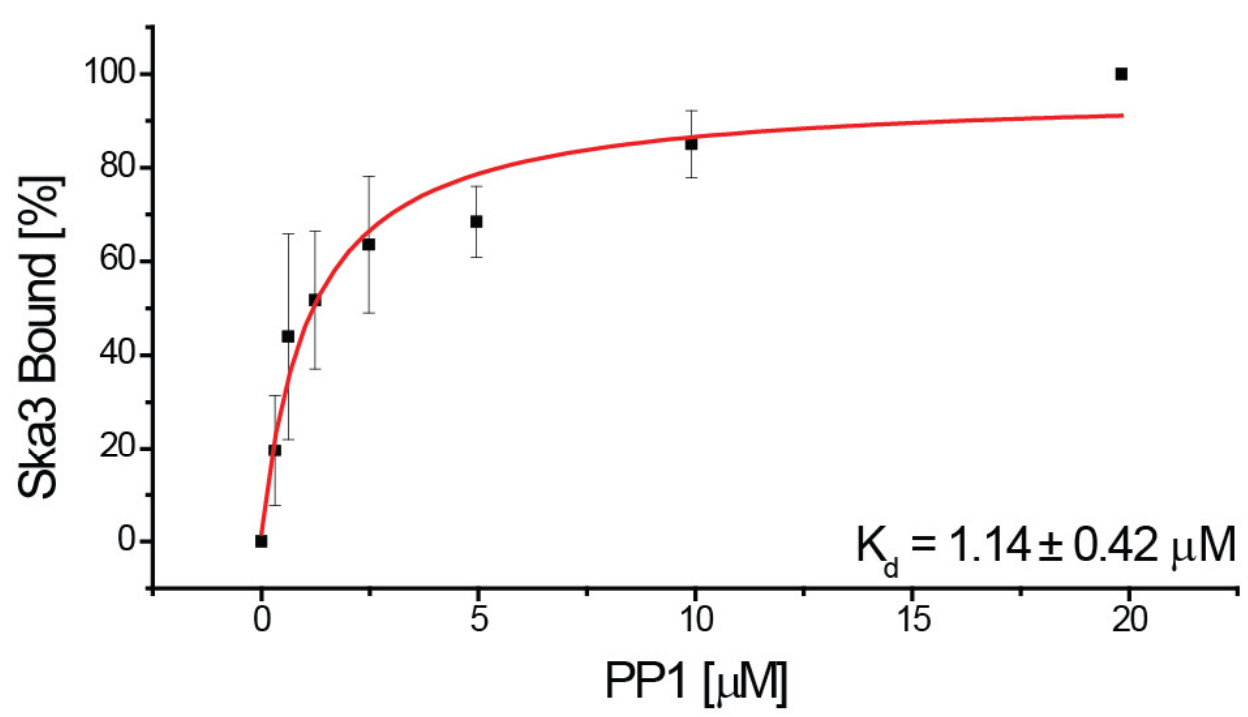

C

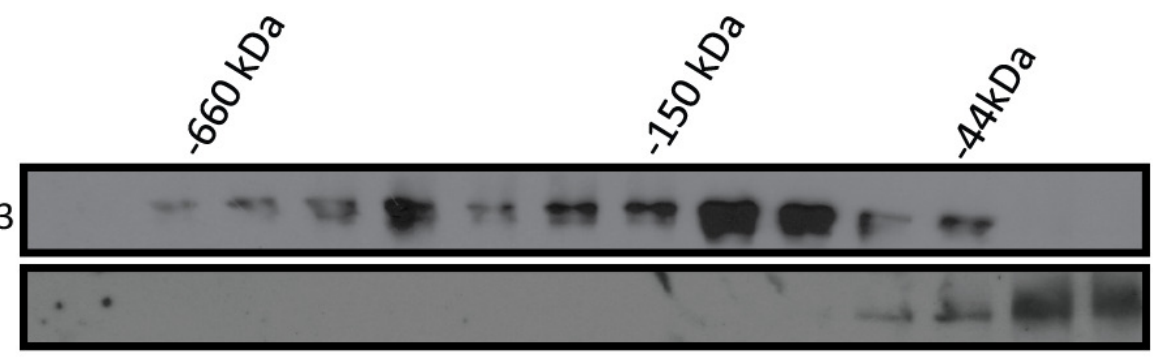

IB:

Ska3

PP1

Ska1/Ska2/Ska3

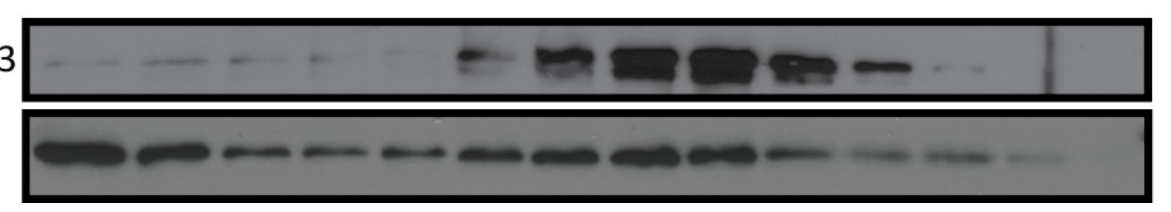

Ska3

+PPI

PP1 


\section{Figure 3.3 Ska3-PP1 interaction is multipartite.}

(A) PP1-bound CNBr-Activated Sepharose 4 Fast Flow resin was premixed with empty resin in various ratios prior to incubation with GST-Ska3 constructs. After one hour incubation at $4{ }^{\circ} \mathrm{C}$ resin was centrifuged and supernatant was collected and analyzed by Western blotting. GST-Ska3 was detected by anti-GST antibody. Ska3FL, 103-412 and 344-412 are depleted from supernatant with increasing concentrations of PP1.

(B) Immunoblots showing the elution profile of PP1 or PP1 preincubated with Ska3 ${ }^{1-343}$ or Ska1/Ska2/Ska3 ${ }^{1-343}$ complex run on a Superose 12 size-exclusion column. PP1* image of PP1 elution profile from Figure 3.2.C

(C) Graph representing fluorescence polarization of Alexa Fluor 488-PP1 incubated with increasing concentrations of GST-Ska3 ${ }^{1-103}$. GST-Ska3 ${ }^{1-103}$ concentration represented in $\log _{10}$ scale, insert: GST-Ska3 ${ }^{1-103}$ concentration represented in linear scale. Number of replicates: 3; Error bars $=\mathrm{SD}$. 


\section{Figure 3.3}
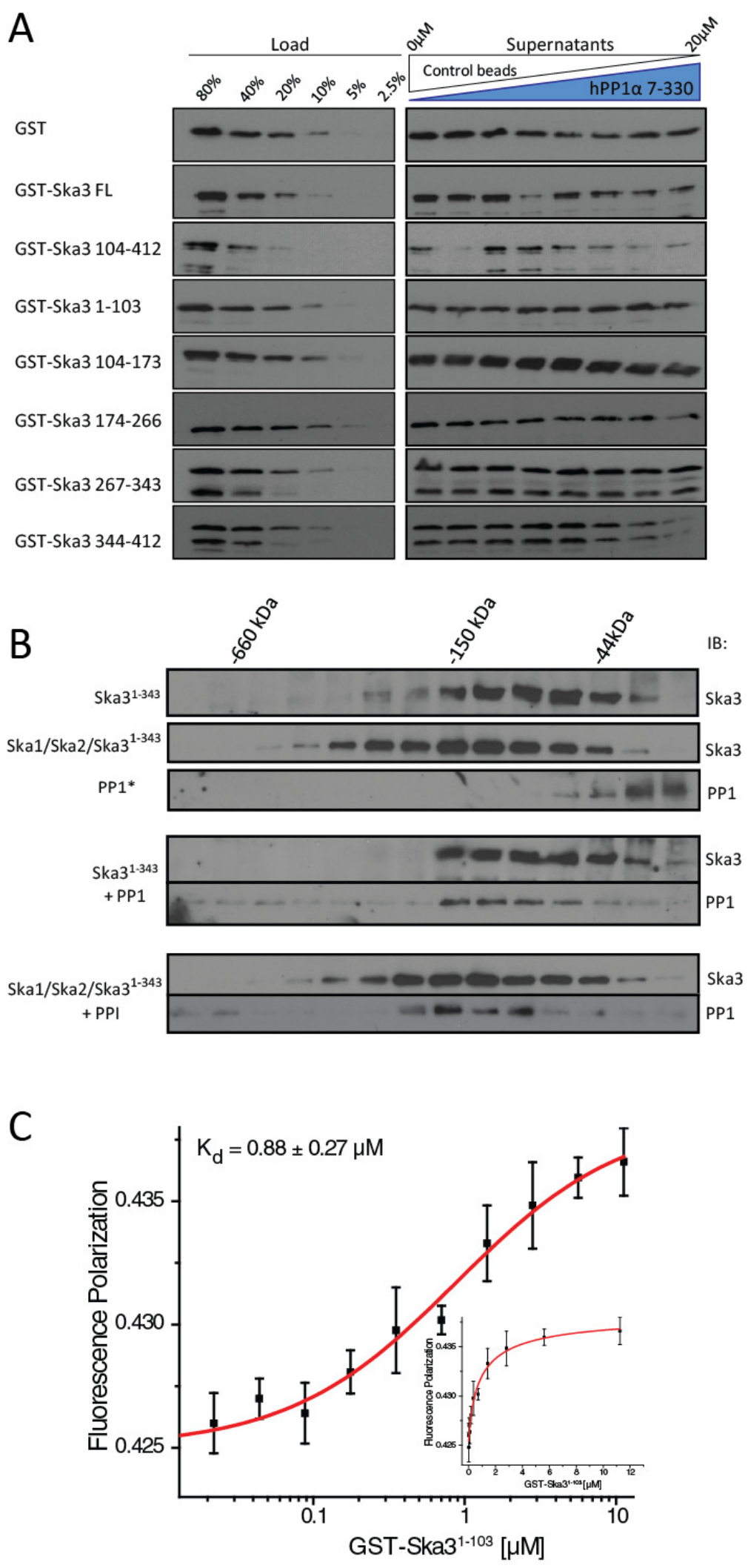

Pawel Janczyk, unpublished data 


\section{Figure 3.4 Ska1 CTD directly binds to PP1.}

(A) Release of ${ }^{32} \mathrm{P}$ phosphate groups by PP1 from Aurora-phosphorylated Ndc $80^{\text {Bonsai }}$ was measured by liquid scintillation counter over the course of the reaction. Addition of both Ska1/Ska2 (red) and Ska1/Ska2/Ska3 (green) was able to similarly increase ${ }^{32} \mathrm{P}$ release rates. This indicates that Ska1/Ska2 is sufficient to stimulate PP1 phosphatase activity.

(B) Microscale thermophoresis (MST) was done to analyze the direct binding interaction between PP1 and the Ska1 CTD proteins purified from bacteria. The top panel shows thermophoretic time traces of 16 samples in three independent experiments. The middle panel shows the T-Jump data (circles) and the fit to the data (line). The residuals between the data and the fit line are indicated in the bottom panel. The $\mathrm{K}_{\mathrm{d}}$ of PP1 binding to the Ska1 CTD was calculated to be $1.5 \mathrm{mM} . \mathrm{F}_{\mathrm{n}}$ represents ratio (expressed in per-mille units) of the fluorescence readings in the time traces as measured just after (pink region, top panel) and before (blue region, top panel) activation of the MST laser; $\Delta F_{n}$ is calculated by subtracting the refined $F_{n}$ of the free PP1 from all $F_{n}$ values and thus represents the change in T-Jump response as a function of ligand concentration. This part was rendered using the program GUSSI [257].

(C) Immunoblots showing the elution profile of PP1, PP1 incubated with Ska1FL/Ska2/Ska3 ${ }^{1-343}$ (blue) or PP1 incubated with Ska1 $\Delta$ CTD/Ska2/Ska3 ${ }^{1-343}$ (orange) run on a Superose 12 size-exclusion column. Ska complexes run in the same fraction in the absence of PP1 (not shown). Densitometry quantifications of PP1 signal in fractions eluted from size-exclusion column shows a reduction in binding to Ska complex lacking the Ska1 CTD. 


\section{Figure 3.4}

A

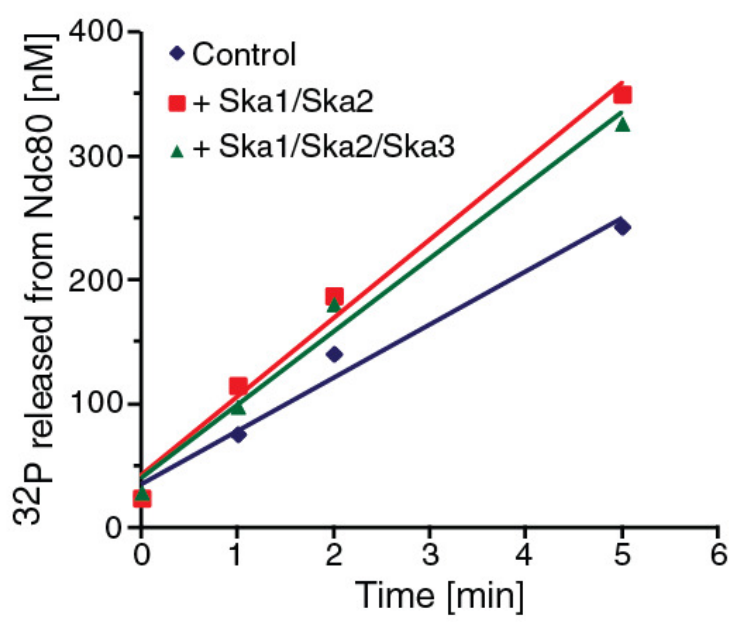

C

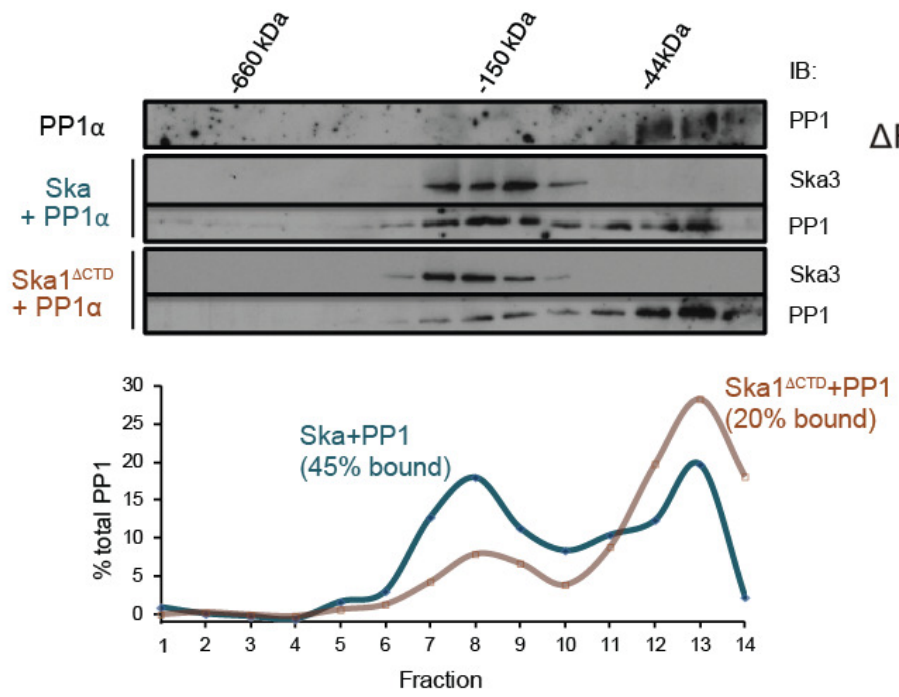

B

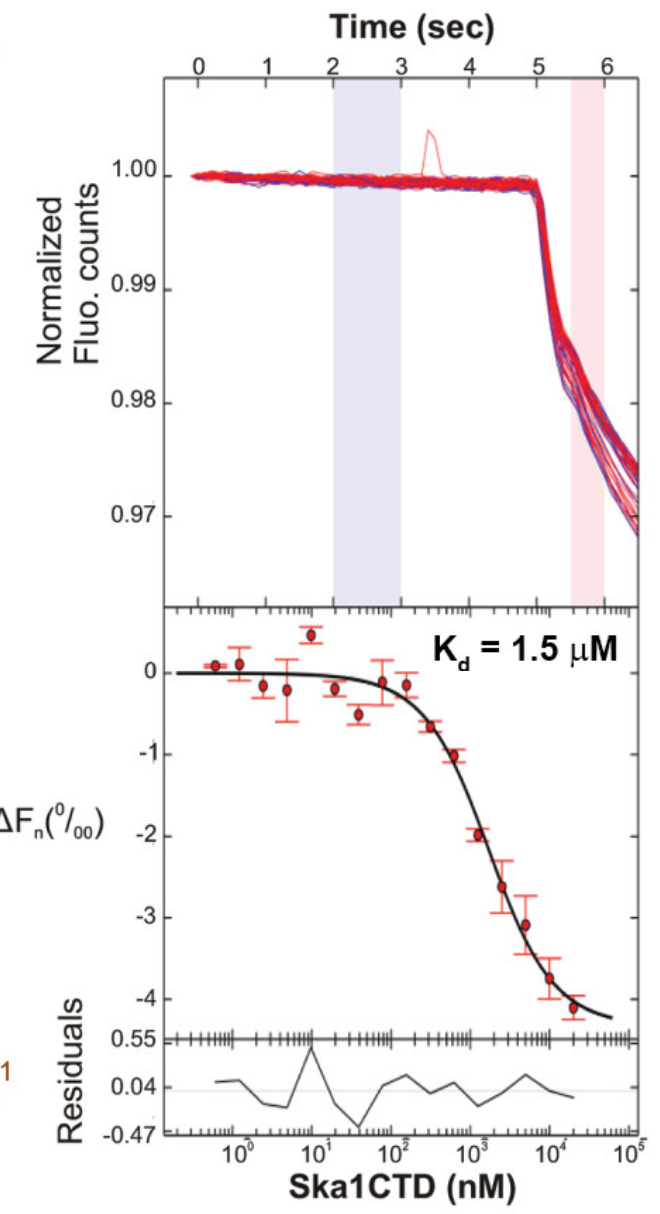

Pawel Janczyk $(A, C)$ and Sushama Sivakumar (B),

(A) - unpublished data, (B, C) - Sivakumar et al., 2016 
Figure 3.5 Cells expressing Ska1 $\triangle \mathrm{CTD}$ recruit less PP1 and accumulate more Knl1 phosphoepitope and Bub1 protein at kinetochores.

HeLa GFP-Ska1 and HeLa GFP-Ska1 $\Delta$ CTD cells were grown on chambered cover slides. Doxycyclin was added to induce transgene expression. Cells were transfected with Ska1 siRNA, and thymidine was added for $18-24 \mathrm{hr}$ to synchronize cells. Cells were released from thymidine and arrested in mitosis using $3.3 \mathrm{mM}$ nocodazole. Immunofluorescence was done to detect PP1, pMELT, Bub1 at the kinetochore.

(A) Image panel showing PP1 localization at the kinetochore in GFP-Ska1 and GFPSka1 $\Delta$ CTD cells. The graph depicts the decrease in PP1 localization in GFP-Ska1 $\Delta C T D$ cells compared to GFP-Ska1 cells both with and without depletion of endogenous Ska1. (B) Images show localization of antibody (pMELT) to a phosphoepitope on Knl1 (pT875) at kinetochores in GFP-Ska1 and GFP-Ska1 $\triangle$ CTD cells. The graph shows that cells expressing GFP-Ska1 $\triangle$ CTD have increased pMELT signals at kinetochores compared to GFP-Ska1-expressing cells.

(C) Images show the levels of Bub1 protein at kinetochores in GFP-Ska1 and GFPSka1 $\Delta$ CTD cells. Quantification shows that GFP-Ska1 $\Delta$ CTD accumulates more Bub1 at kinetochores compared to GFP-Ska1 cells. 
Figure 3.5

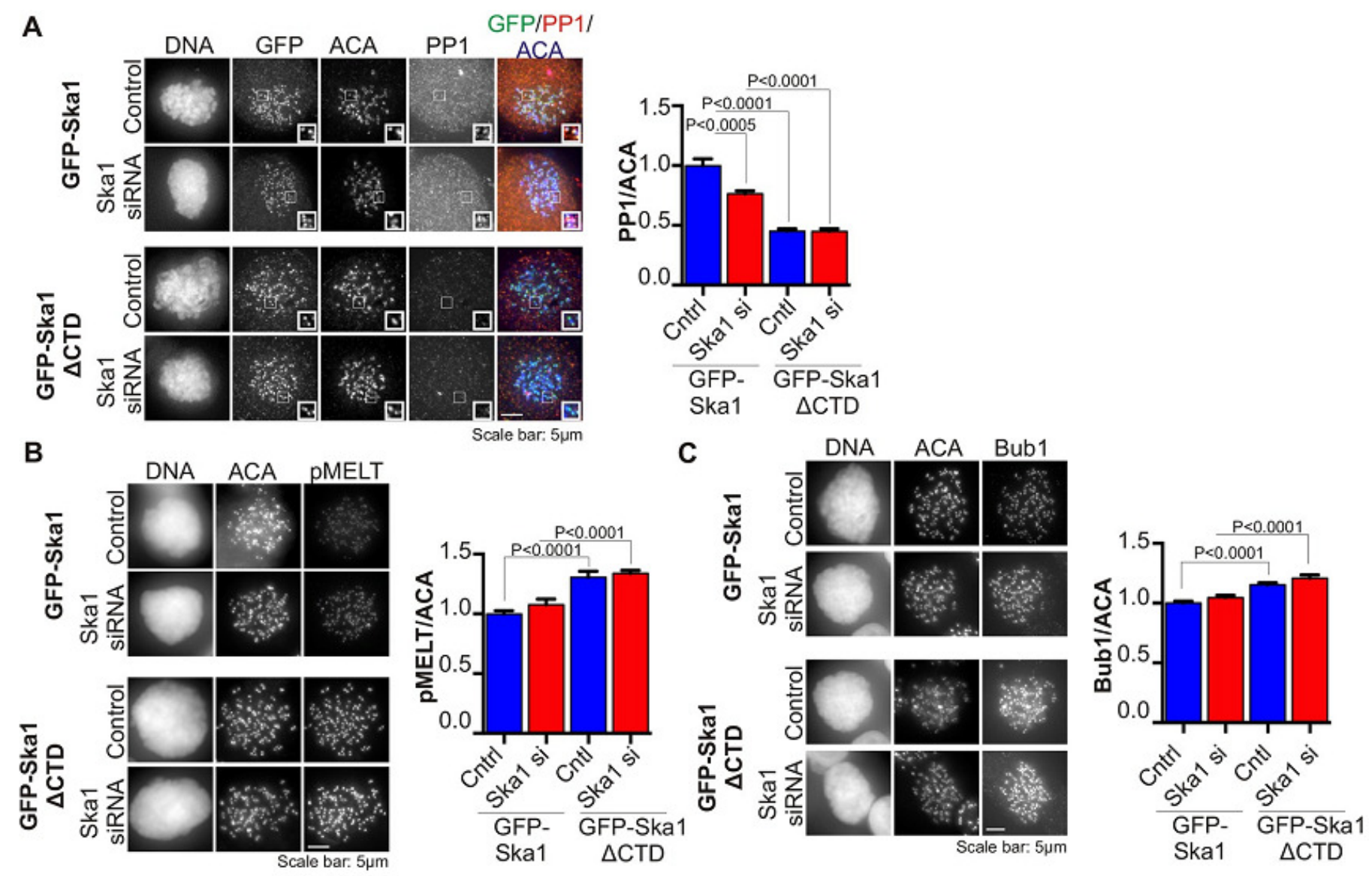

Sushama Sivakumar, Sivakumar et al., 2016 


\section{Figure 3.6 Expression of Ska1 $\triangle \mathrm{CTD}$ fused directly to PP1 but not phosphatase-dead PP1 (pdPP1) rescues phenotypes caused by Ska1 depletion.}

(A) HeLa cells were transfected with plasmids to express mCherry-Ska1, mCherry-PP1, mCherry-Ska1 $\Delta$ CTD, and mCherry-Ska1 $\Delta$ CTD fused to PP1 (mCherry-Ska1 $\Delta C T D P P 1)$. Endogenous Ska1 was depleted using Ska1 siRNA. 25 ng/ml Hoechst 33342 was added to visualize DNA. Cells were then imaged by time-lapse microscopy, and \% of mitotic cells arrested in metaphase was plotted. Expression of siRNA-resistant mCherry-Ska1 rescued metaphase arrest caused by Ska1 depletion while expression of mCherry-PP1 or mCherry-Ska1 $\Delta$ CTD did not. Expression of Ska1 $\Delta$ CTDPP1 fusion in Ska1 depleted cells completely rescued metaphase arrest.

(B) The interval from NEB to anaphase onset is plotted for cells that progressed to anaphase while expressing Ska1 constructs without or with depletion of endogenous Ska1. As expected, Ska1 $\triangle$ CTD-expression showed a dominant negative effect delaying mitotic progression in control cells not depleted of endogenous Ska1. Expression of the fusion, Ska1 $\triangle$ CTDPP1, caused no delay. When endogenous Ska1 was depleted, 38\% of cells arrested at metaphase and did not progress to anaphase (Figure 3.5.A). The rest showed delayed progression from NEB to anaphase with an average of $110 \mathrm{~min}$ compared to control cells (35 min). As expected, the delay was rescued by expression of mCherry-Ska1 but was not rescued by expression of mCherry-PP1 or mCherrySka1 $\Delta$ CTD. Expression of Ska1 $\triangle$ CTDPP1 showed significant rescue of the delay cause by Ska1 depletion with an average time from NEB to anaphase of $62 \mathrm{~min}$.

(C) HeLa cells were transfected with the indicated plasmids and then treated with mock or Ska1 siRNA. Expression of Ska1 $\triangle \mathrm{CTD}$ fused to a phosphatase dead PP1 (Ska1 $\triangle \mathrm{CTDpdPP} 1)$ failed to rescue Ska1 depletion. Indeed, expression of phosphatase 
dead fusion, on its own, induced a potent metaphase arrest phenotype in cells not depleted of endogenous Ska1. Moreover, it exacerbated the metaphase arrest in cells depleted of Ska1.

(D) Ska1 $\Delta$ CTDpdPP1 causes a longer delay to anaphase onset than Ska1 $\Delta$ CTD even without depletion of endogenous Ska1.

(E) Chromosome alignment is delayed in Ska1 depleted cells and this is recapitulated by expression of Ska1 $\triangle$ CTDpdPP1 without endogenous Ska1 depletion. Delays in chromosome alignment are not observed upon expression of Ska1 $\Delta$ CTDPP1 fusion. 


\section{Figure 3.6}

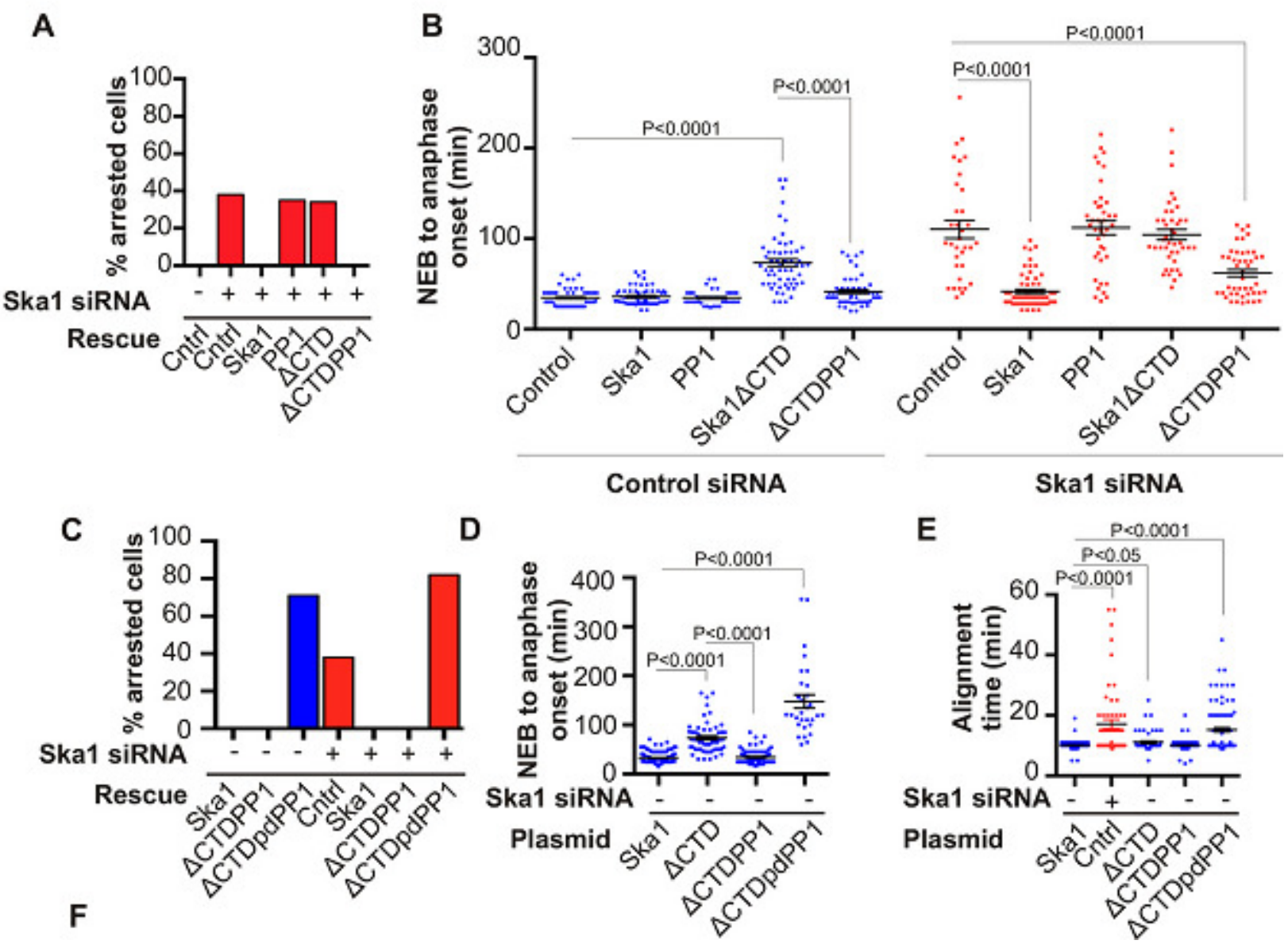

Sushama Sivakumar, Sivakumar et al., 2016 
Figure 3.7 Hypothetical model for dynamic balance of Mps1 kinase and PP1 phosphatase activities during mitotic progression.

Photobleaching studies have shown that Mps1, Ska, and PP1 all exhibit high turnover at kinetochores with a residence times of a few seconds [136,197,247]. Before microtubule attachment, Mps1 concentration at kinetochores remains high due to interaction with the $\mathrm{CH}$ domains of the Ndc80 complex. Correspondingly, Ska-PP1 concentrations are low because of the paucity of microtubules. The high Mps1 and low PP1 concentrations maintain high phosphorylation Mps1 substrates, Knl1 and Bub1. Microtubules compete with Mps 1 for binding to the $\mathrm{CH}$ domains of the $\mathrm{Ndc} 80$ complex, resulting in depletion of Mps1. The binding of Ska to microtubule protofilaments increases PP1 concentration. High Ska-PP1 and low Mps1 result in dephosphorylation of substrates, promoting release of Bub1-Bub3 and Mad1-Mad2 complexes. Diminished checkpoint signaling due to release of Bub1-Bub3 and Mad1-Mad2 from kinetochores promotes anaphase onset and mitotic exit. As the role and nature of the Ska-B55 interaction is unclear, it is not represented in this model.

Reproduced from [220] 
Figure 3.7
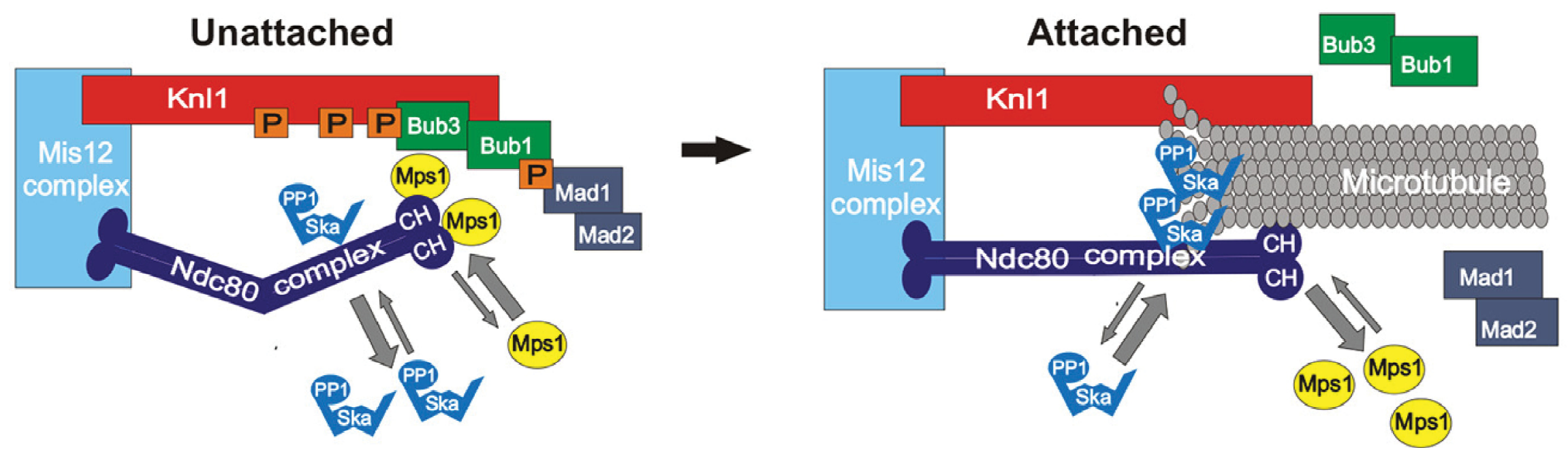


\section{Supplemental Figure 3.1}

(A) GST-Ska complex was purified from bacteria and immobilized on glutathione beads. In vitro translated PP1 $\alpha$ and $\mathrm{B} 55$ isoforms were added to the resin and binding assays were performed. B55 $\beta$ and B55 $\gamma$ bound strongly with GST-Ska complex, while PP1 $\alpha$ and B55 $\alpha$ did not show a strong binding. (Top: Coomassie stained polyacrylamide gel, Bottom: ${ }^{35} \mathrm{~S}$ autoradiography)

(B) In vitro binding reactions were performed similarly as in (A) utilizing low concentrations of immobilized GST-Ska complex and in vitro translated B55 isoforms alone or premixed with in vitro translated PP1 $\alpha$. Interaction with the Ska complex with B55 $\alpha$ (top panel), B55 $\beta$ (middle panel) was enhanced by PP1 $\alpha$. Ska-B55 $\gamma$ (bottom panel) binding was not increased by presence of PP1 $\alpha$ 
Supplemental Figure 3.1

A

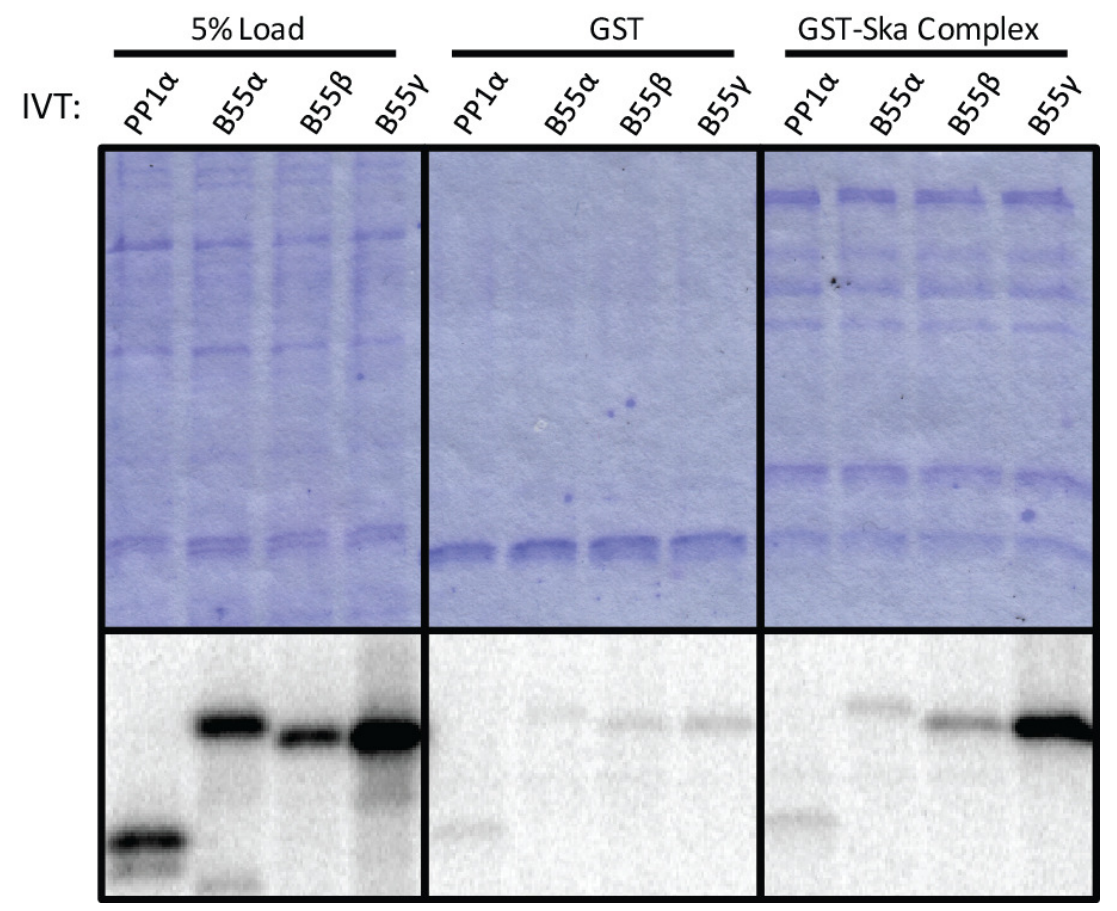


B
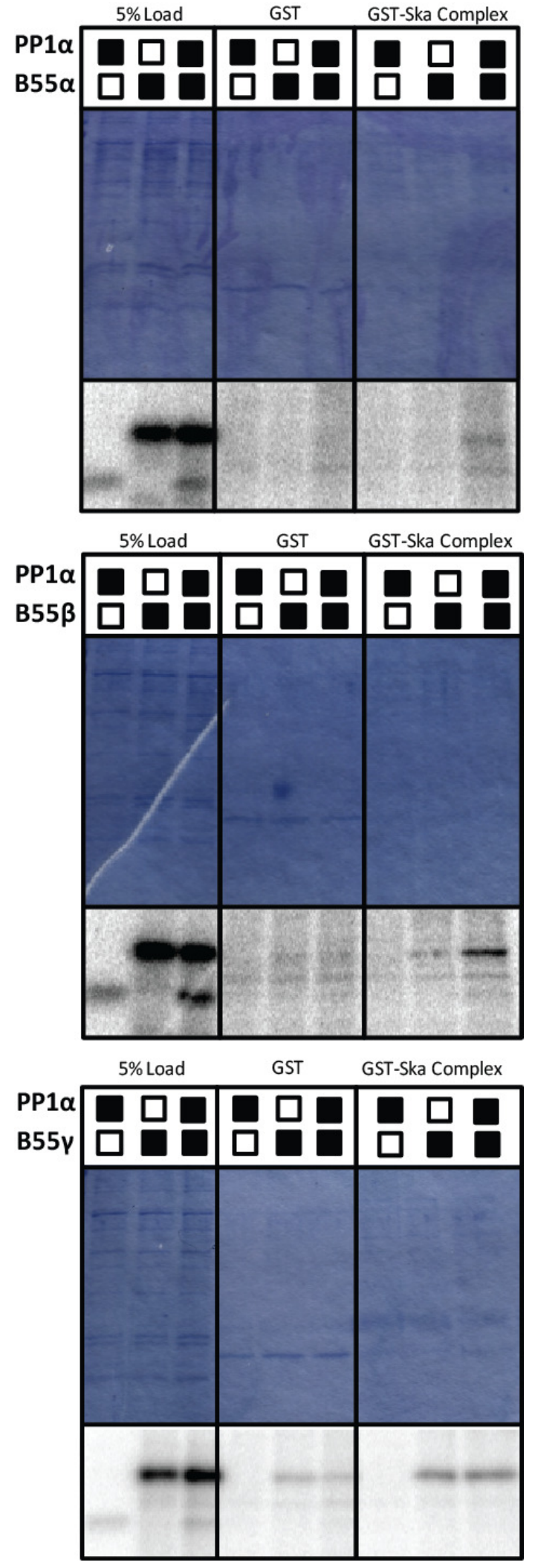

Pawel Janczyk, unpublished data 


\section{Acknowledgements}

We thank Iain M Cheeseman for plasmids and HeLa cell lines expressing GFPSka1 and HeLa GFP-Ska1 $\Delta$ CTD. We thank Geoff Wahl for the HeLa cell line expressing GFP-Histone H2B. We thank Wolfgang Peti for PP1 plasmid. We thank David L Brautigan for PP1 antibody, plasmids, and for useful advice. We also thank members of the Yu, Gorbsky, and Stukenberg laboratories for help with experiments and useful suggestions. We would especially like to thank Dr. Haishan Gao in Dr. Yu's laboratory for his help with protein purification. This study was supported by funding from National Institutes of Health, grant number RO1GM111731 to GJG and grant number R01GM081576 to PTS, from the McCasland Foundation to GJG, and from the Clayton foundation to HY. 


\section{Chapter IV \\ General discussion and future perspectives}

Our understanding of kinetochore functions in coupling microtubule depolymerization has come a long way over the last decade. Formation of stable end-on attachments is a crucial step in mitosis, required for chromosome movements driven by microtubule depolymerization. The discovery of the Ndc80 complex and characterization of its microtubule binding properties shed a light on how kinetochores attach microtubules. Aurora B-dependent phosphorylation of the Ndc80 tail has been shown to regulate the Ndc80 binding to microtubules. This is an astonishing ability that explains how the attachment stability is regulated by the kinetochore and additionally, provides a mechanism for correction of the erroneous ones. However, it has not been understood how the kinetochore would recognize mature attachments to allow for checkpoint silencing and chromosomal segregation. Recent studies have shown that the checkpoint kinase, Mps1, is displaced from Ndc80 when microtubules are attached. This discovery provided an insight into the steps required for the attachment status recognition. However, the Ska complex was shown to act downstream of Mps1 and its depletion results in the checkpoint-dependent arrest with all chromosomes aligned and under tension. Interestingly, Ska itself has been shown to bind to microtubules and it likely contributes to the attachment stabilization. It was previously suggested that Ska is a direct coupler for depolymerization driven movements as it is able to track depolymerizing microtubule ends and it confers that ability to Ndc80. This property, together with Ska functions in checkpoint silencing, suggested that the Ska complex might be a key component in recognizing microtubule attachments and driving the mitotic exit. However, it was unclear how Ska would recognize mature attachments. It was also 
unknown how Ska would trigger SAC silencing. Results presented in this dissertation provide answers to those questions as described in detail in Chapter II and Chapter III, and summarized below.

\section{Summary}

In chapter II we investigated the functions of the N-terminal tail of Ndc80. Our results suggest that main function of the $\mathrm{Ndc} 80$ tail, besides microtubule binding, is to facilitate clustering of the Ndc80 molecules along microtubule protofilaments. We show that cells expressing the $\mathrm{Ndc} 80$ mutant that could bind microtubules with similar affinity as wild-type protein, but was deficient in cluster formation $\left(\mathrm{Ndc} 80^{+4 \mathrm{CT}}\right)$ could align chromosomes to the metaphase plate. However, those cells remained arrested in metaphase, suggesting that although the clustering of Ndc80 molecules on microtubules is dispensable for chromosome congression, it is required for SAC silencing and mitotic exit. We discover that this mutant was unable to efficiently recruit the Ska complex to the kinetochores, arguing that the ability to cluster on microtubules might be correlated with novel mitotic functions of the Ndc80 tail. By means of negative stain electron tomography we discovered novel structures on microtubules that can be observed in presence of Ska and Ndc80, but were not visible in absence of $\mathrm{Ndc} 80$ or when $\mathrm{Ndc} 80^{+4 \mathrm{CT}}$ mutant was used. It strongly suggests that Ska and Ndc80 form a supercomplex on microtubules that appears to be dependent on Ndc80 clustering. However, we cannot exclude the possibility that Ska recognizes charged residues on the Ndc80 tail and the clusters are not essential for binding. Altogether, these results provide an insight to novel function of the Ndc80 N-terminal tail in the kinetochore recruitment of the Ska complex. It additionally provides a mechanism for the recognition of mature attachments by the Ska complex, as the Ndc80 cluster can preferably be formed on aligned kinetochores with reduced Aurora B activity. 
In Chapter III of this dissertation we investigated the functions of the Ska complex in the process of spindle checkpoint silencing. We discovered that the Ska complex directly binds and recruits PP1 to the kinetochore. The Ska complex showed multipartite association with PP1 through Ska3 and the C-terminal domain of Ska1. Ska3 is an essential component of the Ska complex, with its N-termini required for complex oligomerization and C-termini implicated in kinetochore localization. Unfortunately, we were unable to generate a mutant that would separate those functions from the PP1 binding. On the other hand, investigation of Ska1 CTD demonstrated its crucial role in PP1 recruitment to the kinetochore. Deletion of this domain was shown to result in a phenotype virtually identical to one when Ska was completely depleted for the cells, which was previously attributed to the microtubule binding properties of the Ska1 CTD (also named MTBD). Interestingly, we show that the expression of the Skal with CTD replaced by PP1 rescues nearly all the mitotic phenotypes observed upon Ska depletion and this rescue is dependent on the phosphatase activity of the chimera. This result strongly suggested that, rather than serving as a mechanical coupler between kinetochores and microtubules, the Ska complex plays a critical role in the recruitment of PP1 to oppose mitotic kinases that are responsible for destabilization of kinetochore-microtubule attachments and signaling the spindle checkpoint.

Altogether, these results provide an insight into the processes of the attachment maturation and simultaneously, recognition of the mature attachments. Based on them, we would like to propose a model in which upon alignment, Aurora B activity on the outer kinetochore is diminished by physical separation due to increased interkinetochore distances. This would result in the decreased phosphorylation of the Ndc80 tail allowing for clustering of $\mathrm{Ndc} 80$ molecules along microtubule protofilaments, and thus, recruitment of Ska to Ndc80 clusters. Kinetochore-associated Ska would in turn localize 
PP1 phosphatase to the kinetochore allowing for dephosphorylation of mitotic phosphosubstrates, likely including Ndc80 (Figure 4.1). This could result in a positive feedback loop that stabilizes the attachments, thus recruiting more PP1 and priming the cell for rapid transition to anaphase upon establishment of the last mature kinetochoremicrotubule attachment. 


\section{Figure 4.1}

High Aurora B activity Transient attachment SAC"on"

Low Aurora B activity Low PP1 activity Stable attachment SAC"on"

Low Aurora B activity High PP1 activity Mature attachment SAC "off"

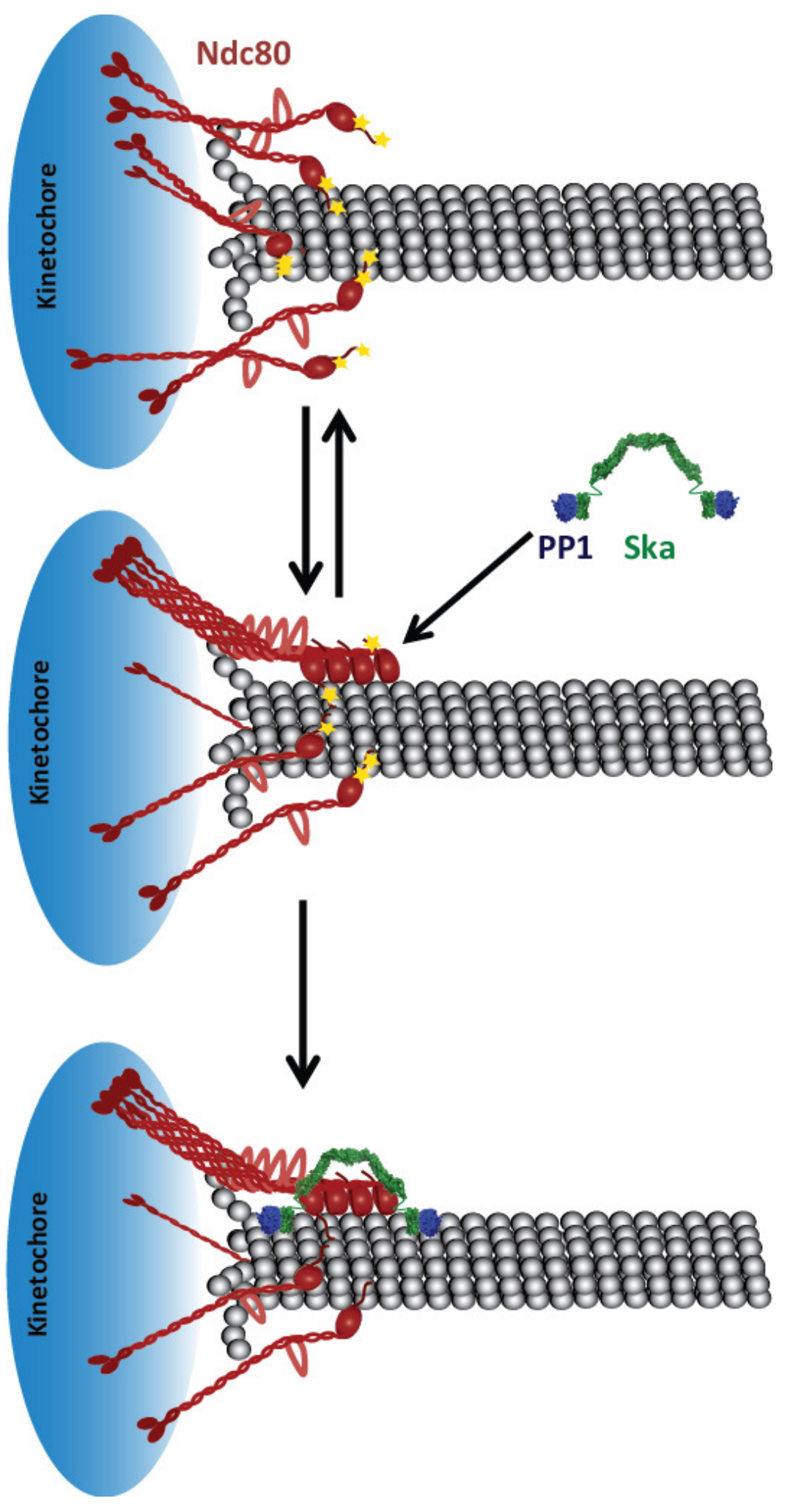




\section{Future perspectives}

\section{Identification of the components of the V-shaped structures}

Using a negative stain electron tomography, we identified novel V-shaped structures that are formed on microtubules. They are formed only when Ska and the Ndc80 complexes are both incubated with the microtubules. No such structures were observed when the individual components were incubated with the protofilament. Unfortunately, our current data does not provide sufficient evidence that both complexes are present in the V-shaped structures. We speculate that the Ska complex is actually present in this system as both shape and size of the Ska crystallographic structure [144] are reflected in the V-shaped design. However, up to date we only have a single evidence of tomographic reconstitution that the $\mathrm{Ndc} 80$ clusters co-localize within the V-shapes. The main drawback so far is the insufficient quality/resolution of the reconstructions that did not allow for the separation of structural components of the V-shapes. Additionally, $\mathrm{Ndc} 80^{\text {Bonsai }}$ used for the experiments is $17 \mathrm{~nm}$ long, but in our reconstructions only $8-10$ $\mathrm{nm}$ is visibly protruding away from the microtubules. In order to improve the quality of the tomographic reconstructions and confirm the presence of both complexes building this novel framework, one could implement multiple approaches. In order to improve the quality of the tomograms, Ndc80 molecules should be more noticeable. So far, purification of the human full length Ndc80 complex is challenging and obtained amount are insufficient for our studies. However, $\mathrm{Ndc} 80^{\text {Broccoli }}[110]$ that contains the majority of the Ndc80's and Nuf2's elongated coiled coil may be used instead of Ndc80 ${ }^{\text {Bonsai }}$. This way, one would extend the length of the protruding molecules which may make them more visible in the reconstructions. Alternatively, C-terminally GFP-tagged Nuf2, a part of Ndc $80^{\text {Broccoli }}$ construct, could be used. The GFP-tag will increase the electron density on the far end of the molecule and it will not interfere with the C-terminal end of Ndc80 
$\mathrm{N}$-terminus that is responsible for clustering the molecules and recruitment of Ska. In our preliminary experiments we observed that the $\mathrm{Ndc} 80^{\text {Broccoli }}$ molecules were protruding further away from the microtubule lattice. Additional density was even more noticeable when we used a GFP-tagged construct, as the globular domain of the GFP tag at the end of the coiled-coil was clearly visible in our micrographs. Additionally, the use of Ni-NTA gold nanoparticles could help in the identification of the protein of interest within the supercomplex as the nanoparticles may be conjugated with His-tagged protein and mark its position within the $\mathrm{V}$-shaped cluster. By this approach we might not only confirm the presence of both complexes, but also differentiate between complexes' subunits.

\section{Characterization of the requirements for the assembly of the Ndc80-Ska supercomplex and subsequent recruitment of PP1.}

Additional structural studies can be performed to analyze the requirements for the formation of the Ska-Ndc80 complex on the microtubules. In this dissertation, I analyze the role of Ndc80. It would be interesting to investigate how different components of the Ska complex influence formation of supercomplex. There are multiple functional components of the Ska that I mentioned in this work that may be crucial to broaden our knowledge about kinetochore-microtubule attachment maturation. For example, the unstructured C-terminus of the Ska3 has been shown to be important for both microtubule binding and kinetochore localization [144]. Additionally, the C-terminal domain of Ska1 contains a MTBD, although depletion of that domain does not mislocalize Ska from kinetochores [144]. Finally, the dimerization of the Ska complex was previously implicated in the mitotic functions [144] and the Ska3 ${ }^{\mathrm{F} 7 \mathrm{R} / \mathrm{L} 11 \mathrm{R}}$ mutant was previously shown to form a complex with different structural architecture than the wild-type. It would be interesting to analyze how disruptions within mentioned domains influence 
binding between subunits of the supercomplex and to analyze the changes in the structural arrangements of the V-shapes.

Based on the results presented in this dissertation, I hypothesize that the V-shapes formed by Ndc80 and Ska should facilitate the recruitment of the PP1 to the kinetochore. However, additional studies should be performed to confirm the existence of the complex composed of $\underline{\mathrm{N} d c 80}$, $\underline{\mathrm{S} k a}$ and $\underline{\mathrm{P}} 1$ (NSP), e.g. by electron tomography characterization similar to ones described above.

\section{Ndc80-dependent Ska recruitment in the absence of microtubules}

It is currently believed that the main function of the $\mathrm{Ndc} 80$ tail is to contribute to the microtubule binding of the Ndc80 complex. In this dissertation, I described a novel function of the tail which is the recruitment of the Ska complex to the kinetochores. I have also shown that this recruitment is dependent on the positively charged residues within the C-terminal region of the tail. We hypothesize that clustering of $\mathrm{Ndc} 80$ molecules is required for Ska recruitment. Interestingly, the Ska recruitment by the Ndc80 tail is not dependent on the microtubules, as inhibition of the Aurora B kinase activity allows for the Ska kinetochore-localization in the presence of nocodazole. Based on this result, we speculate that Ska may be involved in clustering of Ndc80 or clustering is very convincing in vitro artifact. We speculate that Ska clusters Ndc80 molecules on the kinetochores in the absence of microtubules, a function that is abrogated by the Aurora B activity. One could test this hypothesis in vivo by utilizing the nonphosphorylatable mutant of $\mathrm{Ndc} 80\left(\mathrm{Ndc} 80^{9 \mathrm{~A}}\right)$. The cells expressing this mutant should allow for Ska recruitment in the presence of nocodazole. As Ska is also phosphorylated by Aurora B, it is likely that non-phosphorylatable mutant of Ska would have to be used in addition. On the other hand, cells expressing a phospho-mimetic mutant of Ndc80 
$\left(\mathrm{Ndc} 80^{9 \mathrm{D}}\right)$ should be deficient in the recruitment of the Ska complex to the kinetochores, even when treated with Aurora B inhibitor.

In vitro investigation of the Ska-induced clustering of Ndc80 molecules might prove to be difficult. So far, the direct interaction between Ska and Ndc80 in the absence of microtubules has not been shown. It is likely that it requires a lawn-type immobilization of the $\mathrm{Ndc} 80$ in order to position $\mathrm{CH}$ domains in close proximity to stimulate the binding.

Another experiment that could be used to confirm the hypothesis involves the ectopic targeting to the $\mathrm{LacO}$ arrays in $\mathrm{U} 2 \mathrm{OS}$ cells. $\mathrm{LacO}$ arrays were previously shown to allow for formation of synthetic kinetochore in budding yeast [256]. The arrays could serve as a matrix to bring LacI-tagged Ndc80 molecules close enough to facilitate the clustering that may be used in future studies. There are disadvantages of this assay, as it is unknown if LacO array would mimic the kinetochore in terms of Ndc80 arrangement. Additionally, it is not known if other kinetochore components is essential for Ndc80 clustering in the absence of the microtubules as, for example the Mis 12 complex was shown to stimulate the $\mathrm{Ndc} 80$ binding to microtubules and to modify its structural arrangement [98].

\section{NSP as a microtubule coupler required for chromosomal movements}

Current model for the chromosomal movements places Ska and Ndc80 complexes in the central spot as the couplers between the kinetochores and the microtubules. Ska helps Ndc80 track depolymerizing plus-ends of microtubules. We show that the domain responsible for this function (Ska1 CTD) [110] also facilitates the recruitment of PP1 to the kinetochores. Phenotypes observed in cells expressing Ska1 $\Delta$ CTD can be rescued by Ska1CTD-PP1 chimera suggesting that microtubule binding is dispensable for Ska 
functions. It is tempting to hypothesize that the clusters of Ndc80 molecules are recognized by (or induced by) the Ska complex on the depolymerizing microtubule, independent of Ska microtubule binding functions, and the resulting structure is the direct coupler for depolymerization driven movements. However, it is also likely that the main function of the Ndc80 clusters is to recruit PP1 to silence spindle checkpoint.

The Ska complex has also been shown to be required for oscillations of the chromosomes aligned at the metaphase plate [110]. This process involves microtubule polymerization on one kinetochore, the simultaneous depolymerization on its sister kinetochore and coordinated switches between those states. EB1, protein specifically associating with the polymerizing microtubule ends, was recently implicated in the recruitment of the Aurora B kinase to the inner-centromere. Interestingly, Aurora B activity is correlated with EB1 localization on the oscillating chromosomes [224], decreasing the stability of the microtubule attachments (as shown by increased phosphorylation of Ndc80). However, little is known about the processes that happen on the sister kinetochore attached to the depolymerizing microtubule. Interesting model could be proposed: as Aurora B kinase activity is increased on the kinetochore attached to the polymerizing microtubule, the activity and/or localization of phosphatases should be increased on the opposite kinetochore. The ability of the Ska complex to track depolymerizing ends of microtubules suggests that it may play a role as a counterpart of EB1 during chromosomal oscillations. It is likely that Ska accumulates PP1 on the depolymerizing microtubule end, gradually overcoming Aurora B kinase activity and allowing for the dephosphorylation of the Ndc80 tail, thus strengthening the microtubule attachments. Interestingly, PP1 is enriched on the opposite sister kinetochore to the one with increased Aurora B activity (Gary Gorbsky, personal communication). This model, however, does not explain how the kinetochores change the directionality of their 
movements. It is possible that a random catastrophe event of the polymerizing microtubule drives the switch. Also the enrichment of Aurora B and PP1 on the opposing sisters could be an incidental result of the oscillations, not a part of their regulatory mechanism. 


\section{References}

[1] M. Paintrand, M. Moudjou, H.H. Delacroix, M. Bornens, Centrosome Organization and Centriole Architecture : Their Sensitivity to Divalent Cations, J. Struct. Biol. 108 (1992) 107-128. doi:10.1016/1047-8477(92)90011-X.

[2] Y. Zheng, M.L. Wong, B. Alberts, T. Mitchison, Nucleation of microtubule assembly by a $\gamma$ tubulin-containing ring complex, Nature. 378 (1995) 578-583. doi:doi:10.1038/378578a0.

[3] M.W. Kirshner, T. Mitchison, Microtubule dynamics, Nature. 324 (1986) 621-621. doi:10.1038/324621a0.

[4] A.L. Manning, D.A. Compton, Structural and regulatory roles of nonmotor spindle proteins, Curr. Opin. Cell Biol. 20 (2008) 101-106. doi:10.1016/j.ceb.2007.11.004.

[5] K.J. Böhm, W. Vater, H. Fenske, E. Unger, Effect of microtubule-associated proteins on the protofilament number of microtubules assembled in vitro, BBA - Gen. Subj. 800 (1984) 119-126. doi:10.1016/0304-4165(84)90049-7.

[6] R. Dallai, B.A. Afzelius, Microtubular diversity in insect spermatozoa: Results obtained with a new fixative, J. Struct. Biol. 103 (1990) 164-179. doi:10.1016/1047-8477(90)90020-D.

[7] D. Chrétien, R.H. Wade, New data on the microtubule surface lattice., Biol. Cell. 71 (1991) 161174. doi:0248-4900(91)90062-R [pii].

[8] R. Dallai, P. Lupetti, C. Mencarelli, Unusual Axonemes of Hexapod Spermatozoa, Int. Rev. Cytol. 254 (2006) 45-99. doi:10.1016/S0074-7696(06)54002-1.

[9] E.E.M.E. Mandelkow, E.E.M.E. Mandelkow, R.A. Milligan, Microtubule dynamics and microtubule caps: A time-resolved cryo-electron microscopy study, J. Cell Biol. 114 (1991) 977991. doi:10.1083/jcb.114.5.977.

[10] T. Mitchison, M. Kirschner, Dynamic instability of microtubule growth., Nature. 312 (1984) 23742. doi:10.1038/312237a0.

[11] A. Desai, T.J. Mitchison, Microtubule polymerization dynamics., Annu. Rev. Cell Dev. Biol. 13 (1997) 83-117. doi:10.1146/annurev.cellbio.13.1.83.

[12] D. Kristofferson, T. Mitchison, M. Kirschner, Direct observation of steady-state microtubule dynamics, J. Cell Biol. 102 (1986) 1007-1019. doi:10.1083/jcb.102.3.1007.

[13] K. Kinoshita, I. Arnal, A. Desai, D.N. Drechsel, A.A. Hyman, Reconstitution of physiological microtubule dynamics using purified components., Science. 294 (2001) 1340-3. doi:10.1126/science.1064629.

[14] S.S.L. Andersen, T. Wittmann, Toward reconstitution of in vivo microtubule dynamics in vitro, BioEssays. 24 (2002) 305-307. doi:10.1002/bies.10084.

[15] Y. Moroi, C. Peebles, M.J. Fritzler, J. Steigerwald, E.M. Tan, Autoantibody to centromere (kinetochore) in scleroderma sera., Proc. Natl. Acad. Sci. U. S. A. 77 (1980) 1627-31. doi:10.1073/pnas.77.3.1627.

[16] W.C. Earnshaw, N. Rothfield, Identification of a family of human centromere proteins using autoimmune sera from patients with scleroderma., Chromosoma. 91 (1985) 313-321. doi:10.1007/BF00328227.

[17] I.M. Cheeseman, A. Desai, Molecular architecture of the kinetochore-microtubule interface., Nat. Rev Mol. Cell Biol. 9 (2008) 33-46. doi:10.1038/nrm2310.

[18] J.P.I. Welburn, I.M. Cheeseman, Toward a Molecular Structure of the Eukaryotic Kinetochore, Dev. Cell. 15 (2008) 645-655. doi:10.1016/j.devcel.2008.10.011.

[19] E. a Foley, T.M. Kapoor, Microtubule attachment and spindle assembly checkpoint signalling at the kinetochore., Nat. Rev. Mol. Cell Biol. 14 (2013) 25-37. doi:10.1038/nrm3494.

[20] I.M. Cheeseman, The kinetochore., Int. Rev. Cytol. 94 (2014) 77-105. doi:10.1101/cshperspect.a015826. 
[21] B.R. Brinkley, E. Stubblefield, The fine structure of the kinetochore of a mammalian cell in vitro, Chromosoma. 19 (1966) 28-43. doi:10.1007/BF00332792.

[22] P.T. Jokelainen, The ultrastructure and spatial organization of the metaphase kinetochore in mitotic rat cells, J. Ultrastruct. Res. 19 (1967) 19-44. doi:10.1016/S0022-5320(67)80058-3.

[23] D.E. Comings, T.A. Okada, Fine structure of kinetochore in Indian muntjac, Exp. Cell Res. 67 (1971) 97-110. doi:10.1016/0014-4827(71)90625-2.

[24] U.P. Roos, Light and electron microscopy of rat kangaroo cells in mitosis. III. Patterns of chromosome behavior during prometaphase., Chromosoma. 54 (1976) 363-385. doi:10.1007/BF00319696.

[25] B.F. McEwen, Y. Ding, A.B. Heagle, Relevance of kinetochore size and microtubule-binding capacity for stable chromosome attachment during mitosis in PtK1 cells, Chromosom. Res. 6 (1998) 123-132. doi:10.1023/A:1009239013215.

[26] T.J. Mitchison, M.W. Kirschner, Properties of the kinetochore in vitro. I. Microtubule nucleation and tubulin binding, J. Cell Biol. 101 (1985) 755-765. doi:10.1083/jcb.101.3.755.

[27] B.F. McEwen, Y. Dong, K.J. VandenBeldt, Using Electron Microscopy to Understand Functional Mechanisms of Chromosome Alignment on the Mitotic Spindle, Methods Cell Biol. 2007 (2007) 259-293. doi:10.1016/S0091-679X(06)79011-2.

[28] a F. Pluta, a M. Mackay, a M. Ainsztein, I.G. Goldberg, W.C. Earnshaw, The centromere: hub of chromosomal activities., Science. 270 (1995) 1591-1594. doi:10.1126/science.270.5242.1591.

[29] S. Pimpinelli, C. Goday, Unusual kinetochores and chromatin diminution in Parascaris, Trends Genet. 5 (1989) 310-315. doi:10.1016/0168-9525(89)90114-5.

[30] B.F. McEwen, G.K. Chan, B. Zubrowski, M.S. Savoian, M.T. Sauer, T.J. Yen, CENP-E is essential for reliable bioriented spindle attachment, but chromosome alignment can be achieved via redundant mechanisms in mammalian cells., Mol. Biol. Cell. 12 (2001) 2776-2789. doi:10.1091/mbc.12.9.2776.

[31] F.A. Steiner, S. Henikoff, Holocentromeres are dispersed point centromeres localized at transcription factor hotspots, Elife. 2014 (2014). doi:10.7554/eLife.02025.

[32] D.K. Palmer, K. O’Day, M.H. Wener, B.S. Andrews, R.L. Margolis, A 17-kD centromere protein (CENP-A) copurifies with nucleosome core particles and with histones, J. Cell Biol. 104 (1987) 805-815. doi:10.1083/jcb.104.4.805.

[33] D.K. Palmer, K. O’Day, R.L. Margolis, Biochemical analysis of CENP-A, a centromeric protein with histone-like properties, Prog. Clin. Biol. Res. 318 (1989) 61-72.

[34] D.K. Palmer, H. LE Trong, H. Charbonneau, R.L. Margolis, Purification of the centromere-specific protein CENP-A and demonstration that it is a distinctive histone, Cell Biol. 88 (1991) 3734-3738.

[35] S. Stoler, K.C. Keith, K.F.E. Kurnick, M. Fitzgerald-Hayes, A mutation in CSE4, an essential gene encoding a novel chromatin- associated protein in yeast, causes chromosome nondisjunction and cell cycle arrest at mitosis, Genes Dev. 9 (1995) 573-586. doi:10.1101/gad.9.5.573.

[36] S.-T.T. Liu, J.B. Rattner, S.A. Jablonski, T.J. Yen, Mapping the assembly pathways that specify formation of the trilaminar kinetochore plates in human cells, J. Cell Biol. 175 (2006) 41-53. doi:10.1083/jcb.200606020.

[37] C.W. Carroll, K.J. Milks, A.F. Straight, Dual recognition of CENP-A nucleosomes is required for centromere assembly, J. Cell Biol. 189 (2010) 1143-1155. doi:10.1083/jcb.201001013.

[38] M. Perpelescu, T. Fukagawa, The ABCs of CENPs, Chromosoma. 120 (2011) 425-446. doi:10.1007/s00412-011-0330-0.

[39] K.E. Gascoigne, K. Takeuchi, A. Suzuki, T. Hori, T. Fukagawa, I.M. Cheeseman, Induced ectopic kinetochore assembly bypasses the requirement for CENP-A nucleosomes, Cell. 145 (2011) 410422. doi:10.1016/j.cell.2011.03.031.

[40] M.R. Przewloka, Z. Venkei, V.M. Bolanos-Garcia, J. Debski, M. Dadlez, D.M. Glover, CENP-C is a structural platform for kinetochore assembly, Curr. Biol. 21 (2011) 399-405. doi:10.1016/j.cub.2011.02.005. 
[41] E. Screpanti, A. De Antoni, G.M. Alushin, A. Petrovic, T. Melis, E. Nogales, A. Musacchio, Direct binding of Cenp-C to the Mis 12 complex joins the inner and outer kinetochore, Curr. Biol. 21 (2011) 391-398. doi:10.1016/j.cub.2010.12.039.

[42] I.M. Cheeseman, J.S. Chappie, E.M. Wilson-Kubalek, A. Desai, The Conserved KMN Network Constitutes the Core Microtubule-Binding Site of the Kinetochore, Cell. 127 (2006) 983-997. doi:10.1016/j.cell.2006.09.039.

[43] M. Emanuele, M. McCleland, D. Satinover, P. Stukenberg, Measuring the stoichiometry and physical interactions between components elucidates the architecture of the vertebrate kinetochore, Mol. Biol. Cell. 16 (2005) 4882-4892. doi:10.1091/mbc.E05-03-0239.

[44] A. Petrovic, S. Pasqualato, P. Dube, V. Krenn, S. Santaguida, D. Cittaro, S. Monzani, L. Massimiliano, J. Keller, A. Tarricone, A. Maiolica, H. Stark, A. Musacchio, The MIS12 complex is a protein interaction hub for outer kinetochore assembly, J. Cell Biol. 190 (2010) 835-852. doi:10.1083/jcb.201002070.

[45] T. Kiyomitsu, H. Murakami, M. Yanagida, Protein Interaction Domain Mapping of Human Kinetochore Protein Blinkin Reveals a Consensus Motif for Binding of Spindle Assembly Checkpoint Proteins Bub1 and BubR1, Mol. Cell. Biol. 31 (2011) 998-1011. doi:10.1128/MCB.00815-10.

[46] B.J.J. Howell, B.F.F. McEwen, J.C.C. Canman, D.B.B. Hoffman, E.M.M. Farrar, C.L.L. Rieder, E.D.D. Salmon, Cytoplasmic dynein/dynactin drives kinetochore protein transport to the spindle poles and has a role in mitotic spindle checkpoint inactivation, J. Cell Biol. 155 (2001) 1159-1172. doi:10.1083/jcb.200105093.

[47] T.M. Kapoor, M. a Lampson, P. Hergert, L. Cameron, D. Cimini, E.D. Salmon, B.F. McEwen, A. Khodjakov, Chromosomes Can Congress to the Metaphase Plate Before Biorientation, Science. 311 (2006) 388-391. doi:10.1126/science.1122142.

[48] V. V. Vorozhko, M.J. Emanuele, M.J. Kallio, P.T. Stukenberg, G.J. Gorbsky, Multiple mechanisms of chromosome movement in vertebrate cells mediated through the Ndc80 complex and dynein/dynactin, Chromosoma. 117 (2008) 169-179. doi:10.1007/s00412-007-0135-3.

[49] D.K. Cheerambathur, A. Desai, Linked in: Formation and regulation of microtubule attachments during chromosome segregation, Curr. Opin. Cell Biol. 26 (2014) 113-122. doi:10.1016/j.ceb.2013.12.005.

[50] L. Clarke, J. Carbon, The structure and function of yeast centromeres, Annu. Rev. Genet. 19 (1985) 29-56. doi:10.1002/yea.

[51] M. Fitzgerald-Hayes, L. Clarke, J. Carbon, Nucleotide sequence comparisons and functional analysis of yeast centromere DNAs, Cell. 29 (1982) 235-244. doi:10.1016/0092-8674(82)90108-8.

[52] K.S. Bloom, J. Carbon, Yeast centromere DNA is in a unique and highly ordered structure in chromosomes and small circular minichromosomes, Cell. 29 (1982) 305-317. doi:10.1016/00928674(82)90147-7.

[53] D.W. Cleveland, Y. Mao, K.F. Sullivan, Centromeres and kinetochores: From epigenetics to mitotic checkpoint signaling, Cell. 112 (2003) 407-421. doi:10.1016/S0092-8674(03)00115-6.

[54] B.E. Black, D.W. Cleveland, Epigenetic centromere propagation and the nature of CENP-A nucleosomes, Cell. 144 (2011) 471-479. doi:10.1016/j.cell.2011.02.002.

[55] H. Kato, J. Jiang, B.-R. Zhou, M. Rozendaal, H. Feng, R. Ghirlando, T.S. Xiao, A.F. Straight, Y. $\mathrm{Bai}, \mathrm{A}$ conserved mechanism for centromeric nucleosome recognition by centromere protein CENP-C., Science. 340 (2013) 1110-3. doi:10.1126/science.1235532.

[56] S.-T. Liu, J.C. Hittle, S.A. Jablonski, M.S. Campbell, K. Yoda, T.J. Yen, Human CENP-I specifies localization of CENP-F, MAD1 and MAD2 to kinetochores and is essential for mitosis., Nat. Cell Biol. 5 (2003) 341-5. doi:10.1038/ncb953.

[57] M.-S. Kwon, T. Hori, M. Okada, T. Fukagawa, CENP-C is involved in chromosome segregation, mitotic checkpoint function, and kinetochore assembly., Mol. Biol. Cell. 18 (2007) 2155-68. doi:10.1091/mbc.E07-01-0045.

[58] K.J. Milks, B. Moree, A.F. Straight, Dissection of CENP-C-directed centromere and kinetochore assembly., Mol. Biol. Cell. 20 (2009) 4246-4255. doi:10.1091/mbc.E09-05-0378. 
[59] A. Musacchio, E.D. Salmon, The spindle-assembly checkpoint in space and time., Nat. Rev. Mol. Cell Biol. 8 (2007) 379-93. doi:10.1038/nrm2163.

[60] T. Nishino, K. Takeuchi, K.E. Gascoigne, A. Suzuki, T. Hori, T. Oyama, K. Morikawa, I.M. Cheeseman, T. Fukagawa, CENP-T-W-S-X forms a unique centromeric chromatin structure with a histone-like fold, Cell. 148 (2012) 487-501. doi:10.1016/j.cell.2011.11.061.

[61] A. Schleiffer, M. Maier, G. Litos, F. Lampert, P. Hornung, K. Mechtler, S. Westermann, CENP-T proteins are conserved centromere receptors of the Ndc80 complex, Nat. Cell Biol. 14 (2012) 1-12. doi:10.1038/ncb2493.

[62] T. Hori, W.H. Shang, K. Takeuchi, T. Fukagawa, The CCAN recruits CENP-A to the centromere and forms the structural core for kinetochore assembly, J. Cell Biol. 200 (2013) 45-60. doi:10.1083/jcb.201210106.

[63] A.P. Joglekar, D.C. Bouck, J.N. Molk, K.S. Bloom, E.D. Salmon, Molecular architecture of a kinetochore-microtubule attachment site, Nat. Cell Biol. 8 (2006) 581-585. doi:10.1038/ncb1414.

[64] A.P. Joglekar, E.D. Salmon, K.S. Bloom, Counting Kinetochore Protein Numbers in Budding Yeast Using Genetically Encoded Fluorescent Proteins, Methods Cell Biol. 85 (2008) 127-151. doi:10.1016/S0091-679X(08)85007-8.

[65] K. Johnston, A. Joglekar, T. Hori, A. Suzuki, T. Fukagawa, E.D. Salmon, Vertebrate kinetochore protein architecture: Protein copy number, J. Cell Biol. 189 (2010) 937-943. doi:10.1083/jcb.200912022.

[66] X. Liu, I. McLeod, S. Anderson, J.R. Yates, X. He, Molecular analysis of kinetochore architecture in fission yeast., EMBO J. 24 (2005) 2919-2930. doi:10.1038/sj.emboj.7600762.

[67] I. Sanchez-Perez, S.J. Renwick, K. Crawley, I. Karig, V. Buck, J.C. Meadows, A. Franco-Sanchez, U. Fleig, T. Toda, J.B.A. Millar, The DASH complex and Klp5/Klp6 kinesin coordinate bipolar chromosome attachment in fission yeast., EMBO J. 24 (2005) 2931-2943. doi:10.1038/sj.emboj.7600761.

[68] V. Magidson, J. He, J.G. Ault, C.B.O. Connell, N. Yang, I. Tikhonenko, B.F. Mcewen, H. Sui, A. Khodjakov, Unattached kinetochores rather than intrakinetochore tension arrest mitosis in taxoltreated cells, J. Cell Biol. 212 (2016) 307-319. doi:10.1083/jcb.201412139.

[69] A. V. Zaytsev, L.J.R. Sundin, K.F. DeLuca, E.L. Grishchuk, J.G. DeLuca, Accurate phosphoregulation of kinetochore-microtubule affinity requires unconstrained molecular interactions, J. Cell Biol. 206 (2014) 45-59. doi:10.1083/jcb.201312107.

[70] V. Magidson, R. Paul, N. Yang, J.G. Ault, C.B. O'Connell, I. Tikhonenko, B.F. McEwen, A. Mogilner, A. Khodjakov, Adaptive changes in the kinetochore architecture facilitate proper spindle assembly., Nat. Cell Biol. 17 (2015) 1134-44. doi:10.1038/ncb3223.

[71] J. Richard McIntosh, E. O’Toole, K. Zhudenkov, M. Morphew, C. Schwartz, F.I. Ataullakhanov, E.L. Grishchuk, Conserved and divergent features of kinetochores and spindle microtubule ends from five species, J. Cell Biol. 200 (2013) 459-474. doi:10.1083/jcb.201209154.

[72] Y. Dong, K.J. Vanden Beldt, X. Meng, A. Khodjakov, B.F. McEwen, The outer plate in vertebrate kinetochores is a flexible network with multiple microtubule interactions., Nat. Cell Biol. 9 (2007) 516-22. doi:10.1038/ncb1576.

[73] B. Etemad, G.J. Kops, Attachment issues: kinetochore transformations and spindle checkpoint silencing, Curr. Opin. Cell Biol. 39 (2016) 101-108. doi:10.1016/j.ceb.2016.02.016.

[74] C.M. Pfarr, M. Coue, P.M. Grissom, T.S. Hays, M.E. Porter, J.R. McIntosh, Cytoplasmic dynein is localized to kinetochores during mitosis., Nature. 345 (1990) 263-265. doi:10.1038/345263a0.

[75] T.J. Yen, G. Li, B.T. Schaar, I. Szilak, D.W. Cleveland, CENP-E is a putative kinetochore motor that accumulates just before mitosis., Nature. 359 (1992) 536-539. doi:10.1038/359536a0.

[76] T. Shima, T. Kon, K. Imamula, R. Ohkura, K. Sutoh, Two modes of microtubule sliding driven by cytoplasmic dynein., Proc. Natl. Acad. Sci. U. S. A. 103 (2006) 17736-17740. doi:10.1073/pnas.0606794103.

[77] S.S. Rosenfeld, M. van Duffelen, W.M. Behnke-Parks, C. Beadle, J. Correia, J. Xing, A ATPase cycle of the mitotic motor CENP-E, J. Biol. Chem. 284 (2009) 32858-32868. 


\section{doi:10.1074/jbc.M109.041210.}

[78] S. Cai, C.B. O'Connell, A. Khodjakov, C.E. Walczak, Chromosome congression in the absence of kinetochore fibres., Nat. Cell Biol. 11 (2009) 832-838. doi:10.1038/ncb1890.

[79] V. Magidson, C.B. O’Connell, J. Lončarek, R. Paul, A. Mogilner, A. Khodjakov, The spatial arrangement of chromosomes during prometaphase facilitates spindle assembly, Cell. 146 (2011) 555-567. doi:10.1016/j.cell.2011.07.012.

[80] R.L. Shrestha, V.M. Draviam, Lateral to end-on conversion of chromosome-microtubule attachment requires kinesins CENP-E and MCAK, Curr. Biol. 23 (2013) 1514-1526. doi:10.1016/j.cub.2013.06.040.

[81] F. Rago, I.M. Cheeseman, The functions and consequences of force at kinetochores, J. Cell Biol. 200 (2013) 557-565. doi:10.1083/jcb.201211113.

[82] K. Tanaka, N. Mukae, H. Dewar, M. van Breugel, E.K. James, A.R. Prescott, C. Antony, T.U. Tanaka, Molecular mechanisms of kinetochore capture by spindle microtubules., Nature. 434 (2005) 987-994. doi:10.1038/nature03483.

[83] P.A. Wigge, J. V Kilmartin, The Ndc80p complex from Saccharomyces cerevisiae contains conserved centromere components and has a function in chromosome segregation, J. Cell Biol. 152 (2001) 349-360. doi:10.1083/jcb.152.2.349.

[84] J.G. DeLuca, B. Moree, J.M. Hickey, J. V. Kilmartin, E.D. Salmon, hNuf2 inhibition blocks stable kinetochore-microtubule attachment and induces mitotic cell death in HeLa cells., J. Cell Biol. 159 (2002) 549-555. doi:10.1083/jcb.200208159.

[85] S. Martin-Lluesma, V.M. Stucke, E. a Nigg, Role of Hec1 in spindle checkpoint signaling and kinetochore recruitment of Mad1/Mad2., Science. 297 (2002) 2267-2270. doi:10.1126/science.1075596.

[86] M.L. McCleland, R.D. Gardner, M.J. Kallio, J.R. Daum, G.J. Gorbsky, D.J. Burke, P.T. Stukenberg, The highly conserved Ndc80 complex is required for kinetochore assembly, chromosome congression, and spindle checkpoint activity, Genes Dev. 17 (2003) 101-114. doi:10.1101/gad.1040903.

[87] M.L. McCleland, M.J. Kallio, G.A. Barrett-Wilt, C.A. Kestner, J. Shabanowitz, D.F. Hunt, G.J. Gorbsky, P.T. Stukenberg, The Vertebrate Ndc80 Complex Contains Spc24 and Spc25 Homologs, which Are Required to Establish and Maintain Kinetochore-Microtubule Attachment, Curr. Biol. 14 (2004) 131-137. doi:10.1016/j.cub.2003.12.058.

[88] D. Varma, E.D. Salmon, The KMN protein network - chief conductors of the kinetochore orchestra, J. Cell Sci. 125 (2012) 5927-5936. doi:10.1242/jcs.093724.

[89] H. Funabiki, D.J. Wynne, Making an effective switch at the kinetochore by phosphorylation and dephosphorylation, Chromosoma. 122 (2013) 135-158.

[90] C. Ciferri, J. De Lucall, S. Monzani, K.J. Ferrari, D. Ristic, C. Wyman, H. Stark, J. Kilmartin, E.D. Salmon, A. Musacchio, Architecture of the human Ndc80-Hec1 complex, a critical constituent of the outer kinetochore, J. Biol. Chem. 280 (2005) 29088-29095. doi:10.1074/jbc.M504070200.

[91] R.R. Wei, P.K. Sorger, S.C. Harrison, Molecular organization of the Ndc80 complex, an essential kinetochore component., Proc. Natl. Acad. Sci. U. S. A. 102 (2005) 5363-7. doi:10.1073/pnas.0501168102.

[92] H.W. Wang, S. Long, C. Ciferri, S. Westermann, D. Drubin, G. Barnes, E. Nogales, Architecture and Flexibility of the Yeast Ndc80 Kinetochore Complex, J. Mol. Biol. 383 (2008) 894-903. doi:10.1016/j.jmb.2008.08.077.

[93] C. Ciferri, S. Pasqualato, E. Screpanti, G. Varetti, S. Santaguida, G. Dos Reis, A. Maiolica, J. Polka, J.G. De Luca, P. De Wulf, M. Salek, J. Rappsilber, C.A. Moores, E.D. Salmon, A. Musacchio, Implications for Kinetochore-Microtubule Attachment from the Structure of an Engineered Ndc80 Complex, Cell. 133 (2008) 427-439. doi:10.1016/j.cell.2008.03.020.

[94] R.R. Wei, J.R. Schnell, N.A. Larsen, P.K. Sorger, J.J. Chou, S.C. Harrison, Structure of a Central Component of the Yeast Kinetochore: The Spc24p/Spc25p Globular Domain, Structure. 14 (2006) 1003-1009. doi:10.1016/j.str.2006.04.007. 
[95] A. Suzuki, B.L. Badger, E.D. Salmon, A quantitative description of Ndc80 complex linkage to human kinetochores., Nat. Commun. 6 (2015) 8161. doi:10.1038/ncomms9161.

[96] F. Malvezzi, G. Litos, A. Schleiffer, A. Heuck, K. Mechtler, T. Clausen, S. Westermann, A structural basis for kinetochore recruitment of the Ndc80 complex via two distinct centromere receptors., EMBO J. 32 (2013) 409-23. doi:10.1038/emboj.2012.356.

[97] R.R. Wei, J. Al-Bassam, S.C. Harrison, The Ndc80/HEC1 complex is a contact point for kinetochore-microtubule attachment., Nat. Struct. Mol. Biol. 14 (2007) 54-59. doi:10.1038/nsmb1186.

[98] E.M. Kudalkar, E.A. Scarborough, N.T. Umbreit, A. Zelter, D.R. Gestaut, M. Riffle, R.S. Johnson, M.J. MacCoss, C.L. Asbury, T.N. Davis, Regulation of outer kinetochore Ndc80 complex-based microtubule attachments by the central kinetochore Mis12/MIND complex, Proc. Natl. Acad. Sci. U. S. A. 112 (2015) E5583-9. doi:10.1073/pnas.1513882112.

[99] E.M. Wilson-Kubalek, I.M. Cheeseman, C. Yoshioka, A. Desai, R.A. Milligan, Orientation and structure of the Ndc80 complex on the microtubule lattice, J. Cell Biol. 182 (2008) 1055-1061. doi:10.1083/jcb.200804170.

[100] K.S. Hsu, T. Toda, Ndc80 internal loop interacts with Dis1/TOG to ensure proper kinetochorespindle attachment in fission yeast, Curr. Biol. 21 (2011) 214-220. doi:10.1016/j.cub.2010.12.048.

[101] J.F. Maure, S. Komoto, Y. Oku, A. Mino, S. Pasqualato, K. Natsume, L. Clayton, A. Musacchio, T.U. Tanaka, The Ndc80 loop region facilitates formation of kinetochore attachment to the dynamic microtubule plus end, Curr. Biol. 21 (2011) 207-213. doi:10.1016/j.cub.2010.12.050.

[102] D. Varma, S. Chandrasekaran, L.J.R. Sundin, K.T. Reidy, X. Wan, D.A.D. Chasse, K.R. Nevis, J.G. DeLuca, E.D. Salmon, J.G. Cook, Recruitment of the human Cdt1 replication licensing protein by the loop domain of $\mathrm{Hec} 1$ is required for stable kinetochore-microtubule attachment., Nat. Cell Biol. 14 (2012) 593-603. doi:10.1038/ncb2489.

[103] G. Zhang, C.D. Kelstrup, X.-W. Hu, M.J. Kaas Hansen, M.R. Singleton, J. V Olsen, J. Nilsson, The Ndc80 internal loop is required for recruitment of the Ska complex to establish end-on microtubule attachment to kinetochores, J. Cell Sci. 125 (2012) 3243-3253. doi:10.1242/jcs.104208.

[104] G.J. Guimaraes, Y. Dong, B.F. McEwen, J.G. DeLuca, Kinetochore-Microtubule Attachment Relies on the Disordered N-Terminal Tail Domain of Hec1, Curr. Biol. 18 (2008) 1778-1784. doi:10.1016/j.cub.2008.08.012.

[105] S.A. Miller, M.L. Johnson, P.T. Stukenberg, Kinetochore Attachments Require an Interaction between Unstructured Tails on Microtubules and Ndc80Hec1, Curr. Biol. 18 (2008) 1785-1791. doi:10.1016/j.cub.2008.11.007.

[106] S. Kemmler, M. Stach, M. Knapp, J. Ortiz, J. Pfannstiel, T. Ruppert, J. Lechner, Mimicking Ndc80 phosphorylation triggers spindle assembly checkpoint signalling., EMBO J. 28 (2009) 1099-1110. doi:10.1038/emboj.2009.62.

[107] J.G. Tooley, S.A. Miller, P.T. Stukenberg, The Ndc80 complex uses a tripartite attachment point to couple microtubule depolymerization to chromosome movement., Mol. Biol. Cell. 22 (2011) 12171226. doi:10.1091/mbc.E10-07-0626.

[108] G.M. Alushin, V.H. Ramey, S. Pasqualato, D.A. Ball, N. Grigorieff, A. Musacchio, E. Nogales, The Ndc80 kinetochore complex forms oligomeric arrays along microtubules., Nature. 467 (2010) 805-10. doi:10.1038/nature09423.

[109] E.M. Wilson-Kubalek, I.M. Cheeseman, R.A. Milligan, Structural comparison of the C. elegans and human Ndc80 complexes bound to microtubules reveals distinct binding behavior., Mol. Biol. Cell. (2016). doi:10.1016/j.plasmid.2003.10.003.

[110] J.C. Schmidt, H. Arthanari, A. Boeszoermenyi, N.M. Dashkevich, E.M. Wilson-Kubalek, N. Monnier, M. Markus, M. Oberer, R.A. Milligan, M. Bathe, G. Wagner, E.L. Grishchuk, I.M. Cheeseman, The Kinetochore-Bound Ska1 Complex Tracks Depolymerizing Microtubules and Binds to Curved Protofilaments, Dev. Cell. 23 (2012) 968-980. doi:10.1016/j.devcel.2012.09.012.

[111] M. Nousiainen, H.H.W. Silljé, G. Sauer, E.A. Nigg, R. Körner, Phosphoproteome analysis of the human mitotic spindle., Proc. Natl. Acad. Sci. U. S. A. 103 (2006) 5391-5396. doi:10.1073/pnas.0507066103. 
[112] J.G. DeLuca, W.E. Gall, C. Ciferri, D. Cimini, A. Musacchio, E.D. Salmon, Kinetochore Microtubule Dynamics and Attachment Stability Are Regulated by Hec1, Cell. 127 (2006) 969982. doi:10.1016/j.cell.2006.09.047.

[113] R. Malik, R. Lenobel, A. Santamaria, A. Ries, E.A. Nigg, R. Körner, Quantitative analysis of the human spindle phosphoproteome at distinct mitotic stages, J. Proteome Res. 8 (2009) 4553-4563. doi:10.1021/pr9003773.

[114] S. Santaguida, A. Musacchio, The life and miracles of kinetochores., EMBO J. 28 (2009) 2511-31. doi:10.1038/emboj.2009.173.

[115] K.F. DeLuca, S.M.A. Lens, J.G. DeLuca, Temporal changes in Hec1 phosphorylation control kinetochore-microtubule attachment stability during mitosis, J. Cell Sci. 124 (2011) 622-634. doi:10.1242/jcs.072629.

[116] N.T. Umbreit, D.R. Gestaut, J.F. Tien, B.S. Vollmar, T. Gonen, C.L. Asbury, T.N. Davis, The Ndc80 kinetochore complex directly modulates microtubule dynamics., Proc. Natl. Acad. Sci. U. S. A. 109 (2012) 16113-8. doi:10.1073/pnas.1209615109.

[117] G.M. Alushin, V. Musinipally, D. Matson, J. Tooley, P.T. Stukenberg, E. Nogales, Multimodal microtubule binding by the Ndc80 kinetochore complex., Nat. Struct. Mol. Biol. 19 (2012) 1161-7. doi:10.1038/nsmb.2411.

[118] A.F. Powers, A.D. Franck, D.R. Gestaut, J. Cooper, B. Gracyzk, R.R. Wei, L. Wordeman, T.N. Davis, C.L. Asbury, The Ndc80 Kinetochore Complex Forms Load-Bearing Attachments to Dynamic Microtubule Tips via Biased Diffusion, Cell. 136 (2009) 865-875. doi:10.1016/j.cell.2008.12.045.

[119] J.F. Tien, N.T. Umbreit, D.R. Gestaut, A.D. Franck, J. Cooper, L. Wordeman, T. Gonen, C.L. Asbury, T.N. Davis, Cooperation of the Dam1 and Ndc80 kinetochore complexes enhances microtubule coupling and is regulated by aurora B, J. Cell Biol. 189 (2010) 713-723. doi:10.1083/jcb.200910142.

[120] A. V Zaytsev, J.E. Mick, E. Maslennikov, B. Nikashin, J.G. Deluca, Multisite phosphorylation of the NDC80 complex gradually tunes its microtubule-binding affinity, Mol. Biol. Cell. 26 (2015) 1829-1844. doi:10.1091/mbc.E14-11-1539.

[121] J.P.I. Welburn, E.L. Grishchuk, C.B. Backer, E.M. Wilson-Kubalek, J.R. Yates, I.M. Cheeseman, The Human Kinetochore Ska1 Complex Facilitates Microtubule Depolymerization-Coupled Motility, Dev. Cell. 16 (2009) 374-385. doi:10.1016/j.devcel.2009.01.011.

[122] J. Lawrimore, K.S. Bloom, E.D. Salmon, Point centromeres contain more than a single centromerespecific Cse4 (CENP-A) nucleosome, J. Cell Biol. 195 (2011) 573-582. doi:10.1083/jcb.201106036.

[123] I.M. Cheeseman, M. Enquist-Newman, T. M??ller-Reichert, D.G. Drubin, G. Barnes, Mitotic spindle integrity and kinetochore function linked by the Duo1p/Dam1p complex, J. Cell Biol. 152 (2001) 197-212. doi:10.1083/jcb.152.1.197.

[124] C. Hofmann, I.M. Cheeseman, B.L. Goode, K.L. McDonald, G. Barnes, D.G. Drubin, Saccharomyces cerevisiae Duo1p and Dam1p, Novel Proteins Involved in Mitotic Spindle Function, J. Cell Biol. 143 (1998) 1029-1040. doi:10.1083/jcb.143.4.1029.

[125] M.H. Jones, J.B. Bachant, A.R. Castillo, T.H. Giddings, M. Winey, Yeast Dam1p is required to maintain spindle integrity during mitosis and interacts with the Mps1p kinase., Mol. Biol. Cell. 10 (1999) 2377-91. doi:10.1091/mbc.10.7.2377.

[126] C. Janke, J. Ortiz, T.U. Tanaka, J. Lechner, E. Schiebel, Four new subunits of the Dam1-Duo1 complex reveal novel functions in sister kinetochore biorientation, EMBO J. 21 (2002) 181-193. doi:10.1093/emboj/21.1.181.

[127] M.H. Jones, X. He, T.H. Giddings, M. Winey, Yeast Dam1p has a role at the kinetochore in assembly of the mitotic spindle., Proc. Natl. Acad. Sci. U. S. A. 98 (2001) 13675-80. doi:10.1073/pnas.241417098.

[128] S. Westermann, H.-W. Wang, A. Avila-Sakar, D.G. Drubin, E. Nogales, G. Barnes, The Dam1 kinetochore ring complex moves processively on depolymerizing microtubule ends., Nature. 440 (2006) 565-569. doi:10.1038/nature04409. 
[129] J.J.L. Miranda, P. De Wulf, P.K. Sorger, S.C. Harrison, The yeast DASH complex forms closed rings on microtubules., Nat. Struct. Mol. Biol. 12 (2005) 138-43. doi:10.1038/nsmb896.

[130] D.R. Gestaut, B. Graczyk, J. Cooper, P.O. Widlund, A. Zelter, L. Wordeman, C.L. Asbury, T.N. Davis, Phosphoregulation and depolymerization-driven movement of the Dam1 complex do not require ring formation., Nat. Cell Biol. 10 (2008) 407-414. doi:10.1038/ncb1702.

[131] F. Lampert, P. Hornung, S. Westermann, The Dam1 complex confers microtubule plus endtracking activity to the Ndc80 kinetochore complex, J. Cell Biol. 189 (2010) 641-649. doi:10.1083/jcb.200912021.

[132] G. Sauer, R. Körner, A. Hanisch, A. Ries, E.A. Nigg, H.H.W. Silljé, Proteome analysis of the human mitotic spindle., Mol. Cell. Proteomics. 4 (2005) 35-43. doi:10.1074/mcp.M400158MCP200.

[133] A. Hanisch, H.H.W. Silljé, E.A. Nigg, Timely anaphase onset requires a novel spindle and kinetochore complex comprising Ska1 and Ska2., EMBO J. 25 (2006) 5504-5515. doi:10.1038/sj.emboj.7601426.

[134] M. Theis, M. Slabicki, M. Junqueira, M. Paszkowski-Rogacz, J. Sontheimer, R. Kittler, A.-K. Heninger, T. Glatter, K. Kruusmaa, I. Poser, A.A. Hyman, M.T. Pisabarro, M. Gstaiger, R. Aebersold, A. Shevchenko, F. Buchholz, Comparative profiling identifies C13orf3 as a component of the Ska complex required for mammalian cell division., EMBO J. 28 (2009) 1453-1465. doi:10.1038/emboj.2009.114.

[135] T.N. Gaitanos, A. Santamaria, a A. Jeyaprakash, B. Wang, E. Conti, E.A. Nigg, Stable kinetochore-microtubule interactions depend on the Ska complex and its new component Ska3/C13Orf3., EMBO J. 28 (2009) 1442-1452. doi:10.1038/emboj.2009.96.

[136] J.A. Raaijmakers, M.E. Tanenbaum, A.F. Maia, R.H. Medema, RAMA1 is a novel kinetochore protein involved in kinetochore-microtubule attachment., J. Cell Sci. 122 (2009) 2436-2445. doi:10.1242/jcs.051912.

[137] J.R. Daum, J.D. Wren, J.J. Daniel, S. Sivakumar, J.N. McAvoy, T.A. Potapova, G.J. Gorbsky, Ska3 Is Required for Spindle Checkpoint Silencing and the Maintenance of Chromosome Cohesion in Mitosis, Curr. Biol. 19 (2009) 1467-1472. doi:10.1016/j.cub.2009.07.017.

[138] S. Sivakumar, J.R. Daum, A.R. Tipton, S. Rankin, G.J. Gorbsky, The spindle and kinetochoreassociated (Ska) complex enhances binding of the anaphase-promoting complex/cyclosome (APC/C) to chromosomes and promotes mitotic exit., Mol. Biol. Cell. 25 (2014) 594-605. doi:10.1091/mbc.E13-07-0421.

[139] Y.W. Chan, A.A. Jeyaprakash, E.A. Nigg, A. Santamaria, Aurora B controls kinetochoremicrotubule attachments by inhibiting Ska complex-KMN network interaction, J. Cell Biol. 196 (2012) 563-571. doi:10.1083/jcb.201109001.

[140] J.R. Daum, T.A. Potapova, S. Sivakumar, J.J. Daniel, J.N. Flynn, S. Rankin, G.J. Gorbsky, Cohesion fatigue induces chromatid separation in cells delayed at metaphase, Curr. Biol. 21 (2011) 1018-1024. doi:10.1016/j.cub.2011.05.032.

[141] S. Ohta, J.C. Bukowski-Wills, L. Sanchez-Pulido, F. de L. Alves, L. Wood, Z.A. Chen, M. Platani, L. Fischer, D.F. Hudson, C.P. Ponting, T. Fukagawa, W.C. Earnshaw, J. Rappsilber, The Protein Composition of Mitotic Chromosomes Determined Using Multiclassifier Combinatorial Proteomics, Cell. 142 (2010) 810-821. doi:10.1016/j.cell.2010.07.047.

[142] Y. Hiruma, C. Sacristan, S.T. Pachis, A. Adamopoulos, T. Kuijt, M. Ubbink, E. von Castelmur, A. Perrakis, G.J. Kops, Competition between MPS1 and microtubules at kinetochores regulates spindle checkpoint signaling, Science (80-. ). 348 (2015) 1264-1267. doi:10.1126/science.aaa4055.

[143] Z. Ji, H. Gao, H. Yu, Kinetochore attachment sensed by competitive Mps1 and microtubule binding to Ndc80C, Science (80-. ). 348 (2015) 1260-1264. doi:10.1126/science.aaa4029.

[144] A.A. Jeyaprakash, A. Santamaria, U. Jayachandran, Y.W. Chan, C. Benda, E.A. Nigg, E. Conti, Structural and Functional Organization of the Ska Complex, a Key Component of the KinetochoreMicrotubule Interface, Mol. Cell. 46 (2012) 274-286. doi:10.1016/j.molcel.2012.03.005.

[145] M.A. Abad, B. Medina, A. Santamaria, J. Zou, C. Plasberg-Hill, A. Madhumalar, U. Jayachandran, P.M. Redli, J. Rappsilber, E.A. Nigg, A.A. Jeyaprakash, Structural basis for microtubule 
recognition by the human kinetochore Ska complex., Nat. Commun. 5 (2014) 2964. doi:10.1038/ncomms3964.

[146] B.R. Brinkley, J. Cartwright, Cold-Labile and Cold-Stable Microtubules in the Mitotic Spindle of Mammalian Cells, Ann. N. Y. Acad. Sci. 253 (1975) 428-439. doi:10.1111/j.17496632.1975.tb19218.x.

[147] C.L. Rieder, The structure of the cold-stable kinetochore fiber in metaphase PtK1 cells, Chromosoma. 84 (1981) 145-158. doi:10.1007/BF00293368.

[148] G.J. Holland, J. a Curry, R.K. Didham, J. Bascompte, S.R. Carpenter, a R. Ives, T.K. Kratz, L. Species, C.G. Jones, J.H. Lawton, C. Pedersen, P.J. Morin, M. a Thomsen, M.E. Power, C. Teplitsky, J.S. Alho, J. a Mills, J. Merila, J. Penuelas, Y. Willi, E. Johnson, T.G. Barraclough, J.S. Pal, R.J. Trapp, F. Giorgi, G.E. Hofmann, A. Maxmen, a H. Purcell, Sensing Chromosome BiOrientation Kinase from Kinetochore Substrates, Science. 323 (2009) 1350-1353. doi:10.1126/science.1167000

[149] G. Fang, H. Yu, M.W. Kirschner, Direct binding of CDC20 protein family members activates the anaphase-promoting complex in mitosis and G1., Mol. Cell. 2 (1998) 163-171. doi:10.1016/S10972765(00)80126-4.

[150] G. Fang, H. Yu, M.W. Kirschner, The checkpoint protein MAD2 and the mitotic regulator CDC20 form a ternary complex with the anaphase-promoting complex to control anaphase initiation, Genes Dev. 12 (1998) 1871-1883. doi:10.1101/gad.12.12.1871.

[151] S.H. Kim, D.P. Lin, S. Matsumoto, A. Kitazono, T. Matsumoto, Fission yeast Slp1: an effector of the Mad2-dependent spindle checkpoint., Science. 279 (1998) 1045-1047. doi:10.1126/science.279.5353.1045.

[152] L.H. Hwang, L.F. Lau, D.L. Smith, C.A. Mistrot, K.G. Hardwick, E.S. Hwang, A. Amon, A.W. Murray, Budding yeast Cdc20: a target of the spindle checkpoint., Science. 279 (1998) 1041-1044. doi:10.1126/science.279.5353.1041.

[153] O. Cohen-Fix, J.M.J.-M. Peters, M.W. Kirschner, D. Koshland, Anaphase initiation in Saccharomyces cerevisiae is controlled by the APC-dependent degradation of the anaphase inhibtitor Psd1p., Genes Dev. 10 (1996) 3081-3093. doi:10.1101/gad.10.24.3081.

[154] S. Irniger, S. Piatti, C. Michaelis, K. Nasmyth, Genes involved in sister chromatid separation are needed for b-type cyclin proteolysis in budding yeast, Cell. 81 (1995) 269-277. doi:10.1016/00928674(95)90337-2.

[155] R.W. King, J.M. Peters, S. Tugendreich, M. Rolfe, P. Hieter, M.W. Kirschner, A 20s complex containing CDC27 and CDC16 catalyzes the mitosis-specific conjugation of ubiquitin to cyclin B, Cell. 81 (1995) 279-288. doi:10.1016/0092-8674(95)90338-0.

[156] H. Zou, T.J. McGarry, T. Bernal, M.W. Kirschner, Identification of a vertebrate sister-chromatid separation inhibitor involved in transformation and tumorigenesis., Science. 285 (1999) 418-422. doi:10.1126/science.285.5426.418

[157] W.C.H. Chao, K. Kulkarni, Z. Zhang, E.H. Kong, D. Barford, Structure of the mitotic checkpoint complex, Nature. 484 (2012) 208-213. doi:10.1038/nature10896.

[158] V. Sudakin, G.K.T. Chan, T.J. Yen, Checkpoint inhibition of the APC/C in HeLa cells is mediated by a complex of BUBR1, BUB3, CDC20, and MAD2, J. Cell Biol. 154 (2001) 925-936. doi:10.1083/jcb.200102093.

[159] R. Fraschini, A. Beretta, L. Sironi, A. Musacchio, G. Lucchini, S. Piatti, Bub3 interaction with Mad2, Mad3 and Cdc20 is mediated by WD40 repeats and does not require intact kinetochores, EMBO J. 20 (2001) 6648-6659. doi:10.1093/emboj/20.23.6648.

[160] A.T. Saurin, M.S. van der Waal, R. eacute H. Medema, S.M.A. Lens, G.J.P.L. Kops, Aurora B potentiates Mps1 activation to ensure rapid checkpoint establishment at the onset of mitosis, Nat. Commun. 2 (2011) 316-319. doi:10.1038/ncomms1319.

[161] N. Jelluma, A.B. Brenkman, N.J.F. van den Broek, C.W.A. Cruijsen, M.H.J. van Osch, S.M.A. Lens, R.H. Medema, G.J.P.L. Kops, Mps1 Phosphorylates Borealin to Control Aurora B Activity and Chromosome Alignment, Cell. 132 (2008) 233-246. doi:10.1016/j.cell.2007.11.046.

[162] W. Nijenhuis, E. Von Castelmur, D. Littler, V. De Marco, E. Tromer, M. Vleugel, M.H.J. Van 
Osch, B. Snel, A. Perrakis, G.J.P.L. Kops, A TPR domain-containing N-terminal module of MPS1 is required for its kinetochore localization by Aurora B, J. Cell Biol. 201 (2013) 217-231. doi:10.1083/jcb.201210033.

[163] M. Vleugel, E. Tromer, M. Omerzu, V. Groenewold, W. Nijenhuis, B. Snel, G.J.P.L. Kops, Arrayed BUB recruitment modules in the kinetochore scaffold KNL1 promote accurate chromosome segregation., J. Cell Biol. 203 (2013) 943-955. doi:10.1083/jcb.201307016.

[164] Y. Yamagishi, C.-H. Yang, Y. Tanno, Y. Watanabe, MPS1/Mph1 phosphorylates the kinetochore protein KNL1/Spc7 to recruit SAC components, Nat. Cell Biol. 14 (2012) 746-752. doi:10.1038/ncb2515.

[165] I. Primorac, J.R. Weir, E. Chiroli, F. Gross, I. Hoffmann, S. van Gerwen, A. Ciliberto, A. Musacchio, Bub3 reads phosphorylated MELT repeats to promote spindle assembly checkpoint signaling, Elife. 2013 (2013) 1-20. doi:10.7554/eLife.01030.

[166] G. Zhang, T. Lischetti, J. Nilsson, A minimal number of MELT repeats supports all the functions of KNL1 in chromosome segregation., J. Cell Sci. 127 (2014) 871-84. doi:10.1242/jcs.139725.

[167] V. Krenn, K. Overlack, I. Primorac, S. Van Gerwen, A. Musacchio, KI motifs of human Knl1 enhance assembly of comprehensive spindle checkpoint complexes around MELT repeats, Curr. Biol. 24 (2014) 29-39. doi:10.1016/j.cub.2013.11.046.

[168] L.A. Shepperd, J.C. Meadows, A.M. Sochaj, T.C. Lancaster, J. Zou, G.J. Buttrick, J. Rappsilber, K.G. Hardwick, J.B.A. Millar, Phosphodependent recruitment of Bub1 and Bub3 to Spc7/KNL1 by Mph1 kinase maintains the spindle checkpoint, Curr. Biol. 22 (2012) 891-899. doi:10.1016/j.cub.2012.03.051.

[169] H. Sharp-Baker, R.H. Chen, Spindle checkpoint protein Bub1 is required for kinetochore localization of Mad1, Mad2, Bub3, and CENP-E, independently of its kinase activity, J. Cell Biol. 153 (2001) 1239-1249. doi:10.1083/jcb.153.6.1239.

[170] V.L. Johnson, M.I.F. Scott, S. V Holt, D. Hussein, S.S. Taylor, Bub1 is required for kinetochore localization of BubR1, Cenp-E, Cenp-F and Mad2, and chromosome congression., J. Cell Sci. 117 (2004) 1577-1589. doi:10.1242/jcs.01006.

[171] N. London, S. Biggins, Mad1 kinetochore recruitment by Mps1-mediated phosphorylation of Bub1 signals the spindle checkpoint, Genes Dev. 28 (2014) 140-152. doi:10.1101/gad.233700.113.

[172] M.S. van der Waal, A.T. Saurin, M.J.M. Vromans, M. Vleugel, C. Wurzenberger, D.W. Gerlich, R.H. Medema, G.J.P.L. Kops, S.M. a Lens, Mps1 promotes rapid centromere accumulation of Aurora B., EMBO Rep. 13 (2012) 847-54. doi:10.1038/embor.2012.93.

[173] G. Zhang, T. Lischetti, D.G. Hayward, J. Nilsson, Distinct domains in Bub1 localize RZZ and BubR1 to kinetochores to regulate the checkpoint., Nat. Commun. 6 (2015) 7162. doi:10.1038/ncomms8162.

[174] R. Karess, Rod-Zw10-Zwilch: A key player in the spindle checkpoint, Trends Cell Biol. 15 (2005) 386-392. doi:10.1016/j.tcb.2005.05.003.

[175] E. Buffin, C. Lefebvre, J. Huang, M.E. Gagou, R.E. Karess, Recruitment of Mad2 to the kinetochore requires the Rod/Zw10 complex, Curr. Biol. 15 (2005) 856-861. doi:10.1016/j.cub.2005.03.052.

[176] R. Basto, R. Gomes, R.E. Karess, Rough deal and Zw10 are required for the metaphase checkpoint in Drosophila., Nat. Cell Biol. 2 (2000) 939-943. doi:10.1038/35046592.

[177] M. Barisic, S. Geley, Spindly switch controls anaphase: Spindly and RZZ functions in chromosome attachment and mitotic checkpoint control, Cell Cycle. 10 (2011) 449-456. doi:10.4161/cc.10.3.14759.

[178] X. Luo, Z. Tang, G. Xia, K. Wassmann, T. Matsumoto, J. Rizo, H. Yu, The Mad2 spindle checkpoint protein has two distinct natively folded states., Nat. Struct. Mol. Biol. 11 (2004) 338345. doi:10.1038/nsmb748.

[179] X. Luo, G. Fang, M. Coldiron, Y. Lin, H. Yu, M.W. Kirschner, G. Wagner, Structure of the Mad2 spindle assembly check- point protein and its interaction with Cdc20, Nat. Struct. Biol. 7 (2000) 224-229. doi:doi:10.1038/73338. 
[180] L. Sironi, M. Mapelli, S. Knapp, A. De Antoni, K.T. Jeang, A. Musacchio, Crystal structure of the tetrameric Mad1-Mad2 core complex: Implications of a "safety belt" binding mechanism for the spindle checkpoint, EMBO J. 21 (2002) 2496-2506. doi:10.1093/emboj/21.10.2496.

[181] M. Mapelli, F. V Filipp, G. Rancati, L. Massimiliano, L. Nezi, G. Stier, R.S. Hagan, S. Confalonieri, S. Piatti, M. Sattler, A. Musacchio, Determinants of conformational dimerization of Mad2 and its inhibition by p31comet., EMBO J. 25 (2006) 1273-1284. doi:10.1038/sj.emboj.7601033.

[182] X. Luo, Z. Tang, J. Rizo, H. Yu, The Mad2 spindle checkpoint protein undergoes similar major conformational changes upon binding to either Mad1 or Cdc20, Mol. Cell. 9 (2002) 59-71. doi:10.1016/S1097-2765(01)00435-X.

[183] A. De Antoni, C.G. Pearson, D. Cimini, J.C. Canman, V. Sala, L. Nezi, M. Mapelli, L. Sironi, M. Faretta, E.D. Salmon, A. Musacchio, The Mad1/Mad2 complex as a template for Mad2 activation in the spindle assembly checkpoint, Curr. Biol. 15 (2005) 214-225. doi:10.1016/j.cub.2005.01.038.

[184] M. Vink, M. Simonetta, P. Transidico, K. Ferrari, M. Mapelli, A. De Antoni, L. Massimiliano, A. Ciliberto, M. Faretta, E.D. Salmon, A. Musacchio, In Vitro FRAP Identifies the Minimal Requirements for Mad2 Kinetochore Dynamics, Curr. Biol. 16 (2006) 755-766. doi:10.1016/j.cub.2006.03.057.

[185] E.R. Griffis, N. Stuurman, R.D. Vale, Spindly, a novel protein essential for silencing the spindle assembly checkpoint, recruits dynein to the kinetochore, J. Cell Biol. 177 (2007) 1005-1015. doi:10.1083/jcb.200702062.

[186] R. Gassmann, A.J. Holland, D. Varma, X. Wan, C. Filiz, K. Oegema, E.D. Salmon, A. Desai, Removal of Spindly from microtubule-attached kinetochores controls spindle checkpoint silencing in human cells, Genes Dev. 24 (2010) 957-971. doi:10.1101/gad.1886810.

[187] J.S. Rosenberg, F.R. Cross, H. Funabiki, KNL1/Spc105 recruits PP1 to silence the spindle assembly checkpoint, Curr. Biol. 21 (2011) 942-947. doi:10.1016/j.cub.2011.04.011.

[188] W. Nijenhuis, G. Vallardi, A. Teixeira, G.J.P.L. Kops, A.T. Saurin, Negative feedback at kinetochores underlies a responsive spindle checkpoint signal, Nature. 16 (2014) 1257-1264. doi:10.1038/ncb3065.

[189] S.A. Foster, D.O. Morgan, The APC/C Subunit Mnd2/Apc15 Promotes Cdc20 Autoubiquitination and Spindle Assembly Checkpoint Inactivation, Mol. Cell. 47 (2012) 921-932. doi:10.1016/j.molcel.2012.07.031.

[190] K. Uzunova, B.T. Dye, H. Schutz, R. Ladurner, G. Petzold, Y. Toyoda, M. a Jarvis, N.G. Brown, I. Poser, M. Novatchkova, K. Mechtler, A. a Hyman, H. Stark, B. a Schulman, J.-M. Peters, APC15 mediates CDC20 autoubiquitylation by APC/CMCC and disassembly of the mitotic checkpoint complex, Nat. Struct. Mol. Biol. 19 (2012) 1116-1123. doi:10.1038/nsmb.2412.

[191] J. Mansfeld, P. Collin, M.O. Collins, J.S. Choudhary, J. Pines, APC15 drives the turnover of MCCCDC20 to make the spindle assembly checkpoint responsive to kinetochore attachment, Nat. Cell Biol. 13 (2011) 1234-1243. doi:10.1038/ncb2347.

[192] X. Li, R.B. Nicklas, Mitotic forces control a cell-cycle checkpoint., Nature. 373 (1995) 630-2. doi:10.1038/373630a0.

[193] R.B. Nicklas, J.C. Waters, E.D. Salmon, S.C. Ward, Checkpoint signals in grasshopper meiosis are sensitive to microtubule attachment, but tension is still essential., J. Cell Sci. 114 (2001) 41734183.

[194] B. Etemad, T.E.F. Kuijt, G.J.P.L. Kops, Kinetochore-microtubule attachment is sufficient to satisfy the human spindle assembly checkpoint., Nat. Commun. 6 (2015) 8987. doi:10.1038/ncomms9987.

[195] E.C. Tauchman, F.J. Boehm, J.G. DeLuca, Stable kinetochore-microtubule attachment is sufficient to silence the spindle assembly checkpoint in human cells, Nat. Commun. 6 (2015) 8987. doi:10.1038/ncomms9987.

[196] P. Aravamudhan, A.A. Goldfarb, A.P. Joglekar, The kinetochore encodes a mechanical switch to disrupt spindle assembly checkpoint signalling, Nat. Cell Biol. 17 (2015) 868-879. doi:10.1038/ncb3179.

[197] B.J. Howell, B. Moree, E.M. Farrar, S. Stewart, G. Fang, E.D. Salmon, Spindle checkpoint protein 
dynamics at kinetochores in living cells, Curr. Biol. 14 (2004) 953-964.

doi:10.1016/j.cub.2004.05.053.

[198] D. Liu, M. Vleugel, C.B. Backer, T. Hori, T. Fukagawa, I.M. Cheeseman, M.A. Lampson, Regulated targeting of protein phosphatase 1 to the outer kinetochore by KNL1 opposes Aurora B kinase, J. Cell Biol. 188 (2010) 809-820. doi:10.1083/jcb.201001006.

[199] J. Maciejowski, K.A. George, M.E. Terret, C. Zhang, K.M. Shokat, P. V. Jallepalli, Mps1 directs the assembly of Cdc20 inhibitory complexes during interphase and mitosis to control $\mathrm{M}$ phase timing and spindle checkpoint signaling, J. Cell Biol. 190 (2010) 89-100. doi:10.1083/jcb.201001050.

[200] S. Santaguida, A. Tighe, A.M. D'Alise, S.S. Taylor, A. Musacchio, Dissecting the role of MPS1 in chromosome biorientation and the spindle checkpoint through the small molecule inhibitor reversine, J. Cell Biol. 190 (2010) 73-87. doi:10.1083/jcb.201001036.

[201] L. Hewitt, A. Tighe, S. Santaguida, A.M. White, C.D. Jones, A. Musacchio, S. Green, S.S. Taylor, Sustained Mps1 activity is required in mitosis to recruit O-Mad2 to the Mad1-C-Mad2 core complex, J. Cell Biol. 190 (2010) 25-34. doi:10.1083/jcb.201002133.

[202] J.C. Meadows, L.A. Shepperd, V. Vanoosthuyse, T.C. Lancaster, A.M. Sochaj, G.J. Buttrick, K.G. Hardwick, J.B.A. Millar, Spindle checkpoint silencing requires association of PP1 to both Spc7 and kinesin-8 motors, Dev. Cell. 20 (2011) 739-750. doi:10.1016/j.devcel.2011.05.008.

[203] J.Y. Hsu, Z.W. Sun, X. Li, M. Reuben, K. Tatchell, D.K. Bishop, J.M. Grushcow, C.J. Brame, J.A. Caldwell, D.F. Hunt, R. Lin, M.M. Smith, C.D. Allis, Mitotic phosphorylation of histone H3 is governed by Ipl1/aurora kinase and Glc7/PP1 phosphatase in budding yeast and nematodes., Cell. 102 (2000) 279-291. doi:10.1016/S0092-8674(00)00034-9.

[204] B.A. Pinsky, C.R. Nelson, S. Biggins, Protein Phosphatase 1 Regulates Exit from the Spindle Checkpoint in Budding Yeast, Curr. Biol. 19 (2009) 1182-1187. doi:10.1016/j.cub.2009.06.043.

[205] V. Vanoosthuyse, K.G. Hardwick, A Novel Protein Phosphatase 1-Dependent Spindle Checkpoint Silencing Mechanism, Curr. Biol. 19 (2009) 1176-1181. doi:10.1016/j.cub.2009.05.060.

[206] B. a Pinsky, C. V Kotwaliwale, Y. Sean, S.Y. Tatsutani, C. a Breed, S. Biggins, Glc7 / Protein Phosphatase 1 Regulatory Subunits Can Oppose the Ipl1 / Aurora Protein Kinase by Redistributing Glc7, Mol. Cell. Biol. 26 (2006) 2648-2660. doi:10.1128/MCB.26.7.2648.

[207] L. Francisco, W. Wang, C.S. Chan, Type 1 protein phosphatase acts in opposition to IpL1 protein kinase in regulating yeast chromosome segregation., Mol. Cell. Biol. 14 (1994) 4731-40. doi:10.1128/MCB.14.7.4731.Updated.

[208] S.J.E.J.E. Suijkerbuijk, M. Vleugel, A. Teixeira, G.J.P.L.J.P.L. Kops, Integration of Kinase and Phosphatase Activities by BUBR1 Ensures Formation of Stable Kinetochore-Microtubule Attachments, Dev. Cell. 23 (2012) 745-755. doi:10.1016/j.devcel.2012.09.005.

[209] K. Overlack, I. Primorac, M. Vleugel, V. Krenn, S. Maffini, I. Hoffmann, G.J.P.L. Kops, A. Musacchio, A molecular basis for the differential roles of Bub1 and BubR1 in the spindle assembly checkpoint., Elife. 4 (2015) 1-24. doi:10.7554/eLife.05269.

[210] T. Kruse, G. Zhang, M.S.Y. Larsen, T. Lischetti, W. Streicher, T. Kragh Nielsen, S.P. Bjørn, J. Nilsson, Direct binding between BubR1 and B56-PP2A phosphatase complexes regulate mitotic progression., J. Cell Sci. 126 (2013) 1086-92. doi:10.1242/jcs.122481.

[211] P. Xu, E. a Raetz, M. Kitagawa, D.M. Virshup, S.H. Lee, BUBR1 recruits PP2A via the B56 family of targeting subunits to promote chromosome congression., Biol. Open. 2 (2013) 479-86. doi:10.1242/bio.20134051.

[212] A. Espert, P. Uluocak, R.N. Bastos, D. Mangat, P. Graab, U. Gruneberg, PP2A-B56 opposes Mps1 phosphorylation of Knl1 and thereby promotes spindle assembly checkpoint silencing, J. Cell Biol. 206 (2014) 833-842. doi:10.1083/jcb.201406109.

[213] T.U. Tanaka, M.J.R. Stark, K. Tanaka, Kinetochore capture and bi-orientation on the mitotic spindle, Nat. Rev. Mol. Cell Biol. 6 (2005) 929-942. doi:10.1038/nrm1764.

[214] H. Maiato, J. DeLuca, E.D. Salmon, W.C. Earnshaw, The dynamic kinetochore-microtubule interface, J. Cell Sci. 117 (2004) 5461-5477. doi:10.1242/jcs.01536. 
[215] J.. R. McIntosh, E.L. Grishchuk, R.R. West, Chromosome-Microtubule Interactions During Mitosis, Annu. Rev. Cell Dev. Biol. 18 (2002) 193-219. doi:10.1146/annurev.cellbio.18.032002.132412.

[216] C.L. Rieder, E.D. Salmon, The vertebrate cell kinetochore and its roles during mitosis, Trends Cell Biol. 8 (1998) 310-318. doi:10.1016/S0962-8924(98)01299-9.

[217] Y.-T. Lin, Y. Chen, G. Wu, W.-H. Lee, Hec1 sequentially recruits Zwint-1 and ZW10 to kinetochores for faithful chromosome segregation and spindle checkpoint control., Oncogene. 25 (2006) 6901-14. doi:10.1038/sj.onc.1209687.

[218] J.G. DeLuca, B.J. Howell, J.C. Canman, J.M. Hickey, G. Fang, E.D. Salmon, Nuf2 and Hec1 Are Required for Retention of the Checkpoint Proteins Mad1 and Mad2 to Kinetochores, 2003. doi:10.1016/j.cub.2003.10.056.

[219] R. Bharadwaj, W. Qi, H. Yu, Identification of Two Novel Components of the Human NDC80 Kinetochore Complex, J. Biol. Chem. 279 (2004) 13076-13085. doi:10.1074/jbc.M310224200.

[220] S. Sivakumar, P.Ł. Janczyk, Q. Qu, C.A. Brautigam, P.T. Stukenberg, H. Yu, G.J. Gorbsky, The human Ska complex drives the metaphase-anaphase cell cycle transition by recruiting protein phosphatase 1 to kinetochores., Elife. 5 (2016). doi:10.7554/eLife.12902.

[221] A.K. Dunsch, E. Linnane, F.A. Barr, U. Gruneberg, The astrin-kinastrin/SKAP complex localizes to microtubule plus ends and facilitates chromosome alignment, J. Cell Biol. 192 (2011) 959-968. doi:10.1083/jcb.201008023.

[222] C. Ditchfield, V.L. Johnson, A. Tighe, R. Ellston, C. Haworth, T. Johnson, A. Mortlock, N. Keen, S.S. Taylor, Aurora B couples chromosome alignment with anaphase by targeting BubR1, Mad2, and Cenp-E to kinetochores, J. Cell Biol. 161 (2003) 267-280. doi:10.1083/jcb.200208091.

[223] O. Söderberg, M. Gullberg, M. Jarvius, K. Ridderstråle, K.-J. Leuchowius, J. Jarvius, K. Wester, P. Hydbring, F. Bahram, L.-G. Larsson, U. Landegren, Direct observation of individual endogenous protein complexes in situ by proximity ligation., Nat. Methods. 3 (2006) 995-1000. doi:10.1038/nmeth947.

[224] M.S. Kelker, R. Page, W. Peti, Crystal Structures of Protein Phosphatase-1 Bound to Nodularin-R and Tautomycin: A Novel Scaffold for Structure-based Drug Design of Serine/Threonine Phosphatase Inhibitors, J. Mol. Biol. 385 (2009) 11-21. doi:10.1016/j.jmb.2008.10.053.

[225] J.R. Kremer, D.N. Mastronarde, J.R. McIntosh, Computer Visualization of Three-Dimensional Image Data Using IMOD, J. Struct. Biol. 116 (1996) 71-76. doi:10.1006/jsbi.1996.0013.

[226] B. Banerjee, C.A. Kestner, P.T. Stukenberg, EB1 enables spindle microtubules to regulate centromeric recruitment of Aurora B, J. Cell Biol. 204 (2014) 947-963. doi:10.1083/jcb.201307119.

[227] S. Sivakumar, G.J. Gorbsky, Spatiotemporal regulation of the anaphase-promoting complex in mitosis., Nat. Rev. Mol. Cell Biol. 16 (2015) 82-94. doi:10.1038/nrm3934.

[228] D. Stevens, R. Gassmann, K. Oegema, A. Desai, Uncoordinated loss of chromatid cohesion is a common outcome of extended metaphase arrest, PLoS One. 6 (2011). doi:10.1371/journal.pone.0022969.

[229] E. a. Foley, M. Maldonado, T.M. Kapoor, Formation of stable attachments between kinetochores and microtubules depends on the B56-PP2A phosphatase, Nat. Cell Biol. 13 (2011) 1265-1271. doi:10.1038/ncb2327.

[230] E. Heroes, B. Lesage, J. Görnemann, M. Beullens, L. Van Meervelt, M. Bollen, The PP1 binding code: A molecular-lego strategy that governs specificity, FEBS J. 280 (2013) 584-595. doi:10.1111/j.1742-4658.2012.08547.x.

[231] W. Peti, A.C. Nairn, R. Page, Structural basis for protein phosphatase 1 regulation and specificity, FEBS J. 280 (2013) 596-611. doi:10.1111/j.1742-4658.2012.08509.x.

[232] A.N. Godet, J. Guergnon, V. Maire, A. Croset, A. Garcia, The combinatorial PP1-binding consensus motif $(\mathrm{R} / \mathrm{K}) \mathrm{x}(0,1) \mathrm{v} / \mathrm{IxFxx}(\mathrm{R} / \mathrm{K}) \mathrm{x}(\mathrm{R} / \mathrm{K})$ is a new apoptotic signature, PLoS One. 5 (2010) 1-8. doi:10.1371/journal.pone.0009981.

[233] T. Hollin, C. De Witte, A. Lenne, C. Pierrot, J. Khalife, Analysis of the interactome of the Ser/Thr 
Protein Phosphatase type 1 in Plasmodium falciparum, BMC Genomics. 17 (2016) 246. doi:10.1186/s12864-016-2571-z.

[234] A. Garcia, X. Cayla, J. Guergnon, F. Dessauge, V. Hospital, M.P. Rebollo, A. Fleischer, A. Rebollo, Serine/threonine protein phosphatases PP1 and PP2A are key players in apoptosis, Biochimie. 85 (2003) 721-726. doi:10.1016/j.biochi.2003.09.004.

[235] J. Qian, E. Vafiadaki, S.M. Florea, V.P. Singh, W. Song, C.K. Lam, Y. Wang, Q. Yuan, T.J. Pritchard, W. Cai, K. Haghighi, P. Rodriguez, H.S. Wang, D. Sanoudou, G.C. Fan, E.G. Kranias, Small heat shock protein 20 interacts with protein phosphatase-1 and enhances sarcoplasmic reticulum calcium cycling, Circ. Res. 108 (2011) 1429-1438. doi:10.1161/CIRCRESAHA.110.237644.

[236] N. London, S. Ceto, J.A. Ranish, S. Biggins, Phosphoregulation of Spc105 by Mps1 and PP1 regulates Bub1 localization to kinetochores, Curr. Biol. 22 (2012) 900-906. doi:10.1016/j.cub.2012.03.052.

[237] A. Hirschi, M. Cecchini, R.C. Steinhardt, M.R. Schamber, F. a Dick, S.M. Rubin, An overlapping kinase and phosphatase docking site regulates activity of the retinoblastoma protein., Nat. Struct. Mol. Biol. 17 (2010) 1051-1057. doi:10.1038/nsmb.1868.

[238] M.H. Schmitz, M. Held, V. Janssens, J.R. Hutchins, O. Hudecz, E. Ivanova, J. Goris, L. TrinkleMulcahy, A.I. Lamond, I. Poser, A. a Hyman, K. Mechtler, J.-M. Peters, D.W. Gerlich, Live-cell imaging RNAi screen identifies PP2A-B55alpha and importin-beta1 as key mitotic exit regulators in human cells., Nat. Cell Biol. 12 (2010) 886-893. doi:10.1038/ncb2092.

[239] R. Della Monica, R. Visconti, N. Cervone, A.F. Serpico, D. Grieco, Fep1 phosphatase controls Greatwall kinase to promote PP2A-B55 activation and mitotic progression., Elife. 4 (2015) e10399. doi:10.7554/eLife.10399.

[240] A. Grallert, E. Boke, A. Hagting, B. Hodgson, Y. Connolly, J.R. Griffiths, D.L. Smith, J. Pines, I.M. Hagan, A PP1-PP2A phosphatase relay controls mitotic progression., Nature. 517 (2015) 948. doi:10.1038/nature14019.

[241] S. Mochida, S. Ikeo, J. Gannon, T. Hunt, Regulated activity of PP2A-B55 delta is crucial for controlling entry into and exit from mitosis in Xenopus egg extracts., EMBO J. 28 (2009) 27772785. doi:10.1038/emboj.2009.238.

[242] T. Glatter, A. Wepf, R. Aebersold, M. Gstaiger, An integrated workflow for charting the human interaction proteome: insights into the PP2A system., Mol. Syst. Biol. 5 (2009) 237. doi:10.1038/msb.2008.75.

[243] T. Zhu, Z. Dou, B. Qin, C. Jin, X. Wang, L. Xu, Z. Wang, L. Zhu, F. Liu, X. Gao, Y. Ke, Z. Wang, F. Aikhionbare, C. Fu, X. Ding, X. Yao, Phosphorylation of microtubule-binding protein hec1 by mitotic kinase aurora $\mathrm{b}$ specifies spindle checkpoint kinase mps1 signaling at the kinetochore, J. Biol. Chem. 288 (2013) 36149-36159. doi:10.1074/jbc.M113.507970.

[244] J. Espeut, D.K. Cheerambathur, L. Krenning, K. Oegema, A. Desai, Microtubule binding by KNL1 contributes to spindle checkpoint silencing at the kinetochore, J. Cell Biol. 196 (2012) 469-482. doi:10.1083/jcb.201111107.

[245] Y. Kim, A.J. Holland, W. Lan, D.W. Cleveland, Aurora Kinases and Protein Phosphatase 1 Mediate Chromosome Congression through Regulation of CENP-E, Cell. 142 (2010) 444-455. doi:10.1016/j.cell.2010.06.039.

[246] M. Posch, G.A. Khoudoli, S. Swift, E.M. King, J.G. DeLuca, J.R. Swedlow, Sds22 regulates aurora B activity and microtubule-kinetochore interactions at mitosis, J. Cell Biol. 191 (2010) 61-74. doi:10.1083/jcb.200912046.

[247] L. Trinkle-Mulcahy, J. Andersen, W.L. Yun, G. Moorhead, M. Mann, A.I. Lamond, Repo-Man recruits PP1 $\gamma$ to chromatin and is essential for cell viability, J. Cell Biol. 172 (2006) 679-692. doi: $10.1083 /$ jcb. 200508154 .

[248] A. Eiteneuer, J. Seiler, M. Weith, M. Beullens, B. Lesage, V. Krenn, A. Musacchio, M. Bollen, H. Meyer, Inhibitor-3 ensures bipolar mitotic spindle attachment by limiting association of SDS22 with kinetochore-bound protein phosphatase-1., EMBO J. 33 (2014) 2704-2720. doi:10.15252/embj.201489054. 
[249] C. Wurzenberger, M. Held, M.A. Lampson, I. Poser, A.A. Hyman, D.W. Gerlich, Sds22 and repoman stabilize chromosome segregation by counteracting Aurora B on anaphase kinetochores, J. Cell Biol. 198 (2012) 173-183. doi:10.1083/jcb.201112112.

[250] J. Qian, M. Beullens, B. Lesage, M. Bollen, Aurora B defines its own chromosomal targeting by opposing the recruitment of the phosphatase scaffold Repo-Man, Curr. Biol. 23 (2013) 1136-1143. doi:10.1016/j.cub.2013.05.017.

[251] J. Qian, B. Lesage, M. Beullens, A. Van Eynde, M. Bollen, PP1/repo-man dephosphorylates mitotic histone H3 at T3 and regulates chromosomal aurora B targeting, Curr. Biol. 21 (2011) 766-773. doi:10.1016/j.cub.2011.03.047.

[252] I.M. Porter, K. Schleicher, M. Porter, J.R. Swedlow, Bod1 regulates protein phosphatase 2A at mitotic kinetochores., Nat. Commun. 4 (2013) 2677. doi:10.1038/ncomms3677.

[253] P. Xu, D.M. Virshup, S.H. Lee, B56-PP2A regulates motor dynamics for mitotic chromosome alignment., J. Cell Sci. 127 (2014) 4567-73. doi:10.1242/jcs.154609.

[254] T.H. Scheuermann, S.B. Padrick, K.H. Gardner, C.A. Brautigam, On the acquisition and analysis of microscale thermophoresis data, Anal. Biochem. 496 (2015) 79-93. doi:10.1016/j.ab.2015.12.013.

[255] T. Kanda, K.F. Sullivan, G.M. Wahl, Histone-GFP fusion protein enables sensitive analysis of chromosome dynamics in living mammalian cells., Curr. Biol. 8 (1998) 377-385. doi:10.1016/S0960-9822(98)70156-3.

[256] Z. Tang, R. Bharadwaj, B. Li, H. Yu, Mad2-Independent Inhibition of APCCdc20 by the Mitotic Checkpoint Protein BubR1, Dev. Cell. 1 (2001) 227-237. doi:10.1016/S1534-5807(01)00019-3.

[257] C.A. Brautigam, Calculations and Publication-Quality Illustrations for Analytical Ultracentrifugation Data, in: Methods Enzymol., 2015: pp. 109-133.

doi:10.1016/bs.mie.2015.05.001. 